\title{
Numerical Evidence for the Equivariant Birch and Swinnerton-Dyer Conjecture
}

\author{
Werner Bley
}

\section{CONTENTS}

1. Introduction

2. Algebraic Preliminaries

3. The Equivariant Tamagawa Number Conjecture for the Base Change of an Elliptic Curve

4. Explicit Version of the Equivariant Tamagawa Number Conjecture

5. Computational Remarks

6. Examples

Acknowledgments

References

2000 AMS Subject Classification: 11G40, 14G10, 11G05

Keywords: Birch-Swinnerton-Dyer conjecture, Equivariant Tamagawa Number conjecture
Let $E / \mathbb{Q}$ be an elliptic curve and $K / \mathbb{Q}$ a finite Galois extension with group $G$. We write $E_{K}$ for the base change of $E$ and consider the equivariant Tamagawa number conjecture for the pair $\left(h^{1}\left(E_{K}\right)(1), \mathbb{Z}[G]\right)$. This conjecture is an equivariant refinement of the Birch and Swinnerton-Dyer conjecture for $E / K$. For almost all primes I, we derive an explicit formulation of the conjecture that makes it amenable to numerical verifications. We use this to provide convincing numerical evidence in favor of the conjecture.

\section{INTRODUCTION}

Let $E / \mathbb{Q}$ be an elliptic curve and let $K / \mathbb{Q}$ be a finite Galois extension with group $G=\operatorname{Gal}(K / \mathbb{Q})$. We write $E_{K}$ for the base change of $E$. We consider the motive $M=h^{1}(E)(1)$ and regard

$$
M_{K}:=h^{0}(\operatorname{Spec}(K)) \otimes_{h^{0}(\operatorname{Spec}(\mathbb{Q}))} M=h^{1}\left(E_{K}\right)(1)
$$

as a motive over $\mathbb{Q}$ with a natural left action of the rational group ring $\mathbb{Q}[G]$ via the first factor. We write $\zeta(\mathbb{C}[G])$ for the center of the complex group ring $\mathbb{C}[G]$, and $L\left(M_{K}, s\right)$ for the $\zeta(\mathbb{C}[G])$-valued $L$-function of $M_{K}$, which is defined and analytic in $\operatorname{Re}(s)>1 / 2$. It is conjectured that $L\left(M_{K}, s\right)$ has a meromorphic continuation to all of $\mathbb{C}$. Assuming this conjecture, we write $L^{*}\left(M_{K}\right)$ for the leading term in its Taylor expansion at $s=0$. To be more explicit, we let $\operatorname{Irr}(G)$ be the set of absolutely irreducible characters of $G$. For any character $\chi$ we write $L(E / \mathbb{Q}, \chi, s)$ for the twisted Hasse-Weil $L$-function, and $L^{*}(E / \mathbb{Q}, \chi, 1)$ for the leading term in the Taylor expansion at $s=1$. The center $\zeta(\mathbb{C}[G])$ is canonically isomorphic to $\prod_{\chi \in \operatorname{Irr}(G)} \mathbb{C}$, and via this identification, $L^{*}\left(M_{K}\right)$ equals $\left(L^{*}(E / \mathbb{Q}, \bar{\chi}, 1)\right)_{\chi \in \operatorname{Irr}(G)}$. It is easily shown that $L^{*}\left(M_{K}\right) \in \zeta(\mathbb{R}[G])^{\times}$(see Remark 3.2).

The equivariant Tamagawa number conjecture (ETNC, for short) formulated in [Burns and Flach 01] for the pair $\left(M_{K}, \mathbb{Z}[G]\right)$ is equivalent to an equality of the form

$$
\delta\left(L^{*}\left(M_{K}\right)\right)=\chi\left(M_{K}\right)
$$


where $\delta$ is a canonical homomorphism from the unit group $\zeta(\mathbb{R}[G])^{\times}$to the relative algebraic $K$-group $K_{0}(\mathbb{Z}[G], \mathbb{R})$, and $\chi\left(M_{K}\right)$ is a certain Euler characteristic in this relative group constructed from the various motivic cohomology groups, realizations, comparison isomorphisms, and regulators associated to $M_{K}$ and its Kummer dual.

We note in passing that the ETNC is formulated in much more generality and that the general formulation is comparatively abstract. Indeed, in our elliptic curve case with $K=\mathbb{Q}$, the ETNC is equivalent to the Birch and Swinnerton-Dyer conjecture (BSD for short). But even this basic fact is not evident, and we refer the reader to [Kings 09] or [Venjakob 07] for a detailed proof.

For the base change of an elliptic curve, the ETNC is an "equivariant BSD conjecture." Our main result, Proposition 4.5, makes this apparent for arbitrary elliptic curves $E / \mathbb{Q}$ and Galois extensions $K / \mathbb{Q}$.

The aim of this article is to describe an approach for converting the rather involved and abstract conjectural equality (1-1) into a form that is amenable to numerical computations. In this way, we systematically improve upon work in [Navilarekallu 88], which originally was the motivation for this manuscript.

As in the classical case of the BSD conjecture, the ETNC splits into three parts: an "equivariant rank conjecture," an "equivariant rationality conjecture," and an "equivariant integrality conjecture."

We will use [Dokchitser 04] to compute numerical approximations to the leading terms of the twisted HasseWeil $L$-functions, and for our general approach we will then usually assume the validity of the rank conjecture. However, in our concrete examples in Section 6, we are often able to deduce the rank conjecture from theoretical results or from an explicit computation of Selmer groups.

We then show how to compute numerical approximations to equivariant periods and to equivariant regulators (provided that we are able to compute the MordellWeil group $E(K)$ ). Combining these computations, we are able to verify the rationality conjecture numerically up to the precision of our computations.

From now on, we assume the validity of the equivariant rationality conjecture. We note in passing that there are important results in the literature (without being exhaustive, we mention only [Gross and Zagier 86, Kolyvagin 90, Kolyvagin and Logachev 90, Kolyvagin and Logachev 92, Zhang 01] and recent results of Bertolini and Darmon) from which one can possibly deduce the equivariant rationality conjecture provided that the analytic (equivariant) rank is at most 1 . This will be part of a further research project. In our numerical examples we consider mostly elliptic curves $E$ defined over $\mathbb{Q}$ and dihedral extensions $K / \mathbb{Q}$ of order $2 l$ for an odd prime $l$ such that the Mordell-Weil group $E(K)$ is finite. In this case, where all absolutely irreducible characters are of degree 1 or 2, the important works [Shimura 77, Shimura 78] probably allow one to deduce the equivariant rationality conjecture. In a slightly different situation, namely for subextensions of the false Tate curve tower, in [Bouganis and Dokchitser 07] the authors successfully apply Shimura's work to deduce algebraicity and Galois equivariance of twisted BSD quotients. Similar arguments will hopefully work in our context.

Furthermore, we assume throughout that the TateShafarevich group $\amalg(E / K)$ is finite. Again, it is possible to deduce the finiteness of $\amalg(E / K)$ in many examples, provided that the analytic rank is at most 1 , from the above-mentioned work. However, since the aim of this paper is to work out the additional difficulties of the equivariant conjecture, we prefer to make the general assumption that $\amalg(E / K)$ is finite.

A further main result of this paper (Corollary 4.8) shows that we can use the computational results from the verification of the rationality conjecture to prove the $l$-part of the ETNC for all primes $l$ outside a finite set of difficult primes. This finite set contains in most cases the prime divisors of $\# G$ and the prime divisors of $\# \amalg(E / K)$.

There are two different reasons that we get into difficulties with these primes. Our approach is restricted to the case that certain cohomology groups are perfect $\mathbb{Z}_{l}[G]$-modules. If $l \nmid \# G$, the ring $\mathbb{Z}_{l}[G]$ is regular, so that this assumption is satisfied for every finitely generated $\mathbb{Z}_{l}[G]$-module. On the other hand, even if $l \mid \# G$, there are some rare cases in which the modules under consideration are perfect, so that we can also produce numerical evidence for these interesting primes (see the examples in Section 6).

Primes dividing $\# \amalg(E / K)$ are difficult just because we are not able to compute $\amalg(E / K)$ as a Galois module (which would be necessary in order to compute Euler characteristics). The situation is even worse, because we do not have an algorithm at our disposal to compute $\# \amalg(E / K)$. In order to compute a conjectural candidate for the set of difficult primes, we will assume the validity of the classical BSD conjecture for $E / K$ and use it to compute a conjectural value for $\# \amalg(E / K)$. 
We point out that the equivariant BSD conjecture has far-reaching explicit consequences (see [Burns 09]) that could not be derived from nonequivariant versions. This may indicate that any theoretical or numerical verification of the equivariant version requires a great deal of additional effort. Much of the additional algorithmic problems are hidden in the algorithms described in [Bley and Wilson 09]. In particular, it can be shown that the ETNC is true if and only if the twisted BSD quotients satisfy certain congruences. For cyclic groups $Z_{p}$, dihedral groups $D_{2 p}$ with $p$ an odd prime, and the alternating group $A_{4}$ we explicitly determine these congruences.

We will illustrate our results in Section 6 with some explicit examples. More examples can be computed using the author's Magma implementations. ${ }^{1}$

The structure of the paper is as follows. In the first part of Section 2, we review algebraic preliminaries such as determinant functors, categories of virtual objects, and the construction of Euler characteristics in these categories. These very abstract concepts are used to formulate the ETNC in [Burns and Flach 01]. Following an approach in [Burns 04], we then make the construction of Euler characteristics more explicit in terms of relative algebraic $K$-groups and in this way amenable to numerical computations. In particular, in the second part of Section 2, we recall the algorithmic methods of [Bley and Wilson 09], which allow the computation of the relevant relative algebraic $K$-groups and provide methods to compute in them. For certain small groups we explicitly determine the above-mentioned congruences.

In Section 3 we describe the ETNC for the base change of an elliptic curve, and in Section 4 we then derive our main theoretical results (Proposition 4.5 and Corollary 4.8 ), which are the basis for our numerical computations. In Section 5 we comment on the algorithmic aspects of our work, and in the final section we describe several interesting examples in detail. In particular, we have chosen the examples such that we can apply our methods for a prime divisor $l$ of $\# G$ and such that the explicit congruences can be seen.

In future work [Bley 10] we will study the $l$-part of ETNC for elliptic curves $E / K$ and cyclic extensions $K / \mathbb{Q}$ of prime-power order $l^{n}$, for $l$ odd.

\footnotetext{
${ }^{1}$ Available from http://www.mathematik.uni-muenchen.de/ bley/
} pub.html.

\section{ALGEBRAIC PRELIMINARIES}

\subsection{Determinant Functors and Virtual Objects}

Let $R$ by any associative unital ring. Let $\operatorname{PMod}(R)$ denote the category of finitely generated projective $R$-modules, and write $\operatorname{PMod}(R)^{\bullet}$ for the category of bounded complexes of such modules. We also write $D(R)$ for the derived category of complexes of $R$-modules and $D^{\text {perf }}(R)$ for the full triangulated subcategory of $D(R)$ consisting of those complexes that are isomorphic in $D(R)$ to an object in $\operatorname{PMod}(R)^{\bullet}$. These complexes are called perfect. Recall that $C^{\bullet}$ is perfect if and only if there exist a complex $P^{\bullet} \in \operatorname{PMod}(R)^{\bullet}$ and a quasiisomorphism $P^{\bullet} \longrightarrow C^{\bullet}$. We say that an $R$-module $N$ is perfect if the complex $N[0]$ belongs to $D^{\text {perf }}(R)$.

Our main reference for determinant functors, Picard categories, and virtual objects is [Burns and Flach 01]. Let $V(R)$ denote the Picard category of virtual objects associated to $\operatorname{PMod}(R)$ and write $[\cdot]_{R}$ for the universal determinant functor

$$
[\cdot]_{R}:(\operatorname{PMod}(R), \text { is }) \longrightarrow V(R),
$$

where $(\operatorname{PMod}(R)$, is) denotes the subcategory of all isomorphisms in $\operatorname{PMod}(R)$. By [Burns and Flach 01, Proposition 2.1], this functor extends to a functor

$$
[\cdot]_{R}:\left(D^{\text {perf }}(R), \text { is }\right) \longrightarrow V(R) .
$$

We recall that $V(R)$ is equipped with a canonical bifunctor $(L, M) \mapsto L M$. We fix a unit object $\mathbf{1}_{R}$ and for each object $L$, an inverse $L^{-1}$ with an isomorphism $L L^{-1} \simeq$ $\mathbf{1}_{R}$. Each element of $V(R)$ is of the form $[P]_{R}[Q]_{R}^{-1}$ for modules $P, Q \in \operatorname{PMod}(R)$. Furthermore, $[P]_{R}$ and $[Q]_{R}$ are isomorphic in $V(R)$ if and only if their classes in $K_{0}(R)$ coincide.

For any Picard category $\mathcal{P}$ we define $\pi_{0}(\mathcal{P})$ to be the group of isomorphism classes of objects of $\mathcal{P}$ and set $\pi_{1}(\mathcal{P}):=\operatorname{Aut}_{\mathcal{P}}\left(\mathbf{1}_{\mathcal{P}}\right)$. The groups $\pi_{0}(V(R))$ and $\pi_{1}(V(R))$ are naturally isomorphic to $K_{0}(R)$ and $K_{1}(R)$, respectively.

Let $A$ be a finite-dimensional semisimple $\mathbb{Q}$-algebra. For any extension field $F$ of $\mathbb{Q}$ we put $A_{F}:=A \otimes_{\mathbb{Q}} F$ and abbreviate $A_{p}:=A \otimes_{\mathbb{Q}} \mathbb{Q}_{p}$. Let $\mathcal{A} \subseteq A$ be a $\mathbb{Z}$-order and set

$$
\begin{aligned}
\mathcal{A}_{p} & :=\mathcal{A} \otimes_{\mathbb{Z}} \mathbb{Z}_{p}, \quad \hat{\mathcal{A}}:=\mathcal{A} \otimes_{\mathbb{Z}} \hat{\mathbb{Z}} \simeq \prod_{p} \mathcal{A}_{p}, \\
\hat{A} & :=A \otimes_{\mathbb{Z}} \hat{\mathbb{Z}} \simeq \prod_{p} A_{p} .
\end{aligned}
$$

For $L \in \operatorname{PMod}(\mathcal{A})$ we set

$$
L_{p}:=L \otimes_{\mathbb{Z}} \mathbb{Z}_{p}, \quad L_{\mathbb{Q}}:=L \otimes_{\mathbb{Z}} \mathbb{Q}, \quad L_{F}:=L \otimes_{\mathbb{Z}} F .
$$


We set $\mathbb{V}(\mathcal{A}):=V(\hat{\mathcal{A}}) \times_{V(\hat{A})} V(A)$ and recall that elements in $\mathbb{V}(\mathcal{A})$ are of the form $(\hat{X}, Y, \hat{\theta})$ with $\hat{X} \in$ $V(\hat{\mathcal{A}}), Y \in V(A)$ and $\hat{\theta}: \hat{X} \otimes_{\hat{\mathcal{A}}} \hat{A} \stackrel{\simeq}{\longrightarrow} Y \otimes_{A} \hat{A}$ an isomorphism in $V(\hat{A})$. Note that the tensor in this context is the functor between categories of virtual objects induced by the tensor functor on the level of modules by the universal property [Burns and Flach 01, (2.3),f]. Note also that we can identify $\hat{X}$ with $\prod_{p} X_{p} \in \prod_{p} V\left(\mathcal{A}_{p}\right)$, where $X_{p}:=\hat{X} \otimes_{\hat{\mathcal{A}}} \mathcal{A}_{p} \in V\left(\mathcal{A}_{p}\right)$.

There is a natural monoidal functor $V(\mathcal{A}) \longrightarrow \mathbb{V}(\mathcal{A})$ induced by

$$
[L]_{\mathcal{A}} \mapsto\left(\prod_{p}\left[L_{p}\right]_{\mathcal{A}_{p}},\left[L_{\mathbb{Q}}\right]_{A}, \prod_{p}\left[\theta_{p}\right]\right),
$$

where

$$
\theta_{p}: L_{p} \otimes_{\mathbb{Z}_{p}} \mathbb{Q}_{p}=L \otimes_{\mathbb{Z}} \mathbb{Q}_{p} \stackrel{\mathrm{id}}{\longrightarrow} L \otimes_{\mathbb{Z}} \mathbb{Q}_{p}=L_{\mathbb{Q}} \otimes_{\mathbb{Q}} \mathbb{Q}_{p}
$$

is the natural map.

Let $\mathcal{P}_{0}$ be the Picard category with unique object $\mathbf{1}_{\mathcal{P}_{0}}$ and $\operatorname{Aut}_{\mathcal{P}_{0}}\left(\mathbf{1}_{\mathcal{P}_{0}}\right)=0$. Following [Burns and Flach 01], we define $\mathbb{V}(\mathcal{A}, F)$ to be the fiber product category $\mathbb{V}(\mathcal{A}) \times_{V\left(A_{F}\right)} \mathcal{P}_{0}$. Explicitly, elements in $\mathbb{V}(\mathcal{A}, F)$ are given by triples

$$
\left((\hat{X}, Y, \hat{\theta}), \mathbf{1}_{\mathcal{P}_{0}}, \theta_{\infty}\right),
$$

where

$$
\begin{gathered}
\hat{X} \in V(\hat{\mathcal{A}}), \quad Y \in V(A), \\
\hat{\theta}: \hat{X} \otimes_{\hat{\mathcal{A}}} \hat{A} \stackrel{\simeq}{\longrightarrow} Y \otimes_{A} \hat{A}, \\
\theta_{\infty}: Y \otimes_{A} A_{F} \stackrel{\simeq}{\longrightarrow} \mathbf{1}_{A_{F}} .
\end{gathered}
$$

We usually omit $\mathbf{1}_{\mathcal{P}_{0}}$ in the notation.

By [Burns and Flach 01, Proposition 2.5], one has a canonical isomorphism

$$
\iota_{\mathcal{A}, F}: \pi_{0} \vee(\mathcal{A}, F) \simeq K_{0}(\mathcal{A}, F),
$$

where $K_{0}(\mathcal{A}, F)$ is the relative algebraic $K$-group as defined in [Swan 68, p. 215]. Following the proof of [Burns and Flach 01, Proposition 2.5], we explicitly describe the inverse of $\iota_{\mathcal{A}, F}$. Let $[P, \varphi, Q]$ be an element in $K_{0}(\mathcal{A}, F)$ with $P, Q \in \operatorname{PMod}(\mathcal{A})$ and an isomorphism $\varphi: P \otimes_{\mathcal{A}} A_{F} \longrightarrow Q \otimes_{\mathcal{A}} A_{F}$ of $A_{F}$-modules. Then

$$
\begin{aligned}
\iota_{\mathcal{A}, F}^{-1} & ([P, \varphi, Q]) \\
= & \left(\left(\prod_{p}\left[P_{p}\right]_{\mathcal{A}_{p}}\left[Q_{p}\right]_{\mathcal{A}_{p}}^{-1},\left[P \otimes_{\mathcal{A}} A\right]_{A}\left[Q \otimes_{\mathcal{A}} A\right]_{A}^{-1}, \prod_{p} \theta_{p}\right),\right. \\
& \left.\varphi_{\text {triv }}\right),
\end{aligned}
$$

where each $\theta_{p}$ is the canonical map and $\varphi_{\text {triv }}$ is the composite

$$
\begin{aligned}
& {\left[P \otimes_{\mathcal{A}} A_{F}\right]_{A_{F}}\left[Q \otimes_{\mathcal{A}} A_{F}\right]_{A_{F}}^{-1}} \\
& \quad \longrightarrow\left[Q \otimes_{\mathcal{A}} A_{F}\right]_{A_{F}}\left[Q \otimes_{\mathcal{A}} A_{F}\right]_{A_{F}}^{-1} \longrightarrow \mathbf{1}_{A_{F}} .
\end{aligned}
$$

Here the first isomorphism is induced by $\varphi$.

If we set $V(\mathcal{A}, F):=V(\mathcal{A}) \times_{V\left(A_{F}\right)} \mathcal{P}_{0}$, then the functor $V(\mathcal{A}) \longrightarrow \mathbb{V}(\mathcal{A})$ induces a canonical functor $V(\mathcal{A}, F) \longrightarrow \mathbb{V}(\mathcal{A}, F)$ and a homomorphism

$$
\pi_{0} V(\mathcal{A}, F) \longrightarrow \pi_{0} \vee(\mathcal{A}, F),
$$

and hence a homomorphism $\pi_{0} V(\mathcal{A}, F) \longrightarrow K_{0}(\mathcal{A}, F)$, which we also denote by $\iota_{\mathcal{A}, F}$. In the same way, we obtain isomorphisms (see again [Burns and Flach 01, Proposition 2.5])

$$
\iota_{\mathcal{A}_{p}, \mathbb{Q}_{p}}: \pi_{0} V\left(\mathcal{A}_{p}, \mathbb{Q}_{p}\right) \simeq K_{0}\left(\mathcal{A}_{p}, \mathbb{Q}_{p}\right) .
$$

Given data as in (2-1), we therefore obtain an element

$$
\iota_{\mathcal{A}, F}\left(\left((\hat{X}, Y, \hat{\theta}), \theta_{\infty}\right)\right) \in K_{0}(\mathcal{A}, F) .
$$

In the context of the ETNC, we are in addition given an element $\mathcal{L}^{*} \in \zeta\left(A_{F}\right)^{\times}$. There is a canonical commutative diagram of the form shown in Figure 1.

If $R$ is a finite-dimensional semisimple algebra over either a global field or a local field, then we have an injective reduced norm map

$$
\operatorname{Nrd}_{R}: K_{1}(R) \longrightarrow \zeta(R)^{\times} .
$$

If $G$ is a finite group, then $\operatorname{Nrd}_{\mathbb{R}[G]}$ is in general not surjective. However, by [Burns and Flach 01, Section 4.2], there always exists a canonical "extended boundary homomorphism"

$$
\delta: \zeta(\mathbb{R}[G])^{\times} \longrightarrow K_{0}(\mathbb{Z}[G], \mathbb{R})
$$

such that $\quad \delta \circ \operatorname{Nrd}_{\mathbb{R}[G]}=\partial_{\mathbb{Z}[G], \mathbb{R}}^{1}$. See [Breuning and Burns 07, Section 2.1.2] for a conceptual description of $\delta$.

The conjectures we wish to consider in this paper are essentially of the form

$$
T \Omega:=\iota_{\mathbb{Z}[G], \mathbb{R}}\left(\left((\hat{X}, Y, \hat{\theta}), \theta_{\infty}\right)\right)-\delta\left(\mathcal{L}^{*}\right)=0
$$

in $K_{0}(\mathbb{Z}[G], \mathbb{R})$. In the sequel we set $\mathcal{A}=\mathbb{Z}[G], A=$ $\mathbb{Q}[G]$, and $F=\mathbb{R}$.

For our computational purposes, in particular to be able to apply the results and algorithms of [Bley and Wilson 09], we need to reinterpret this construction in terms of explicit elements of $K_{0}(\mathcal{A}, F)$. Here we essentially follow the approach in [Burns 04]. For any bounded complex $P^{\bullet}$ we define objects $P^{\text {all }}, P^{\text {even }}$, and 


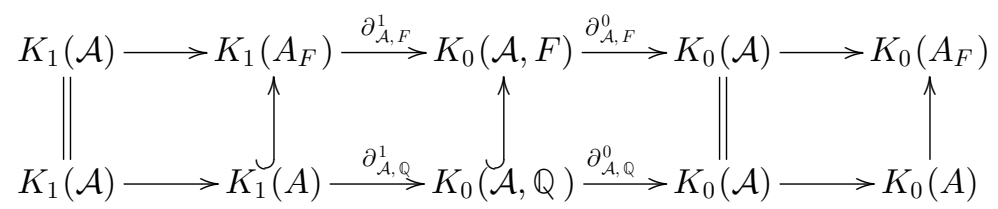

FIGURE 1. Localization sequences.

$P^{\text {odd }}$ by

$$
P^{\text {all }}=\bigoplus_{i \in \mathbb{Z}} P^{i}, \quad P^{\text {even }}=\bigoplus_{i \text { even }} P^{i}, \quad P^{\text {odd }}=\bigoplus_{i \text { odd }} P^{i} .
$$

We write $Z^{\bullet}\left(P^{\bullet}\right), B^{\bullet}\left(P^{\bullet}\right)$, and $H^{\bullet}\left(P^{\bullet}\right)$ for the complexes of cycles, boundaries, and cohomology of $P^{\bullet}$, each with zero differentials.

In arithmetic applications we often have the following data:

\section{Data 2.1.}

(a) $Y^{\text {even }}, Y^{\text {odd }} \in \operatorname{PMod}(A)$, together with an $A_{F^{-}}$ isomorphism

$$
Y^{\text {even }} \otimes_{\mathbb{Q}} F \stackrel{\theta_{F}}{\longrightarrow} Y^{\text {odd }} \otimes_{\mathbb{Q}} F
$$

(b) $X_{p}^{\bullet} \in \operatorname{PMod}\left(\mathcal{A}_{p}\right)^{\bullet}$ together with isomorphisms

$$
\begin{aligned}
& H^{\text {even }}\left(X_{p}^{\bullet}\right) \otimes_{\mathbb{Z}_{p}} \mathbb{Q}_{p} \stackrel{\theta_{p}^{\text {even }}}{\longrightarrow} Y^{\text {even }} \otimes_{\mathbb{Q}} \mathbb{Q}_{p}, \\
& H^{\text {odd }}\left(X_{p}^{\bullet}\right) \otimes_{\mathbb{Z}_{p}} \mathbb{Q}_{p} \stackrel{\theta_{p}^{\text {odd }}}{\longrightarrow} Y^{\text {odd }} \otimes_{\mathbb{Q}} \mathbb{Q}_{p} .
\end{aligned}
$$

(c) $\mathcal{L}^{*} \in \zeta\left(A_{F}\right)^{\times}$.

These data are related to the data given in (2-1) in the following way:

$$
\begin{aligned}
\hat{X} & =\prod_{p}\left[X_{p}^{\bullet}\right]_{\mathcal{A}_{p}}, \\
Y & =\left[Y^{\text {even }}\right]_{A}\left[Y^{\text {odd }}\right]_{A}^{-1}, \\
\theta_{\infty}: Y \otimes_{A} A_{F} & =\left[Y^{\text {even }} \otimes_{A} A_{F}\right]_{A_{F}}\left[Y^{\text {odd }} \otimes_{A} A_{F}\right]_{A_{F}}^{-1} \\
\simeq & {\left[Y^{\text {odd }} \otimes_{A} A_{F}\right]_{A_{F}}\left[Y^{\text {odd }} \otimes_{A} A_{F}\right]_{A_{F}}^{-1} } \\
\simeq & \mathbf{1}_{A_{F}}, \text { with the first isomorphism } \\
& \text { induced by } \theta_{F}, \\
\theta_{p}: X_{p} & =\left[X_{p}^{\bullet} \otimes_{\mathbb{Z}_{p}} \mathbb{Q}_{p}\right]_{A_{p}} \\
& \stackrel{\alpha_{1}}{\simeq}\left[H^{\text {even }}\left(X_{p}^{\bullet} \otimes_{\mathbb{Z}_{p}} \mathbb{Q}_{p}\right)\right]_{A_{p}} \\
& \times\left[H^{\text {odd }}\left(X_{p}^{\bullet} \otimes_{\mathbb{Z}_{p}} \mathbb{Q}_{p}\right)\right]_{A_{p}}^{-1} \\
& \left.\left.\stackrel{\alpha_{2}}{\simeq}\left[Y^{\text {even }} \otimes_{\mathbb{Q}} \mathbb{Q}_{p}\right)\right]_{A_{p}}\left[Y^{\text {odd }} \otimes_{\mathbb{Q}} \mathbb{Q}_{p}\right)\right]_{A_{p}}^{-1} \\
= & {\left[Y \otimes_{\mathbb{Q}} \mathbb{Q}_{p}\right]_{A_{p}} . }
\end{aligned}
$$

Here $\alpha_{1}$ is the canonical isomorphism of [Burns and Flach 01, Proposition 2.1e] and $\alpha_{2}$ is induced by $\theta_{p}^{\text {even }}$ and $\theta_{p}^{\text {odd }}$.

Let $\mathbb{C}_{p}$ denote the completion of a fixed algebraic closure of $\mathbb{Q}_{p}$. For every prime $p$ and every homomorphism $j: \mathbb{R} \rightarrow \mathbb{C}_{p}$ we obtain induced maps $j_{*}: K_{0}(\mathbb{Z}[G], \mathbb{R}) \rightarrow$ $K_{0}\left(\mathbb{Z}_{p}[G], \mathbb{C}_{p}\right)$ and $j_{*}: \zeta(\mathbb{R}[G])^{\times} \longrightarrow \zeta\left(\mathbb{C}_{p}[G]\right)^{\times}$. We fix $p$ and $j$ and consider the map $\theta_{p}^{\prime}: X_{p}^{\text {even }} \otimes_{\mathbb{Z}_{p}} \mathbb{C}_{p} \longrightarrow$ $X_{p}^{\text {odd }} \otimes_{\mathbb{Z}_{p}} \mathbb{C}_{p}$ defined by

$$
\begin{aligned}
& X_{p}^{\text {even }} \otimes_{\mathbb{Z}_{p}} \mathbb{C}_{p} \\
& \quad \stackrel{\beta_{1}}{\simeq}\left(H^{\text {even }}\left(X_{p}^{\bullet}\right) \otimes_{\mathbb{Z}_{p}} \mathbb{C}_{p}\right) \oplus\left(B^{\text {all }}\left(X_{p}^{\bullet}\right) \otimes_{\mathbb{Z}_{p}} \mathbb{C}_{p}\right) \\
& \quad \stackrel{\beta_{2}}{\simeq}\left(Y^{\text {even }} \otimes_{\mathbb{Z}_{p}} \mathbb{C}_{p}\right) \oplus\left(B^{\text {all }}\left(X_{p}^{\bullet}\right) \otimes_{\mathbb{Z}_{p}} \mathbb{C}_{p}\right) \\
& \quad \stackrel{\beta_{3}}{\simeq}\left(Y^{\text {odd }} \otimes_{\mathbb{Z}_{p}} \mathbb{C}_{p}\right) \oplus\left(B^{\text {all }}\left(X_{p}^{\bullet}\right) \otimes_{\mathbb{Z}_{p}} \mathbb{C}_{p}\right) \\
& \quad \stackrel{\beta_{4}}{\simeq}\left(H^{\text {odd }}\left(X_{p}^{\bullet}\right) \otimes_{\mathbb{Z}_{p}} \mathbb{C}_{p}\right) \oplus\left(B^{\text {all }}\left(X_{p}^{\bullet}\right) \otimes_{\mathbb{Z}_{p}} \mathbb{C}_{p}\right) \\
& \quad \stackrel{\beta_{5}}{\simeq} X_{p}^{\text {odd }} \otimes_{\mathbb{Z}_{p}} \mathbb{C}_{p} .
\end{aligned}
$$

The isomorphisms $\beta_{1}$ and $\beta_{5}$ are induced by choosing splittings of the tautological exact sequences

$$
\begin{aligned}
0 \longrightarrow & Z^{i}\left(X_{p}^{\bullet} \otimes_{\mathbb{Q}_{p}} \mathbb{C}_{p}\right) \longrightarrow X_{p}^{i} \otimes_{\mathbb{Q}_{p}} \mathbb{C}_{p} \\
& \longrightarrow B^{i+1}\left(X_{p}^{\bullet} \otimes_{\mathbb{Q}_{p}} \mathbb{C}_{p}\right) \longrightarrow 0, \\
0 \longrightarrow B^{i}\left(X_{p}^{\bullet} \otimes_{\mathbb{Q}_{p}} \mathbb{C}_{p}\right) \longrightarrow Z^{i}\left(X_{p}^{\bullet} \otimes_{\mathbb{Q}_{p}} \mathbb{C}_{p}\right) & \longrightarrow 0,
\end{aligned}
$$

while $\beta_{2}$ and $\beta_{4}$ are induced by $\theta_{p}^{\text {even }}$ and $\theta_{p}^{\text {odd }}$, respectively, and $\beta_{3}$ by $\theta_{F}$. It can be shown that the refined Euler characteristic $\left[X_{p}^{\text {even }}, \theta_{p}^{\prime}, X_{p}^{\text {odd }}\right] \in K_{0}\left(\mathbb{Z}_{p}[G], \mathbb{C}_{p}\right)$ does not depend on any of the above choices. See [Breuning and Burns 05, Section 6] or [Burns 04] for more information on this construction of refined Euler characteristics and its relation to the Euler characteristic used in [Burns and Flach 01].

Lemma 2.2. Assume that $X_{p}^{\bullet} \otimes_{\mathbb{Z}_{p}} \mathbb{C}_{p}$ is acyclic outside degrees 1 and 2 . Then

$$
j_{*}\left(\iota_{\mathbb{Z}[G], \mathbb{R}}\left(\left(\hat{X}, Y, \prod_{p} \theta_{p}\right), \theta_{\infty}\right)\right)=\left[X_{p}^{\text {even }}, \theta_{p}^{\prime}, X_{p}^{\text {odd }}\right] .
$$


Proof. We consider the diagram

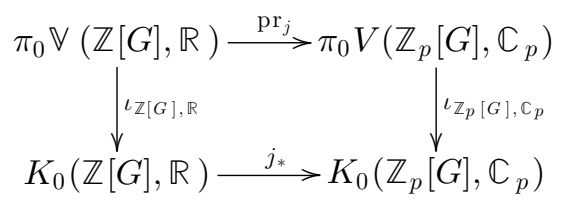

It follows from the explicit descriptions of $\iota_{\mathbb{Z}[G], \mathbb{R}}^{-1}$ and $\iota_{\mathbb{Z}_{p}[G], \mathbb{C}_{p}}^{-1}$ that the diagram is commutative. From [Breuning and Burns 05, Theorem 6.2 and Corollary 6.3], we deduce that

$\iota_{\mathbb{Z}_{p}[G], \mathbb{C}_{p}}\left(\operatorname{pr}_{j}\left(\left(\hat{X}, Y, \prod_{p} \theta_{p}\right), \theta_{\infty}\right)\right)=\left[X_{p}^{\text {even }}, \theta_{p}^{\prime}, X_{p}^{\text {odd }}\right]$.

Hence the result follows.

Remark 2.3. Although [Breuning and Burns 05, Theorem 6.2] is more general, we chose to formulate Lemma 2.2 with the acyclicity condition. This simplification suffices for our applications, and moreover, we can use the additivity result [Breuning and Burns 05, Theorem 5.7] without introducing any correction terms.

Let $C$ denote a finite perfect $\mathbb{Z}_{p}[G]$-module. Recall that a finite $\mathbb{Z}_{p}[G]$-module $C$ is perfect if and only if there exists a projective resolution

$$
0 \longrightarrow P^{-1} \stackrel{\alpha}{\longrightarrow} P^{0} \longrightarrow C \longrightarrow 0
$$

of length 2. Then the element

$$
\chi_{\mathbb{Z}_{p}[G], \mathbb{Q}_{p}}(C):=\left[P^{-1}, \alpha \otimes \mathbb{Q}_{p}, P^{0}\right] \in K_{0}\left(\mathbb{Z}_{p}[G], \mathbb{Q}_{p}\right)
$$

does not depend on the choice of the above resolution.

For any $\mathbb{Z}_{p}[G]$-module $P$ we write $P_{\text {tors }}$ for the submodule of $\mathbb{Z}_{p}$-torsion elements and set $P_{\mathrm{tf}}:=P / P_{\mathrm{tors}}$.

Lemma 2.4. Assume that we have data given as in Data 2.1. Let $p$ be a fixed prime and $j: \mathbb{R} \longrightarrow \mathbb{C}_{p}$ an embedding.

(a) If all cohomology modules $H^{i}\left(X_{p}^{\bullet}\right), i \in \mathbb{Z}$, are $\mathbb{Z}_{p}[G]$ perfect, then

$$
\begin{gathered}
j_{*}\left(\iota_{\mathbb{Z}[G], \mathbb{R}}\left(\left(\hat{X}, Y, \prod_{p} \theta_{p}\right), \theta_{\infty}\right)\right) \\
=\left[H^{\text {even }}\left(X_{p}^{\bullet}\right), \theta_{p}^{\prime \prime}, H^{\text {odd }}\left(X_{p}^{\bullet}\right)\right]
\end{gathered}
$$

with $\theta_{p}^{\prime \prime}=\left(\theta_{p}^{\text {odd }} \otimes \mathbb{C}_{p}\right)^{-1} \circ\left(\theta_{F} \otimes \mathbb{C}_{p}\right) \circ\left(\theta_{p}^{\text {even }} \otimes \mathbb{C}_{p}\right)$. (b) If, in addition, $H^{i}\left(X_{p}^{\bullet}\right)_{\text {tors }}$ is $\mathbb{Z}_{p}[G]$-perfect for all $i \in$ $\mathbb{Z}$, then

$$
\begin{gathered}
j_{*}\left(\iota_{\mathbb{Z}[G], \mathbb{R}}\left(\left(\hat{X}, Y, \prod_{p} \theta_{p}\right), \theta_{\infty}\right)\right) \\
=\left[H^{\text {even }}\left(X_{p}^{\bullet}\right)_{\mathrm{tf}}, \theta_{p}^{\prime \prime}, H^{\text {odd }}\left(X_{p}^{\bullet}\right)_{\mathrm{tf}}\right] \\
-\chi_{\mathbb{Z}_{p}[G], \mathbb{Q}_{p}}\left(H^{\text {even }}\left(X_{p}^{\bullet}\right)_{\text {tors }}\right) \\
\quad+\chi_{\mathbb{Z}_{p}[G], \mathbb{Q}_{p}}\left(H^{\text {odd }}\left(X_{p}^{\bullet}\right)_{\text {tors }}\right) .
\end{gathered}
$$

Proof. Part (a) follows from the definition of $\theta_{p}^{\prime}$ and [Burns and Flach 01, Proposition 2.1e]. If the modules $H^{\text {even }}\left(X_{p}^{\bullet}\right)_{\text {tors }}$ and $H^{\text {odd }}\left(X_{p}^{\bullet}\right)_{\text {tors }}$ are also $\mathbb{Z}_{p}[G]$-perfect, then

$$
\begin{aligned}
j_{*} & \left(\iota_{\mathbb{Z}[G], \mathbb{R}}\left(\left(\hat{X}, Y, \prod_{p} \theta_{p}\right), \theta_{\infty}\right)\right) \\
= & {\left[H^{\text {even }}\left(X_{p}^{\bullet}\right)_{\mathrm{tf}}, \theta_{p}^{\prime \prime}, H^{\text {odd }}\left(X_{p}^{\bullet}\right)_{\mathrm{tf}}\right] } \\
& +\left[H^{\text {even }}\left(X_{p}^{\bullet}\right)_{\text {tors }}, 0, H^{\text {odd }}\left(X_{p}^{\bullet}\right)_{\text {tors }}\right] .
\end{aligned}
$$

Part (b) follows now from the definition of the second summand by dévissage.

For later reference, we record the following lemma. We write $\delta_{p}: \zeta\left(\mathbb{C}_{p}[G]\right)^{\times} \longrightarrow K_{0}\left(\mathbb{Z}_{p}[G], \mathbb{C}_{p}\right)$ for the extended boundary homomorphism. Note that

$$
\delta_{p}=\partial_{\mathbb{Z}_{p}[G], \mathbb{C}_{p}}^{1} \circ \operatorname{Nrd}_{\mathbb{C}_{p}[G]}^{-1} \quad \text { and } \quad \delta_{p} \circ j_{*}=j_{*} \circ \delta .
$$

\section{Lemma 2.5.}

(a) Let $\xi \in \zeta(\mathbb{R}[G])^{\times}$. Then

$$
\delta(\xi) \in K_{0}(\mathbb{Z}[G], \mathbb{Q}) \Longleftrightarrow \xi \in \zeta(\mathbb{Q}[G])^{\times} .
$$

(b) Let $\xi \in \zeta\left(\mathbb{C}_{p}[G]\right)^{\times}$. Then

$$
\delta_{p}(\xi) \in K_{0}\left(\mathbb{Z}_{p}[G], \mathbb{Q}_{p}\right) \Longleftrightarrow \xi \in \zeta\left(\mathbb{Q}_{p}[G]\right)^{\times} .
$$

Proof. We recall the definition of $\delta$. By the weak approximation theorem, we may choose $\lambda \in \zeta(\mathbb{Q}[G])^{\times}$such that $\lambda \xi$ is in the image of the reduced norm map $\operatorname{Nrd}_{\mathbb{R}[G]}$. We shorten $\partial_{\mathbb{Z}[G], \mathbb{R}}^{1}$ to $\partial^{1}$. Then

$$
\delta(\xi)=\partial^{1}\left(\operatorname{Nrd}_{\mathbb{R}[G]}^{-1}(\lambda \xi)\right)-\sum_{p} \delta_{p}(\lambda),
$$

and therefore

$$
\delta(\xi) \in K_{0}(\mathbb{Z}[G], \mathbb{Q}) \Longleftrightarrow \partial^{1}\left(\operatorname{Nrd}_{\mathbb{R}[G]}^{-1}(\lambda \xi)\right) \in K_{0}(\mathbb{Z}[G], \mathbb{Q}) .
$$

An easy diagram chase using Figure 1 implies that $\partial^{1}\left(\operatorname{Nrd}_{\mathbb{R}[G]}^{-1}(\lambda \xi)\right) \in K_{0}(\mathbb{Z}[G], \mathbb{Q})$ if and only if there 
exists $\eta \in K_{1}(\mathbb{Q}[G])$ such that

$$
\operatorname{Nrd}_{\mathbb{R}[G]}^{-1}(\lambda \xi) / \eta \in \operatorname{im}\left(K_{1}(\mathbb{Z}[G]) \longrightarrow K_{1}(\mathbb{Q}[G])\right) .
$$

It follows that $\xi \in \zeta(\mathbb{Q}[G])$.

Let $\delta_{\mathbb{Q}}: \zeta(\mathbb{Q}[G])^{\times} \longrightarrow K_{0}(\mathbb{Z}[G], \mathbb{Q})$. The reverse implication is immediate from the commutative diagram

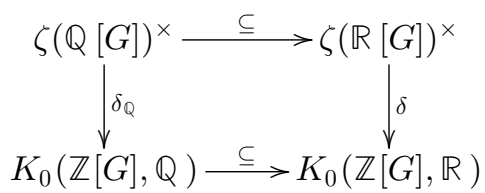

The proof of (b) is similar, but easier, because in the local case the reduced norm map is an isomorphism.

Proposition 2.6. Assume the situation of Data 2.1. Let $p$ be a prime and $j: \mathbb{R} \longrightarrow \mathbb{C}_{p}$ an embedding. Assume that $X_{p}^{\text {even }}$ and $X_{p}^{\text {odd }}$ are free $\mathbb{Z}_{p}[G]$-modules. Let $\left(v_{1}, \ldots, v_{d}\right)$ and $\left(w_{1}, \ldots, w_{d}\right)$ denote $\mathbb{Q}_{p}[G]$-basis of $X_{p}^{\text {even }} \otimes_{\mathbb{Z}_{p}} \mathbb{Q}_{p}$ and $X_{p}^{\text {odd }} \otimes_{\mathbb{Z}_{p}} \mathbb{Q}_{p}$, respectively. Let $B \in \mathrm{Gl}_{d}\left(\mathbb{C}_{p}[G]\right)$ represent $\theta_{p}^{\prime}$ with respect to the chosen basis. Set

$$
\Omega_{p}:=\left[X_{p}^{\text {even }}, \theta_{p}^{\prime}, X_{p}^{\text {odd }}\right]-j_{*}\left(\delta\left(\mathcal{L}^{*}\right)\right) .
$$

Then

$$
\begin{aligned}
\Omega_{p} & \in K_{0}\left(\mathbb{Z}_{p}[G], \mathbb{Q}_{p}\right) \\
& \Longleftrightarrow \operatorname{Nrd}_{\mathbb{C}_{p}[G]}(B) / j_{*}\left(\mathcal{L}^{*}\right) \in \zeta\left(\mathbb{Q}_{p}[G]\right)^{\times} .
\end{aligned}
$$

Proof. Let $F^{0}:=\mathbb{Z}_{p}[G] v_{1} \oplus \cdots \oplus \mathbb{Z}_{p}[G] v_{d}$ and $F^{1}:=$ $\mathbb{Z}_{p}[G] w_{1} \oplus \cdots \oplus \mathbb{Z}_{p}[G] w_{d}$. Then

$$
\left[F^{0}, B, F^{1}\right]-\left[X_{p}^{\text {even }}, \theta_{p}^{\prime}, X_{p}^{\text {odd }}\right] \in K_{0}\left(\mathbb{Z}_{p}[G], \mathbb{Q}_{p}\right) .
$$

Writing $\partial_{p}^{1}=\partial_{\mathbb{Z}_{p}[G], \mathbb{C}_{p}}^{1}$, one therefore has

$$
\begin{aligned}
\Omega_{p} & \in K_{0}\left(\mathbb{Z}_{p}[G], \mathbb{Q}_{p}\right) \\
& \Longleftrightarrow\left[F^{0}, B, F^{1}\right]-j_{*}\left(\delta\left(\mathcal{L}^{*}\right)\right) \in K_{0}\left(\mathbb{Z}_{p}[G], \mathbb{Q}_{p}\right) \\
& \Longleftrightarrow \partial_{p}^{1}\left(\left[\mathbb{C}_{p}[G]^{r}, B\right]\right)-\delta_{p}\left(j_{*}\left(\mathcal{L}^{*}\right)\right) \in K_{0}\left(\mathbb{Z}_{p}[G], \mathbb{Q}_{p}\right) \\
& \Longleftrightarrow \delta_{p}\left(\operatorname{Nrd}_{\mathbb{C}_{p}[G]}(B) / j_{*}\left(\mathcal{L}^{*}\right)\right) \in K_{0}\left(\mathbb{Z}_{p}[G], \mathbb{Q}_{p}\right) \\
& \Longleftrightarrow \operatorname{Nrd}_{\mathbb{C}_{p}[G]}(B) / j_{*}\left(\mathcal{L}^{*}\right) \in \zeta\left(\mathbb{Q}_{p}[G]\right)^{\times},
\end{aligned}
$$

where the last equivalence follows from Lemma 2.5.

In the next section we will recall from [Bley and Wilson 09] how the relative algebraic $K$ group $K_{0}\left(\mathbb{Z}_{p}[G], \mathbb{Q}_{p}\right)$ can be explicitly computed as an abstract abelian group and how the element $\Omega_{p}$ can be realized as an element of this abstract group.

Remark 2.7. (a) The assumption in Proposition 2.6 is no restriction because we can always find a projective $\mathbb{Z}_{p}[G]$-module $Z$ such that both $X_{p}^{\text {even }} \oplus Z$ and $X_{p}^{\text {odd }} \oplus Z$ are $\mathbb{Z}_{p}[G]$-free. Indeed, the canonical map $K_{0}\left(\mathbb{Z}_{p}[G]\right) \longrightarrow K_{0}\left(\mathbb{C}_{p}[G]\right)$ is injective by
[Curtis and Reiner 87, (32.1)], so that the existence of the isomorphism $\theta_{p}^{\prime}$ implies that $\left[X_{p}^{\text {even }}\right]=\left[X_{p}^{\text {odd }}\right]$ in $K_{0}\left(\mathbb{Z}_{p}[G]\right)$. By [Curtis and Reiner 87, II, p. 79], we further conclude that $X_{p}^{\text {even }} \simeq X_{p}^{\text {odd }}$ as $\mathbb{Z}_{p}[G]$-modules.

(b) The definition of $\theta_{p}^{\prime}$ shows that for rationality questions it is enough to consider the map

$$
Y^{\text {even }} \otimes_{\mathbb{Z}} \mathbb{R} \longrightarrow Y^{\text {odd }} \otimes_{\mathbb{Z}} \mathbb{R}
$$

induced by $\theta_{\mathbb{R}}$. More explicitly, let $\mathbb{Q}[G]=A_{1} \oplus \cdots \oplus A_{r}$ be the Wedderburn decomposition of $\mathbb{Q}[G]$ with corresponding idempotents $e_{1}, \ldots, e_{r}$. We set $Y_{i}^{\text {even }}:=e_{i} Y^{\text {even }}$ and $Y_{i}^{\text {odd }}:=e_{i} Y^{\text {odd }}$. Then each $A_{i}$ is a central simple algebra, and we denote by $S_{i}$ the unique simple $A_{i}$-module. Then $Y_{i}^{\text {even }} \simeq S_{i}^{d_{i}}$ and $Y_{i}^{\text {odd }} \simeq S_{i}^{d_{i}}$. By abuse of language, we refer to the explicit choice of such isomorphisms as a "choice of $\mathbb{Q}[G]$-bases" for $Y^{\text {even }}$ and $Y^{\text {odd }}$.

These isomorphisms combine with $\theta_{\mathbb{R}}$ to define an isomorphism

$$
\begin{aligned}
\tau: \bigoplus_{i=1}^{r}\left(S_{i}^{d_{i}} \otimes_{\mathbb{Q}} \mathbb{R}\right) & \simeq Y^{\text {even }} \otimes_{\mathbb{Q}} \mathbb{R} \stackrel{\theta_{\mathbb{R}}}{\longrightarrow} Y^{\text {odd }} \otimes_{\mathbb{Q}} \mathbb{R} \\
& \simeq \bigoplus_{i=1}^{r}\left(S_{i}^{d_{i}} \otimes_{\mathbb{Q}} \mathbb{R}\right) .
\end{aligned}
$$

Then one has

$$
\Omega \in K_{0}(\mathbb{Z}[G], \mathbb{Q}) \Longleftrightarrow \operatorname{Nrd}_{\mathbb{R}[G]}(\tau) / \mathcal{L}^{*} \in \zeta(\mathbb{Q}[G])^{\times} .
$$

\subsection{Relative algebraic $K$-groups}

In this section we recall results from [Bley and Wilson 09] that will be used to perform explicit computations in the relative algebraic $K$-groups $K_{0}(\mathbb{Z}[G], \mathbb{Q})$ and $K_{0}\left(\mathbb{Z}_{p}[G], \mathbb{Q}_{p}\right)$. For the definitions of these groups we refer the reader to [Swan 68, p. 215] or [Bley and Wilson 09, Section 2.1].

We set $\mathcal{A}:=\mathbb{Z}[G], A:=\mathbb{Q}[G]$, and choose a maximal order $\mathcal{M}$ in $A$ containing $\mathcal{A}$. We take $C:=\zeta(A)$ to be the center of $A$ and write $\mathcal{O}_{C}$ for the maximal order in $C$.

We let $D T\left(\mathcal{A}_{p}\right)$ denote the torsion subgroup of the finitely generated abelian group $K_{0}\left(\mathcal{A}_{p}, \mathbb{Q}_{p}\right)$. It is well known that the map on relative groups induced by the functor $\mathcal{M}_{p} \otimes_{\mathcal{A}_{p}}$ - gives the top exact sequence of the commutative diagram displayed in Figure 2. The vertical maps are induced by the reduced norm map (see [Bley and Wilson 09, Theorem 2.2 and 2.4] for more details).

For $\xi \in K_{0}\left(\mathcal{A}_{p}, \mathbb{Q}_{p}\right)$ we will often write $\tilde{\xi}$ for any lift of $\xi$ via the middle vertical isomorphism, i.e., $\delta_{p}(\tilde{\xi})=\xi$.

Next we briefly recall from [Bley and Wilson 09, Section 2.2] how the diagram in Figure 2 can be used to perform explicit computations in $K_{0}\left(\mathcal{A}_{p}, \mathbb{Q}_{p}\right)$. 


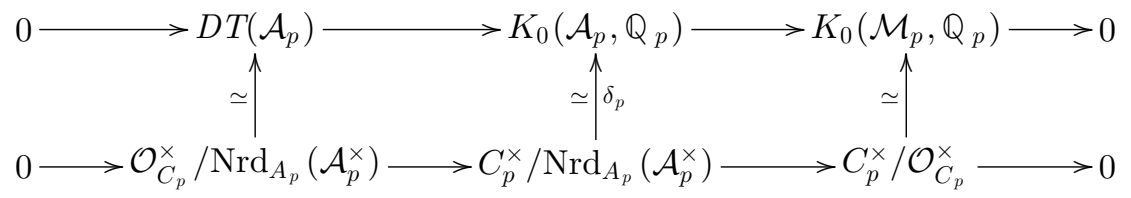

FIGURE 2. Algebraic K-groups and reduced norms.

The primitive idempotents of $C$ will be denoted by $e_{1}, \ldots, e_{r}$. For $i=1, \ldots, r$ we set $A_{i}:=A e_{i}$. Then

$$
A=A_{1} \oplus \cdots \oplus A_{r}
$$

is a decomposition into indecomposable ideals $A_{i}$ of $A$. Each $A_{i}$ is a $\mathbb{Q}$-algebra with identity element $e_{i}$. The centers $K_{i}:=\zeta\left(A_{i}\right)$ are finite field extensions of $\mathbb{Q}$ via $\mathbb{Q} \rightarrow K_{i}, \alpha \mapsto \alpha e_{i}$, and we have $\mathbb{Q}$-algebra isomorphisms $A_{i} \simeq \operatorname{Mat}_{n_{i}}\left(D_{i}\right)$ for each $i=1, \ldots, r$, where $D_{i}$ is a skew field with $\zeta\left(D_{i}\right) \simeq K_{i}$. The Wedderburn decomposition induces decompositions

$$
C=K_{1} \oplus \cdots \oplus K_{r}, \quad \mathcal{O}_{C}=\mathcal{O}_{K_{1}} \oplus \cdots \oplus \mathcal{O}_{K_{r}}
$$

Let $\mathfrak{f}$ be a full, two-sided ideal of $\mathcal{M}$ contained in $\mathcal{A}$ and put $\mathfrak{g}:=\mathcal{O}_{C} \cap \mathfrak{f}$. Since $\mathcal{M}$ contains the idempotents $e_{i}$, the ideal $\mathfrak{f}$ of $\mathcal{M}$ decomposes into $\mathfrak{f}=\mathfrak{f}_{1} \oplus \cdots \oplus \mathfrak{f}_{r}$, and similarly, $\mathfrak{g}=\mathfrak{g}_{1} \oplus \cdots \oplus \mathfrak{g}_{r}$. By [Bley and Wilson 09, Theorem 2.6], the reduced norm $\mathrm{Nrd}_{A}$ on $A$ induces a homomorphism

$$
\mu: K_{1}(\mathcal{A} / \mathfrak{f}) \longrightarrow\left(\mathcal{O}_{C} / \mathfrak{g}\right)^{\times}
$$

and a canonical isomorphism $D T(\mathcal{A}) \simeq \operatorname{cok}(\mu)$, where $D T(\mathcal{A})$ denotes the torsion subgroup of $K_{0}(\mathcal{A}, \mathbb{Q})$. This isomorphism encodes certain congruences implied by the triviality of an element in $D T(\mathcal{A})$.

For a rational prime $p$, one has canonical isomorphisms

$$
A_{p} \simeq \bigoplus A_{i, \mathfrak{P}}
$$

where for given $i \in\{1, \ldots, r\}, \mathfrak{P}$ runs through all maximal ideals of $\mathcal{O}_{K_{i}}$ dividing $p$, and $A_{i, \mathfrak{P}}$ is defined as $A_{i} \otimes_{K_{i}} K_{i, \mathfrak{P}}$, where $K_{i, \mathfrak{P}}$ denotes completion of $K_{i}$ with respect to $\mathfrak{P}$. Similarly, we have a canonical isomorphism

$$
C_{p} \simeq \bigoplus K_{i, \mathfrak{P}}
$$

We write $I_{p}(C)$ for the group of fractional ideals of $C$ with support above $p$. Then Figure 2 together with (2-3) induces a canonical identification of $K_{0}\left(\mathcal{M}_{p}, \mathbb{Q}_{p}\right)$ with $I_{p}(C)$.
We put $\mathfrak{a}_{p}$ for the $p$-primary part of a fractional ideal a. Then one has a homomorphism

$$
\mu_{p}: K_{1}\left(\mathcal{A}_{p} / \mathfrak{f}_{p}\right) \longrightarrow\left(\mathcal{O}_{C_{p}} / \mathfrak{g}_{p}\right)^{\times} \simeq \bigoplus_{i=1}^{r}\left(\mathcal{O}_{K_{i}} / \mathfrak{g}_{i, p}\right)^{\times}
$$

and a canonical isomorphism

$$
D T\left(\mathcal{A}_{p}\right) \simeq \operatorname{cok}\left(\mu_{p}\right)
$$

Combined with the isomorphism $\delta_{p}$ from Figure 2, this is the origin of explicit congruences. See the next section for even more explicit versions of these congruences in the case of cyclic groups $Z_{p}$, dihedral groups $D_{2 p}$, and the alternating group $A_{4}$.

We obtain the following canonical short exact sequence:

$$
0 \longrightarrow \operatorname{cok}\left(\mu_{p}\right) \longrightarrow K_{0}\left(\mathcal{A}_{p}, \mathbb{Q}_{p}\right) \longrightarrow I_{p}(C) \longrightarrow 0 .
$$

After choosing an explicit splitting, we have by [Bley and Wilson 09, Proposition 2.7] a noncanonical isomorphism

$$
K_{0}\left(\mathcal{A}_{p}, \mathbb{Q}_{p}\right) \simeq \operatorname{cok}\left(\mu_{p}\right) \oplus I_{p}(C) .
$$

In [Bley and Wilson 09, Sections 3 and 4] it is shown how the right-hand side can be computed explicitly and how to solve the logarithm problem. We briefly recall the logarithm algorithm; for the details we refer the reader to [Bley and Wilson 09, Section 4.1].

Let $\eta=[P, \varphi, Q] \in K_{0}\left(\mathcal{A}_{p}, \mathbb{Q}_{p}\right)$. By Remark 2.7(a), we may without loss of generality assume that $P$ and $Q$ are $\mathcal{A}_{p}$-free. Then one computes $\mathcal{A}_{p}$-bases $\nu_{1}, \ldots, \nu_{d}$ and $\omega_{1}, \ldots, \omega_{d}$ of $P$ and $Q$ and a matrix $S \in \mathrm{Gl}_{d}\left(A_{p}\right)$ such that $\left(\varphi\left(\nu_{1}\right), \ldots, \varphi\left(\nu_{d}\right)\right)=\left(\omega_{1}, \ldots, \omega_{d}\right) S$. In all of our applications we will be able to choose $S \in \mathrm{Gl}_{d}(A)$. If $\tilde{\eta}:=\operatorname{Nrd}_{A}(S)$, then $\tilde{\eta}$ represents $\eta$ via the middle vertical isomorphism in Figure 2, and we will use [Bley and Wilson 09, Proposition 2.7] to read $\tilde{\eta}$ in $\operatorname{cok}\left(\mu_{p}\right) \oplus I_{p}(C)$. In particular, we have a test whether $[P, \varphi, Q]=0$ in $K_{0}\left(\mathcal{A}_{p}, \mathbb{Q}_{p}\right)$. If $\tilde{\eta}=\left(\eta_{1}, \ldots, \eta_{r}\right)$ with $\eta_{i} \in$ $K_{i}$, then

$$
[P, \varphi, Q]=0 \Longleftrightarrow v_{\mathfrak{P}}\left(\eta_{i}\right)=0, \forall i \in\{1, \ldots, r\} \text { with } \mathfrak{P} \mid p
$$
and $\left(\bar{\eta}_{1}, \ldots \bar{\eta}_{r}\right) \in \operatorname{im}\left(\mu_{p}\right)$, 
where $\bar{\eta}_{i}$ is the image of $\eta_{i}$ under the projection $\mathcal{O}_{K_{i}, p}^{\times} \longrightarrow$ $\left(\mathcal{O}_{K_{i}} / \mathfrak{g}_{i, p}\right)^{\times}$.

Example 2.8. Let $p$ be a prime and let $T$ be a finite perfect $\mathbb{Z}_{p}[G]$-module. Then $T$ is also perfect as a $\mathbb{Z}[G]$-module and we may choose a $\mathbb{Z}[G]$-projective resolution of the form

$$
0 \longrightarrow Q \stackrel{\alpha}{\longrightarrow} P \longrightarrow T \longrightarrow 0 \text {. }
$$

By a fundamental result of Swan (see [Curtis and Reiner 87, Theorem (32.11)]), the projectives $P$ and $Q$ are locally free, and we can therefore apply the algorithm of [Bley and Wilson 09, Section 4.2$]$ to compute $\mathbb{Z}_{p}[G]$-bases $\nu_{1}, \ldots, \nu_{d}$ of $Q \otimes_{\mathbb{Z}} \mathbb{Z}_{p}$ and $\omega_{1}, \ldots, \omega_{d}$ of $P \otimes_{\mathbb{Z}} \mathbb{Z}_{p}$. The algorithm of [Bley and Wilson 09] actually produces elements $\nu_{i} \in Q$ and $\omega_{j} \in P$. Hence we obtain a matrix $S \in \mathrm{Gl}_{d}(A)$ that represents $\alpha$. Then $\tilde{\eta}:=\operatorname{Nrd}_{A}(S) \in C^{\times} \subseteq C_{p}^{\times}$represents $\chi_{\mathbb{Z}_{p}[G], \mathbb{Q}_{p}}(T) \in K_{0}\left(\mathbb{Z}_{p}[G], \mathbb{Q}_{p}\right)$.

Note that in the case $\# G=1$, this just generalizes the notion of the order of the $p$-primary part of $T$.

We conclude this section by explicitly describing the element

$$
\Omega_{p}=\left[X_{p}^{\mathrm{even}}, \theta_{p}^{\prime}, X_{p}^{\mathrm{odd}}\right]-\delta_{p}\left(j_{*}(\mathcal{L} *)\right)
$$

from Proposition 2.6. We assume that $v_{1}, \ldots, v_{d}$ and $w_{1}, \ldots, w_{d}$ constitute $\mathbb{Z}_{p}[G]$-bases respectively of $X_{p}^{\text {even }}$ and $X_{p}^{\text {odd }}$. Then $\Omega_{p}$ is represented by

$$
\operatorname{Nrd}_{\mathbb{C}_{p}[G]}(B) / j_{*}\left(\mathcal{L}^{*}\right) .
$$

By Lemma 2.5, the element $\Omega_{p}$ is rational if and only if

$$
\operatorname{Nrd}_{\mathbb{C}_{p}[G]}(B) / j_{*}\left(\mathcal{L}^{*}\right) \in \zeta\left(\mathbb{Q}_{p}[G]\right)^{\times} .
$$

If this is the case, we can interpret $\operatorname{Nrd}_{\mathbb{C}_{p}[G]}(B) / j_{*}\left(\mathcal{L}^{*}\right)=$ $\left(\eta_{1}, \ldots, \eta_{r}\right)$ in $\operatorname{cok}\left(\mu_{p}\right) \oplus I_{p}(C)$ and thus determine the image of $\Omega_{p}$ in $\operatorname{cok}\left(\mu_{p}\right) \oplus I_{p}(C)$.

In our applications the modules $X_{p}^{\text {even }} \otimes_{\mathbb{Z}_{p}} \mathbb{Q}_{p}$ and $X_{p}^{\text {odd }} \otimes_{\mathbb{Z}_{p}} \mathbb{Q}_{p}$ are usually of the form $X^{\text {even }} \otimes_{\mathbb{Z}} \mathbb{Q}_{p}$ and $X^{\text {odd }} \otimes_{\mathbb{Z}} \mathbb{Q}_{p}$ with finitely generated $\mathbb{Z}[G]$-modules $X^{\text {even }}$ and $X^{\text {odd }}$. In this case, the rationality question can be treated by studying the quotient $\operatorname{Nrd}_{\mathbb{R}[G]}(B) / \mathcal{L}^{*}$, where $B$ is a matrix computed with respect to any choice of $\mathbb{Q}[G]$-bases.

It remains to explain how we actually perform the test $\operatorname{Nrd}_{\mathbb{R}[G]}(B) / \mathcal{L}^{*} \in \zeta(\mathbb{Q}[G])^{\times}$. Let $\operatorname{Irr}(G)$ denote the set of absolutely irreducible characters. By Wedderburn's theorem, $\mathbb{C}[G] \simeq \prod_{\chi \in \operatorname{Irr}(G)} M_{n_{\chi}}(\mathbb{C})$, which induces a canonical isomorphism $\zeta(\mathbb{C}[G]) \simeq \prod_{\chi \in \operatorname{Irr}(G)} \mathbb{C}$. Explicitly,

$$
\prod_{\chi \in \operatorname{Irr}(G)} \mathbb{C} \longrightarrow \zeta(\mathbb{C}[G]), \quad\left(\alpha_{\chi}\right)_{\chi \in \operatorname{Irr}(G)} \mapsto \sum_{\chi \in \operatorname{Irr}(G)} \alpha_{\chi} e_{\chi},
$$

with the central primitive idempotents

$$
e_{\chi}=\frac{\chi(1)}{|G|} \sum_{g \in G} \chi(g) g^{-1} \text {. }
$$

Lemma 2.9. Let $\mathbb{Q} \subseteq F \subseteq \mathbb{C}$ and let

$$
\alpha=\left(\alpha_{\chi}\right)_{\chi \in \operatorname{Irr}(G)} \in \prod_{\chi \in \operatorname{Irr}(G)} \mathbb{C} \simeq \zeta(\mathbb{C}[G]) .
$$

Then one has

$$
\alpha \in \zeta(F[G]) \Longleftrightarrow \alpha_{\sigma \circ \chi}=\sigma\left(\alpha_{\chi}\right)
$$

for all $\chi \in \operatorname{Irr}(G)$ and all $\sigma \in \operatorname{Aut}(\mathbb{C} / F)$.

Proof. We have to show that $\sum_{\chi \in \operatorname{Irr}(G)} \alpha_{\chi} e_{\chi} \in F[G]$ if and only if $\alpha_{\sigma \circ \chi}=\sigma\left(\alpha_{\chi}\right)$ for all $\chi \in \operatorname{Irr}(G)$ and all $\sigma \in$ $\operatorname{Aut}(\mathbb{C} / F)$. This amounts to showing that

$$
\sum_{\chi \in \operatorname{Irr}(G)} \chi(1) \alpha_{\chi} \chi(g) \in F, \forall g \Longleftrightarrow \alpha_{\sigma \circ \chi}=\sigma\left(\alpha_{\chi}\right), \forall \chi, \sigma .
$$

If $\sum_{\chi} \chi(1) \alpha_{\chi} \chi(g) \in F, \forall g \in G$, then we easily show that

$$
\sum_{\chi \in \operatorname{Irr}(G)} \chi(1)\left(\alpha_{\sigma \circ \chi}-\sigma\left(\alpha_{\chi}\right)\right)(\sigma \circ \chi)(g)=0
$$

for all $g \in G$. The assertion now follows from the linear independence of absolutely irreducible characters.

Conversely, we deduce that

$$
\sigma\left(\sum_{\chi \in \operatorname{Irr}(G)} \chi(1) \alpha_{\chi} \chi(g)\right)=\sum_{\chi \in \operatorname{Irr}(G)} \chi(1) \alpha_{\chi} \chi(g)
$$

for all $\sigma \in \operatorname{Aut}(\mathbb{C} / F)$. Since for any $\beta \in \mathbb{C}$ one has

$$
\beta \in F \Longleftrightarrow \sigma(\beta)=\beta, \quad \forall \sigma \in \operatorname{Aut}(\mathbb{C} / F),
$$

the result follows.

For $\chi \in \operatorname{Irr}(G)$ we write $\mathbb{Q}(\chi)$ for the extension generated over $\mathbb{Q}$ by the values of $\chi$. We recall that $\mathbb{Q}(\chi) / \mathbb{Q}$ is an abelian extension. By Lemma 2.9, one has

$$
\alpha \in \zeta(\mathbb{Q}[G]) \Longleftrightarrow \alpha_{\chi} \in \mathbb{Q}(\chi) \text { and } \alpha_{\sigma \circ \chi}=\sigma\left(\alpha_{\chi}\right)
$$

for all $\chi \in \operatorname{Irr}(G)$ and all $\sigma \in \operatorname{Gal}(\mathbb{Q}(\chi) / \mathbb{Q})$. This can be efficiently checked if we have good approximations of the complex numbers $\alpha_{\chi}$ and bounds for the denominators.

We fix a set $\operatorname{Irr}_{\mathbb{Q}}(G) \subseteq \operatorname{Irr}(G)$ of representatives of $\operatorname{Irr}(G)$ modulo the action of $\operatorname{Aut}(\mathbb{C} / \mathbb{Q})$. Thus we identify $C$ with $\prod_{\chi \in \operatorname{Irr}_{\mathbb{Q}}(G)} \mathbb{Q}(\chi)$. Once we know or trust in the validity of the rationality conjecture, we will work in $\prod_{\chi \in \operatorname{Irr}_{\mathbb{Q}}(G)} \mathbb{Q}(\chi)$. Note that the fields $K_{i}, i=1, \ldots, r$, can be identified with the character fields $\mathbb{Q}(\chi), \chi \in \operatorname{Irr}_{\mathbb{Q}}(G)$. 


\subsection{Explicit Congruences}

Assume that

$$
\alpha=\left(\alpha_{\chi}\right)_{\chi} \in \prod_{\chi \in \operatorname{Irr}_{\mathbb{Q}}(G)} \mathbb{Q}(\chi) \simeq C^{\times}
$$

We let $p$ denote an odd prime. In this section we will consider the cyclic groups $Z_{p}$, the dihedral groups $D_{2 p}$, and the alternating group $A_{4}$ and rephrase the condition $\delta_{p}(\alpha)=0$ in $K_{0}\left(\mathbb{Z}_{p}[G], \mathbb{Q}_{p}\right)$ in terms of explicit congruences. On the one hand, this serves as an explicit illustration of the methods introduced in [Bley and Wilson 09], while on the other hand it leads to very remarkable congruences that are conjecturally satisfied by the twisted BSD quotients (see Remark 4.6).

Let $p$ be a rational prime and $G$ an arbitrary finite group. We adopt the notation from the previous subsection. Let $\mathfrak{f}:=\{\lambda \in \mathbb{Q}[G] \mid \lambda \mathcal{M} \subseteq \mathbb{Z}[G]\}$ be the conductor of $\mathbb{Z}[G]$ in $\mathcal{M}$. Let $\mathfrak{g}:=\mathfrak{f} \cap \mathcal{O}_{C}$ be the central conductor. Note that we make use of an explicit formula for $\mathfrak{g}$ in [Curtis and Reiner 87, Theorem 27.13].

We recall that

$$
\delta_{p}(\alpha) \in D T\left(\mathbb{Z}_{p}[G]\right) \Longleftrightarrow \alpha_{\chi} \in \mathcal{O}_{\mathbb{Q}}^{\times}(\chi), p
$$

for all $\chi \in \operatorname{Irr}_{\mathbb{Q}}(G)$. The explicit congruences are encoded in the canonical isomorphism (2-4). We will make this explicit for the groups $Z_{p}, D_{2 p}$, and $A_{4}$.

Let $G=\left\langle g_{0}\right\rangle$ be cyclic of order $p$. Let $\zeta_{p}$ be a fixed primitive $p$ th root of unity and define irreducible characters $\chi_{0}$ and $\chi_{1}$ by $\chi_{0}\left(g_{0}\right)=1$ and $\chi_{1}\left(g_{0}\right)=\zeta_{p}$. Then

$$
\mathbb{Q}[G] \simeq \mathbb{Q} \oplus \mathbb{Q}\left(\zeta_{p}\right), \quad \lambda \mapsto\left(\chi_{0}(\lambda), \chi_{1}(\lambda)\right)
$$

Let $\alpha=\left(\alpha_{0}, \alpha_{1}\right) \in \mathbb{Q}^{\times} \oplus \mathbb{Q}\left(\zeta_{p}\right)^{\times}$be a $p$-adic unit (i.e., $\left.\alpha \in \mathcal{O}_{C_{p}}^{\times}\right)$. Then

$$
\begin{aligned}
\delta_{p}\left(\left(\alpha_{0}, \alpha_{1}\right)\right) & =0 \text { in } D T\left(\mathbb{Z}_{p}\left[Z_{p}\right]\right) \\
\Longleftrightarrow \alpha_{0} & \equiv \alpha_{1}\left(\bmod \left(1-\zeta_{p}\right)\right) .
\end{aligned}
$$

For a proof and the generalization for cyclic groups of prime-power order we refer the reader to [Bley 10, Section 5].

Let now

$$
G=\left\langle\sigma, \tau \mid \sigma^{p}=\tau^{2}=1, \tau \sigma=\sigma^{-1} \tau\right\rangle
$$

be the dihedral group $D_{2 p}$ for an odd prime $p$. Then $\mathbb{Q}[G] \simeq \mathbb{Q} \oplus \mathbb{Q} \oplus M_{2}\left(\mathbb{Q}\left(\zeta_{p}\right)^{+}\right)$, and we fix such an iso- morphism by

$$
\begin{gathered}
\sigma \mapsto\left(1,1,\left(\begin{array}{cc}
0 & -1 \\
1 & \zeta_{p}+\zeta_{p}^{-1}
\end{array}\right)\right), \\
\tau \mapsto\left(1,-1,\left(\begin{array}{cc}
1 & \zeta_{p}+\zeta_{p}^{-1} \\
0 & -1
\end{array}\right)\right) .
\end{gathered}
$$

From [Bley and Wilson 09, Theorem 1.1], we know that $D T\left(\mathbb{Z}_{p}[G]\right) \simeq Z_{p-1}$ (where for $n \in \mathbb{N}$ we write $Z_{n}$ for the cyclic group of order $n$ ). Let $H=\langle\sigma\rangle$. By [Breuning 04, Proposition 3.2], we know that the restriction map

$$
\text { res: } D T\left(\mathbb{Z}_{p}[G]\right) \longrightarrow D T\left(\mathbb{Z}_{p}[H]\right)
$$

is injective. Let $\alpha=\left(\alpha_{0}, \alpha_{1}, \alpha_{2}\right) \in \mathbb{Q}^{\times} \oplus \mathbb{Q}^{\times} \oplus \mathbb{Q}\left(\zeta_{p}\right)^{+\times}$ be a $p$-adic unit. By [Breuning 04, Lemma 3.9] or [Bley and Burns 03, p. 575] one has $\operatorname{res}(\alpha)=\left(\alpha_{0} \alpha_{1}, \alpha_{2}\right)$, so that we conclude from the result for cyclic groups $Z_{p}$ that

$$
\begin{aligned}
& \delta_{p}\left(\left(\alpha_{0}, \alpha_{1}, \alpha_{2}\right)\right)=0 \text { in } D T\left(\mathbb{Z}_{p}\left[D_{2 p}\right]\right) \\
& \Longleftrightarrow \alpha_{0} \alpha_{1} \equiv \alpha_{2}(\bmod \mathfrak{p}),
\end{aligned}
$$

where $\mathfrak{p}$ denotes the unique prime ideal of $\mathbb{Q}\left(\zeta_{p}\right)^{+}$over the rational prime $p$. It is also well known (see, e.g., [Breuning 04, Proposition 3.2]) that $D T\left(\mathbb{Z}_{2}\left[D_{2 p}\right]\right)$ is trivial.

Let now $G$ be the alternating group $A_{4}$. If $\sigma=$ $(1,2)(3,4)$ and $\nu=(1,2,3)$, then $G=\langle\sigma, \nu\rangle$. We have $\mathbb{Q}[G] \simeq \mathbb{Q} \oplus \mathbb{Q}\left(\zeta_{3}\right) \oplus M_{3}(\mathbb{Q})$, and we fix such an isomorphism by

$$
\begin{gathered}
\sigma \mapsto\left(1,1,\left(\begin{array}{ccc}
1 & 0 & 0 \\
0 & -1 & 0 \\
0 & 0 & -1
\end{array}\right)\right), \\
\nu \mapsto\left(1, \zeta_{3},\left(\begin{array}{lll}
0 & 1 & 0 \\
0 & 0 & 1 \\
1 & 0 & 0
\end{array}\right)\right) .
\end{gathered}
$$

From [Bley and Wilson 09, Theorem 1.2], we know that $D T\left(\mathbb{Z}_{2}[G]\right) \simeq Z_{2}$ and $D T\left(\mathbb{Z}_{3}[G]\right) \simeq Z_{2}$. We have $\mathcal{O}_{C}=$ $\left(\mathbb{Z}, \mathbb{Z}\left[\zeta_{3}\right], \mathbb{Z}\right)$ and $\mathfrak{g}=\left(12 \mathbb{Z}, 4\left(1-\zeta_{3}\right), 4 \mathbb{Z}\right)$. We first consider $p=3$. Clearly

$$
\left(\mathcal{O}_{C} / \mathfrak{g}_{3}\right)^{\times} \simeq(\mathbb{Z} / 3 \mathbb{Z})^{\times} \oplus\left(\mathbb{Z}\left[\zeta_{3}\right] /\left(1-\zeta_{3}\right)\right)^{\times} \simeq Z_{2} \times Z_{2} .
$$

Since

$$
\operatorname{Nrd}_{\mathbb{Q}[G]}(1+\nu)=\left(2,1+\zeta_{3}, 2\right) \equiv(-1,-1)\left(\bmod \mathfrak{g}_{3}\right),
$$

it follows that $\operatorname{im}\left(\mu_{3}\right)=\{(1,1),(-1,-1)\}$, and we obtain

$$
\begin{gathered}
\delta_{3}\left(\left(\alpha_{0}, \alpha_{1}, \alpha_{2}\right)\right)=0 \text { in } D T\left(\mathbb{Z}_{3}\left[A_{4}\right]\right) \\
\Longleftrightarrow \alpha_{1} \equiv \alpha_{0}\left(\bmod \left(1-\zeta_{3}\right)\right) .
\end{gathered}
$$


For $p=2$ one has

$$
\begin{aligned}
\left(\mathcal{O}_{C} / \mathfrak{g}_{2}\right)^{\times} & \simeq(\mathbb{Z} / 4 \mathbb{Z})^{\times} \oplus\left(\mathbb{Z}\left[\zeta_{3}\right] /(4)\right)^{\times} \oplus(\mathbb{Z} / 4 \mathbb{Z})^{\times} \\
& \simeq Z_{2} \times\left(Z_{2} \times Z_{6}\right) \times Z_{2} .
\end{aligned}
$$

By explicit computation we show that

$$
\begin{aligned}
\operatorname{Nrd}_{\mathbb{Q}[G]}(2+\nu) & \equiv\left(-1,2+\zeta_{3}, 1\right)\left(\bmod \mathfrak{g}_{2}\right), \\
\operatorname{Nrd}_{\mathbb{Q}[G]}(-1+2 \nu) & \equiv\left(1,-1+2 \zeta_{3},-1\right)\left(\bmod \mathfrak{g}_{2}\right), \\
\operatorname{Nrd}_{\mathbb{Q}[G]}(-\sigma) & \equiv(-1,-1,-1)\left(\bmod \mathfrak{g}_{2}\right) .
\end{aligned}
$$

Again by explicit computation one verifies that the classes of $\operatorname{Nrd}_{\mathbb{Q}[G]}(2+\nu), \operatorname{Nrd}_{\mathbb{Q}[G]}(-1+2 \nu)$, and $\operatorname{Nrd}_{\mathbb{Q}[G]}(-\sigma)$ generate the kernel of the surjective homomorphism

$$
\begin{aligned}
\left(\mathcal{O}_{C} / \mathfrak{g}_{2}\right)^{\times} & \longrightarrow(\mathbb{Z} / 4 \mathbb{Z})^{\times}, \\
\left(\alpha_{0}, \alpha_{1}, \alpha_{2}\right) & \mapsto \alpha_{0} N_{\mathbb{Q}\left(\zeta_{3}\right) / \mathbb{Q}}\left(\alpha_{1}\right) \alpha_{2} .
\end{aligned}
$$

Together with $D T\left(\mathbb{Z}_{2}[G]\right) \simeq Z_{2}$, it follows that $\operatorname{im}\left(\mu_{2}\right)$ equals this kernel, so that

$$
\begin{aligned}
& \delta_{2}\left(\left(\alpha_{0}, \alpha_{1}, \alpha_{2}\right)\right)=0 \text { in } D T\left(\mathbb{Z}_{2}\left[A_{4}\right]\right) \\
& \quad \Longleftrightarrow \alpha_{0} N_{\mathbb{Q}\left(\zeta_{3}\right) / \mathbb{Q}}\left(\alpha_{1}\right) \equiv \alpha_{2}(\bmod 4) .
\end{aligned}
$$

\section{THE EQUIVARIANT TAMAGAWA NUMBER CONJECTURE FOR THE BASE CHANGE OF AN ELLIPTIC CURVE}

Let $K / \mathbb{Q}$ be a finite Galois extension with group $G$. Let $E$ be an elliptic curve defined over $\mathbb{Q}$. We denote the base change $\operatorname{Spec}(K) \times_{\operatorname{Spec}(\mathbb{Q})} E$ by $E_{K}$ and consider $M_{K}=h^{1}\left(E_{K}\right)(1)$ as a motive over $\mathbb{Q}$. The Galois group $G$ naturally acts on $M_{K}$ via the first factor, and thus we have a natural action of $A=\mathbb{Q}[G]$ on the realizations and motivic cohomology groups attached to $M_{K}$. For an explicit description of the realizations we refer the reader to [Burns 09, Section 4.1].

The purpose of this section is to provide an explicit description of the equivariant Tamagawa number conjecture (ETNC) for the pair $\left(M_{K}, \mathbb{Z}[G]\right)$. Our main reference is [Burns and Flach 01], from which we adopt most of our notation. Further references are the survey articles [Flach 04, Flach 09, Venjakob 07].

We first note that by Poincaré duality, the dual motive $M_{K}^{*}(1)$ identifies with $M_{K}$. The motivic cohomology spaces $H_{f}^{0}\left(M_{K}\right)$ and $H_{f}^{1}\left(M_{K}\right)$ are given by

$$
H_{f}^{0}\left(M_{K}\right)=0, \quad H_{f}^{1}\left(M_{K}\right)=E(K) \otimes_{\mathbb{Z}} \mathbb{Q},
$$

where as usual, $E(K)$ denotes the Mordell-Weil group of $E / K$.

For a number field $F$ we write $G_{F}$ for the absolute Galois group. Let $v$ be a place of $K$. We write $K_{v}$ for the completion of $K$ at $v$, and fix an algebraic closure $\bar{K}_{v}$ of $K_{v}$ and an embedding $\bar{K}$ into $\bar{K}_{v}$. We denote by $G_{v} \subseteq G_{K}$ the corresponding decomposition group, and if $v$ is nonarchimedean, by $I_{v} \subseteq G_{v}$ the inertia group. We write $\operatorname{Fr}_{v} \in G_{v} / I_{v}$ for the Frobenius substitution.

For any number field $F$ we let $\Sigma(F)$ denote the set of embeddings of $F$ into $\mathbb{C}$. We define $H_{K}:=\bigoplus_{\Sigma(K)} \mathbb{Q}$. The groups $G$ and $\operatorname{Gal}(\mathbb{C} / \mathbb{R})$ act on $\Sigma(K)$ and endow $H_{K}$ with the structure of a $(\operatorname{Gal}(\mathbb{C} / \mathbb{R}) \times G)$-module. Let $\left\{w_{j}: j \in \Sigma(K)\right\}$ denote the canonical $\mathbb{Q}$-basis of $H_{K}$. We write $c \in \operatorname{Gal}(\mathbb{C} / \mathbb{R})$ for complex conjugation. Then $c w_{j}=w_{c \circ j}$ and $\sigma w_{j}=w_{j \circ \sigma^{-1}}$ for $\sigma \in G$.

For any commutative ring $R$ and any $R[\operatorname{Gal}(\mathbb{C} / \mathbb{R})]$ module $X$ we write $X^{+}$and $X^{-}$for the submodules on which complex conjugation acts by +1 and -1 , respectively.

We write $\rho_{K}: \mathbb{C} \otimes_{\mathbb{Q}} K \longrightarrow \mathbb{C} \otimes_{\mathbb{Q}} H_{K}$ for the canonical $\mathbb{C}[\operatorname{Gal}(\mathbb{C} / \mathbb{R}) \times G]$-equivariant isomorphism that is induced by $z \otimes \alpha \mapsto(z j(\alpha))_{j \in \Sigma(K)}$. Let $\tilde{\rho}_{K}: \mathbb{R} \otimes_{\mathbb{Q}}$ $K \longrightarrow \mathbb{R} \otimes_{\mathbb{Q}} H_{K}$ be the $\mathbb{R}[G]$-equivariant isomorphism defined in [Bley and Burns 03, p. 554], where it is denoted by $\pi_{K}$.

We write $\infty$ for the archimedean place of $\mathbb{Q}$ and let $S_{\infty}(K)$ denote the set of archimedean places of $K$. For each $v \in S_{\infty}(K)$ we choose $\sigma_{v} \in \Sigma(K)$ corresponding to $v$. Since $E$ is defined over $\mathbb{Q}$, one has $\sigma E_{K}=E_{K}$ for all $\sigma \in \Sigma(K)$. As usual, we write $M_{B}$ for the Betti realization, that is,

$$
\begin{aligned}
M_{B} & =\bigoplus_{\sigma \in \Sigma(K)} H^{1}\left(\sigma E_{K}(\mathbb{C}), 2 \pi i \mathbb{Q}\right) \\
& =H_{K} \otimes_{\mathbb{Q}} H^{1}(E(\mathbb{C}), 2 \pi i \mathbb{Q}) .
\end{aligned}
$$

By identifying $H^{1}(E(\mathbb{C}), 2 \pi i \mathbb{Q})$ with the dual homology $\mathcal{H}:=\operatorname{Hom}_{\mathbb{Q}}\left(H_{1}(E(\mathbb{C}), \mathbb{Q}), 2 \pi i \mathbb{Q}\right)$, we obtain

$$
M_{B}^{+} \simeq \bigoplus_{v \in S_{\infty}(K)} H^{1}\left(\sigma_{v} E_{K}(\mathbb{C}), 2 \pi i \mathbb{Q}\right)^{G_{v}} \simeq\left[H_{K} \otimes_{\mathbb{Q}} \mathcal{H}\right]^{+} .
$$

We write $M_{\mathrm{dR}}$ for the de Rham realization,

$$
M_{\mathrm{dR}}=H_{\mathrm{dR}}^{1}(E / K),
$$

with the natural decreasing filtration $\left\{F^{i} H_{\mathrm{dR}}^{1}(E / K)\right\}_{i \in \mathbb{Z}}$ shifted by 1 . Thus

$$
\begin{aligned}
M_{\mathrm{dR}} / M_{\mathrm{dR}}^{0} & =H_{\mathrm{dR}}^{1}(E / K) / F^{1} H_{\mathrm{dR}}^{1}(E / K) \\
& \simeq H^{1}\left(E_{K}, \mathcal{O}_{E_{K}}\right) .
\end{aligned}
$$

The $G$-module $H^{1}\left(E_{K}, \mathcal{O}_{E_{K}}\right)$ is isomorphic to $K \otimes_{\mathbb{Q}}$ $H^{1}\left(E, \mathcal{O}_{E}\right)$. Now $H^{1}\left(E, \mathcal{O}_{E}\right)$ is canonically isomorphic to $\Omega_{E}^{1}(E)^{*}:=\operatorname{Hom}\left(\Omega_{E}^{1}(E), \mathbb{Q}\right)$, so that we finally make the identification

$$
M_{\mathrm{dR}} / M_{\mathrm{dR}}^{0} \simeq K \otimes_{\mathbb{Q}} \Omega_{E}^{1}(E)^{*} .
$$


We let $\omega_{0}$ denote a Néron differential and let $\gamma_{+}$and $\gamma_{-}$be $\mathbb{Z}$-generators of $H_{1}(E(\mathbb{C}), \mathbb{Z})^{+}$and $H_{1}(E(\mathbb{C}), \mathbb{Z})^{-}$, respectively. We define

$$
\Omega_{+}:=\int_{\gamma_{+}} \omega_{0}, \quad \Omega_{-}:=\int_{\gamma_{-}} \omega_{0} .
$$

We write $\omega_{0}^{*} \in \Omega_{E}^{1}(E)^{*}$ for the map that sends $\omega_{0}$ to 1 . Similarly, we define $\mathbb{Q}$-linear maps $\gamma_{+}^{*}, \gamma_{-}^{*} \in \mathcal{H}$ by

$$
\begin{aligned}
& \gamma_{+}^{*}\left(\gamma_{+}\right)=2 \pi i, \quad \gamma_{+}^{*}\left(\gamma_{-}\right)=0, \\
& \gamma_{-}^{*}\left(\gamma_{+}\right)=0, \quad \gamma_{-}^{*}\left(\gamma_{-}\right)=2 \pi i .
\end{aligned}
$$

For $\gamma=a \gamma_{+}+b \gamma_{-} \in H_{1}(E(\mathbb{C}), \mathbb{Q}), \quad a, b \in \mathbb{Q}$, we set $\gamma^{*}:=a \gamma_{+}^{*}+b \gamma_{-}^{*}$. Finally, we define

$$
\begin{gathered}
\pi: \mathbb{C} \otimes_{\mathbb{Q}} \mathcal{H} \longrightarrow \mathbb{C} \otimes_{\mathbb{Q}} \Omega_{E}^{1}(E)^{*}, \\
z \otimes \gamma^{*} \mapsto\left(\omega \mapsto z \int_{\gamma} \omega\right) .
\end{gathered}
$$

We write $\pi_{K}: \mathbb{R} \otimes_{\mathbb{Q}} M_{B}^{+} \longrightarrow \mathbb{R} \otimes_{\mathbb{Q}} M_{\mathrm{dR}} / M_{\mathrm{dR}}^{0}$ for the period isomorphism. Then $\pi_{K}$ is explicitly given by the following composite of $\mathbb{R}[G]$-equivariant maps:

$$
\begin{aligned}
\mathbb{R} \otimes_{\mathbb{Q}} & {\left[H_{K} \otimes_{\mathbb{Q}} \mathcal{H}\right]^{+} } \\
= & \mathbb{R} \otimes_{\mathbb{Q}} H_{K}^{+} \otimes_{\mathbb{Q}} \mathcal{H}^{+} \oplus \mathbb{R} \otimes_{\mathbb{Q}} H_{K}^{-} \otimes_{\mathbb{Q}} \mathcal{H}^{-} \\
= & \left(\mathbb{R} \otimes_{\mathbb{Q}} H_{K}^{+}\right) \otimes_{\mathbb{R}}\left(\mathbb{R} \otimes_{\mathbb{Q}} \mathcal{H}^{+}\right) \oplus\left(\mathbb{R} \otimes_{\mathbb{Q}} H_{K}^{-}\right) \\
& \otimes_{\mathbb{R}}\left(\mathbb{R} \otimes_{\mathbb{Q}} \mathcal{H}^{-}\right) \\
& \left(\stackrel{\mathrm{id} \otimes \pi, \mathrm{id} \otimes \pi)}{\longrightarrow}\left(\mathbb{R} \otimes_{\mathbb{Q}} H_{K}^{+}\right) \otimes_{\mathbb{R}}\left(\mathbb{R} \otimes_{\mathbb{Q}} \Omega_{E}^{1}(E)^{*}\right)\right. \\
& \oplus\left(\mathbb{R} \otimes_{\mathbb{Q}} H_{K}^{-}\right) \otimes_{\mathbb{R}}\left(i \mathbb{R} \otimes_{\mathbb{Q}} \Omega_{E}^{1}(E)^{*}\right) \\
& \left(\stackrel{\mathrm{id},-i)}{\longrightarrow}\left(\mathbb{R} \otimes_{\mathbb{Q}} H_{K}^{+}\right) \otimes_{\mathbb{R}}\left(\mathbb{R} \otimes_{\mathbb{Q}} \Omega_{E}^{1}(E)^{*}\right)\right. \\
& \oplus\left(\mathbb{R} \otimes_{\mathbb{Q}} H_{K}^{-}\right) \otimes_{\mathbb{R}}\left(\mathbb{R} \otimes_{\mathbb{Q}} \Omega_{E}^{1}(E)^{*}\right) \\
= & \left(\mathbb{R} \otimes_{\mathbb{Q}} H_{K}\right) \otimes_{\mathbb{R}}\left(\mathbb{R} \otimes_{\mathbb{Q}} \Omega_{E}^{1}(E)^{*}\right) \\
& \stackrel{\tilde{\rho}_{K}^{-1} \otimes_{\mathrm{id}}^{\longrightarrow}}{\longrightarrow}\left(\mathbb{R} \otimes_{\mathbb{Q}} K\right) \otimes_{\mathbb{R}}\left(\mathbb{R} \otimes_{\mathbb{Q}} \Omega_{E}^{1}(E)^{*}\right) \\
= & \mathbb{R} \otimes_{\mathbb{Q}} K \otimes_{\mathbb{Q}} \Omega_{E}^{1}(E)^{*} .
\end{aligned}
$$

Proposition 3.1. Fix $\iota \in \Sigma(K)$ and define $\tau \in G$ by $c \circ \iota=$ $\iota \circ \tau$. Let $\alpha_{0} \in K$ be a normal basis element.

(a) The elements

$$
\frac{1+c}{2} w_{\iota} \otimes \gamma_{+}^{*}+\frac{1-c}{2} w_{\iota} \otimes \gamma_{-}^{*}
$$

and $\alpha_{0} \otimes \omega_{0}^{*}$ are $\mathbb{Q}[G]$-bases of $\left[H_{K} \otimes_{\mathbb{Q}} \mathcal{H}\right]^{+}$and $K \otimes_{\mathbb{Q}} \Omega_{E}^{1}(E)^{*}$, respectively.

(b) With respect to these bases, the period isomorphism $\pi_{K}$ is represented by

$$
\begin{aligned}
\lambda_{\alpha_{0}}:= & \left(\Omega_{+} \frac{1+\tau}{2}+\Omega_{-} \frac{1-\tau}{2}\right) \\
& \times\left(\sum_{\sigma \in G}(\iota \circ \sigma)\left(\alpha_{0}\right) \sigma^{-1}\right)^{-1} .
\end{aligned}
$$

Proof. Statement (a) is obvious. For the proof of (b), we write $\pi_{K}=\left(\tilde{\rho}_{K}^{-1} \otimes \mathrm{id}\right) \circ f$ and first compute the matrix for

$$
\begin{aligned}
f: & \mathbb{R} \otimes_{\mathbb{Q}}\left[H_{K} \otimes_{\mathbb{Q}} \mathcal{H}\right]^{+} \\
& \longrightarrow\left(\mathbb{R} \otimes_{\mathbb{Q}} H_{K}\right) \otimes_{\mathbb{R}}\left(\mathbb{R} \otimes_{\mathbb{Q}} \Omega_{E}^{1}(E)^{*}\right)
\end{aligned}
$$

with respect to the bases

$$
W:=1 \otimes \frac{1+c}{2} w_{\iota} \otimes \gamma_{+}^{*}+1 \otimes \frac{1-c}{2} w_{\iota} \otimes \gamma_{-}^{*}
$$

and $\left(1 \otimes w_{\iota}\right) \otimes\left(1 \otimes \omega_{0}^{*}\right)$.

The basis element $W$ is mapped to

$$
\left(\Omega_{+} \otimes \frac{1+c}{2} w_{\iota}-i \Omega_{-} \otimes \frac{1-c}{2} w_{\iota}\right) \otimes\left(1 \otimes \omega_{0}^{*}\right) .
$$

By the definition of $\tau$, one has

$$
\begin{aligned}
& \left(\Omega_{+} \frac{1+\tau}{2}-i \Omega_{-} \frac{1-\tau}{2}\right)\left(1 \otimes w_{\iota}\right) \\
& \quad=\left(\Omega_{+} \otimes \frac{1+c}{2} w_{\iota}-i \Omega_{-} \otimes \frac{1-c}{2} w_{\iota}\right) .
\end{aligned}
$$

Next we compute the matrix of the map $\tilde{\rho}_{K}:\left(\mathbb{R} \otimes_{Q}\right.$ $K) \longrightarrow \mathbb{R} \otimes_{Q} H_{K}$ with respect to the bases $1 \otimes \alpha_{0}$ and $1 \otimes w_{\iota}$. One has

$$
\tilde{\rho}_{K}\left(1 \otimes \alpha_{0}\right)=\left(\operatorname { R e } \left(\iota\left(\sigma\left(\alpha_{0}\right)\right)+\operatorname{Im}\left(\iota\left(\sigma\left(\alpha_{0}\right)\right)\right)_{\sigma \in G} .\right.\right.
$$

On the other hand, one computes

$$
\begin{gathered}
\left(\sum_{\sigma \in G} \iota\left(\sigma\left(\alpha_{0}\right)\right) \sigma^{-1}\right)\left(\frac{1+\tau}{2}-i \frac{1-\tau}{2}\right)\left(1 \otimes w_{\iota}\right) \\
=\left(\operatorname{Re}\left(\iota\left(\sigma\left(\alpha_{0}\right)\right)\right)+\operatorname{Im}\left(\iota\left(\sigma\left(\alpha_{0}\right)\right)\right)\right)_{\sigma \in G} .
\end{gathered}
$$

Summarizing, we obtain

$$
\begin{aligned}
& \pi_{K}(W)=\left(\left(\tilde{\rho}_{K}^{-1} \otimes \mathrm{id}\right) \circ f\right)(W) \\
&=\left(\Omega_{+} \frac{1+\tau}{2}-i \Omega_{-} \frac{1-\tau}{2}\right) \\
& \times\left(\tilde{\rho}_{K}^{-1} \otimes \mathrm{id}\right)\left(\left(1 \otimes w_{\iota}\right) \otimes\left(1 \otimes \omega_{0}^{*}\right)\right) \\
&=\left(\Omega_{+} \frac{1+\tau}{2}-i \Omega_{-} \frac{1-\tau}{2}\right)\left(\frac{1+\tau}{2}-i \frac{1-\tau}{2}\right)^{-1} \\
& \times\left(\sum_{\sigma \in G}(\iota \circ \sigma)\left(\alpha_{0}\right) \sigma^{-1}\right)^{-1}\left(1 \otimes \alpha_{0} \otimes \omega_{0}^{*}\right) \\
&=\left(\Omega_{+} \frac{1+\tau}{2}-i \Omega_{-} \frac{1-\tau}{2}\right)\left(\frac{1+\tau}{2}+i \frac{1-\tau}{2}\right) \\
& \times\left(\sum_{\sigma \in G}(\iota \circ \sigma)\left(\alpha_{0}\right) \sigma^{-1}\right)\left(1 \otimes \alpha_{0} \otimes \omega_{0}^{*}\right) \\
&=\left(\Omega_{+} \frac{1+\tau}{2}+\Omega_{-} \frac{1-\tau}{2}\right)\left(\sum_{\sigma \in G}(\iota \circ \sigma)\left(\alpha_{0}\right) \sigma^{-1}\right)^{-1} \\
& \times\left(1 \otimes \alpha_{0} \otimes \omega_{0}^{*}\right) .
\end{aligned}
$$


For a ring $R$ and an $R$-module $W$, we set $W^{*}:=$ $\operatorname{Hom}_{R}(W, R)$ whenever there is no danger of confusion. Following [Burns and Flach 01, (29)], we define

$$
\begin{aligned}
\Xi= & \Xi\left(M_{K}\right) \\
:= & {\left[H_{f}^{1}\left(M_{K}\right)\right]_{\mathbb{Q}[G]}^{-1}\left[H_{f}^{1}\left(K, M_{K}^{*}(1)\right)^{*}\right]_{\mathbb{Q}[G]}\left[M_{B}^{+}\right]_{\mathbb{Q}[G]}^{-1} } \\
& \times\left[M_{\mathrm{dR}} / M_{\mathrm{dR}}^{0}\right]_{\mathbb{Q}[G]} \\
= & {\left.\left[E(K) \otimes_{\mathbb{Z}} \mathbb{Q}\right]_{\mathbb{Q}[G]}^{-1}\left[E(K) \otimes_{\mathbb{Z}} \mathbb{Q}\right)^{*}\right]_{\mathbb{Q}[G]}\left[M_{B}^{+}\right]_{\mathbb{Q}[G]}^{-1} } \\
& \times\left[M_{\mathrm{dR}} / M_{\mathrm{dR}}^{0}\right]_{\mathbb{Q}[G]} .
\end{aligned}
$$

The height pairing induces an $\mathbb{R}[G]$-equivariant isomorphism

$$
\delta: E(K) \otimes_{\mathbb{Z}} \mathbb{R} \longrightarrow\left(E(K) \otimes_{\mathbb{Z}} \mathbb{R}\right)^{*} .
$$

Together with the period isomorphism $\pi_{K}$, we obtain an isomorphism in $V\left(A_{\mathbb{R}}\right)=V(\mathbb{R}[G])$,

$$
\theta_{\infty}: \Xi \otimes_{\mathbb{Q}[G]} \mathbb{R}[G] \longrightarrow \mathbf{1}_{\mathbb{R}[G]} .
$$

Let $S_{\text {ram }}(K / \mathbb{Q})$ be the set of rational primes that ramify in $K / \mathbb{Q}$, and $S_{\text {bad }}(E)$ the set of rational primes where $E$ has bad reduction. We put $S:=S_{\mathrm{ram}}(K / \mathbb{Q}) \cup S_{\text {bad }}(E)$, and for a fixed prime $l$, we set $S_{l}:=S \cup\{l\}$. We let $T_{l}(E):=\lim E\left[l^{n}\right]$ denote the $l$-adic Tate module of $E$ and set $T_{l}:=\mathbb{Z}_{l}[G] \otimes_{\mathbb{Z}_{l}} T_{l}(E)$, which we regard as a (left) module over $G_{\mathbb{Q}} \times G$. Explicitly, $G_{\mathbb{Q}}$ acts diagonally, and $g(\lambda \otimes t)=\lambda g^{-1} \otimes t$ for $g \in G, \lambda \in \mathbb{Z}_{l}[G]$, and $t \in T_{l}(E)$. We further define

$$
V_{l}(E):=T_{l}(E) \otimes_{\mathbb{Z}_{l}} \mathbb{Q}_{l}, \quad V_{l}:=T_{l} \otimes_{\mathbb{Z}_{l}} \mathbb{Q}_{l} .
$$

Although it is not visible in the notation, the modules $T_{l}$ and $V_{l}$ depend on both $E$ and $K$.

We let $R \Gamma_{c}\left(\mathbb{Z}_{S_{l}}, T_{l}\right)$ denote the complex defined in [Burns and Flach 01, Sections 3.2, 3.3] and let

$$
\theta_{l}: \Xi \otimes_{A} A_{l} \longrightarrow\left[R \Gamma_{c}\left(\mathbb{Z}_{S_{l}}, T_{l}\right)\right] \otimes_{A} A_{l}
$$

be the isomorphism defined in [Burns and Flach 01, Section 3.4]. Given these data, we obtain an element

$$
R \Omega:=\iota_{\mathbb{Z}[G], \mathbb{R}}\left(\left(\left(\prod_{l}\left[R \Gamma_{c}\left(\mathbb{Z}_{S_{l}}, T_{l}\right)\right], \Xi, \prod_{l} \theta_{l}^{-1}\right), \theta_{\infty}\right)\right)
$$

in $K_{0}(\mathbb{Z}[G], \mathbb{R})$.

Next we will formulate the conjecture for which we wish to provide numerical evidence. For each character $\psi \in \operatorname{Irr}(G)$, we write $L(E / \mathbb{Q}, \psi, s)$ for the twisted HasseWeil $L$-function. We assume that $L(E / \mathbb{Q}, \psi, s)$ has an analytic continuation to all of $\mathbb{C}$ and write $L^{*}(E / \mathbb{Q}, \psi, 1)$ for the leading term in its Taylor expansion at $s=1$.

In order to compare the vector of twisted Hasse-Weil $L$-functions to the motivic $L$-function, it is necessary to recall the precise definition of the twisted Hasse-Weil $L$ functions. The $l$-adic representation attached to $E$ is

$$
H_{l}(E):=\operatorname{Hom}\left(V_{l}(E), \mathbb{Q}_{l}\right) \otimes_{\mathbb{Q}_{l}} \overline{\mathbb{Q}}_{l} .
$$

For $\chi \in \operatorname{Irr}(G)$ we write $V_{\chi}$ for a representation space for $\chi$, and without loss of generality we may regard $V_{\chi}$ as a $\overline{\mathbb{Q}}_{l}$-vector space. For primes $p \neq l$, we define local polynomials by

$$
P_{p}(E, \chi, T):=\operatorname{det}\left(1-\operatorname{Fr}_{p}^{-1} T \mid\left(H_{l}(E) \otimes_{\bar{Q}_{l}} V_{\chi}\right)^{I_{p}}\right) .
$$

As usual, we put $L_{p}(E, \chi, s):=P_{p}\left(E, \chi, p^{-s}\right)$ and $L(E / \mathbb{Q}, \chi, s):=\prod_{p} L_{p}(E, \chi, s)^{-1}$. The $l$-adic realization of $M_{K}$ is given by

$$
H_{l}\left(M_{K}\right):=\operatorname{Hom}\left(V_{l}(E), \mathbb{Q}_{l}\right)(1) \otimes_{\mathbb{Q}_{l}} H_{K, l},
$$

where we have put $H_{K, l}:=H_{K} \otimes_{\mathbb{Z}} \mathbb{Z}_{l}$. If we fix an embedding $\iota: K \hookrightarrow \overline{\mathbb{Q}}$, then $H_{l}\left(M_{K}\right)$ gets identified with $\operatorname{Hom}\left(V_{l}(E), \mathbb{Q}_{l}\right)(1) \otimes_{\mathbb{Q}_{l}} \mathbb{Q}_{l}[G]^{*}$, where $\mathbb{Q}_{l}[G]^{*}:=$ $\operatorname{Hom}\left(\mathbb{Q}_{l}[G], \mathbb{Q}_{l}\right)$ denotes the contragredient representation. By [Burns and Flach 01, Remark 7], the motivic $L$-function associated with $M_{K}$ is defined by the Euler factors

$$
\begin{aligned}
& \operatorname{Nrd}_{\mathbb{C}[G]}\left(1-\operatorname{Fr}_{p}^{-1} T \mid H_{l}\left(M_{K}\right)^{I_{p}}\right) \\
& \quad=\left(\operatorname{det}\left(1-\frac{1}{p} \operatorname{Fr}_{p}^{-1} T \mid\left(H_{l}(E) \otimes_{\bar{Q}}, V_{\bar{\chi}}\right)^{I_{p}}\right)\right)_{\chi \in \operatorname{Irr}(G)} .
\end{aligned}
$$

It easily follows that

$$
L\left(M_{K}, s\right)=(L(E / \mathbb{Q}, \bar{\chi}, s+1))_{\chi \in \operatorname{Irr}(G)} .
$$

Remark 3.2. Since $L(E / \mathbb{Q}, \bar{\chi}, s+1)$ is the complex conjugate of $L(E / \mathbb{Q}, \chi, s+1)$ for each real value $s$, it follows from Proposition 2.9 that $L^{*}\left(M_{K}\right)=$ $(L(E / \mathbb{Q}, \bar{\chi}, 1))_{\chi \in \operatorname{Irr}(G)}$ belongs to $\zeta(\mathbb{R}[G])^{\times}$.

Remark 3.3. For later reference we compute the refined Euler characteristic of the complex

$$
T_{l}^{I_{p}} \stackrel{1-\mathrm{Fr}_{p}^{-1}}{\longrightarrow} T_{l}^{I_{p}}
$$

where the nontrivial modules are placed in degrees 0 and 1 under the assumption that $T_{l}^{I_{p}}$ is $\mathbb{Z}_{l}[G]$-perfect. In this case, the refined Euler characteristic associated with the above complex is represented by $\left(L_{p}(E, \bar{\chi}, 1)\right)_{\chi \in \operatorname{Irr}(G)}$ via the middle vertical isomorphism of Figure 2. Indeed, the Weil pairing induces a $G_{\mathbb{Q}}$-equivariant isomorphism $T_{l}(E) \simeq \operatorname{Hom}\left(T_{l}(E), \mathbb{Z}_{l}\right)(1)$. Moreover, $T_{l}=$ $\mathbb{Z}_{l}[G] \otimes_{\mathbb{Z}_{l}} T_{l}(E)$ as a (left) $G$-module, so that the assertion easily follows. For the same reason, we always have

$$
\operatorname{det}\left(1-\operatorname{Fr}_{p}^{-1} \mid V_{l}^{I_{p}}\right)=\left(L_{p}(E, \bar{\chi}, 1)\right)_{\chi \in \operatorname{Irr}(G)} .
$$


We now set

$$
\mathcal{L}^{*}:=\left(L^{*}(E / \mathbb{Q}, \bar{\psi}, 1)\right)_{\psi \in \operatorname{Irr}(G)} \in \zeta(\mathbb{R}[G])^{\times},
$$

so that $L^{*}\left(M_{K}\right)=\mathcal{L}^{*}$. If we define

$$
T \Omega:=R \Omega+\delta\left(\mathcal{L}^{*}\right)
$$

then the ETNC (see [Burns and Flach 01, Conjecture 4(iv)]) for the pair $\left(M_{K}, \mathbb{Z}[G]\right)$ can be stated in the following form.

\section{Conjecture 3.4.}

$$
T \Omega=0 \text { is in } K_{0}(\mathbb{Z}[G], \mathbb{R}) .
$$

For a set of places $P$ of $\mathbb{Q}$, we write $P(K)$ for the set of places of $K$ lying above places in $P$. In the next section we will (assuming the rationality conjecture [Burns and Flach 01, Conjecture 4(iii)] and certain further hypotheses on $K, E$, and $l$ ) describe the $l$-part $T \Omega_{l} \in K_{0}\left(\mathbb{Z}_{l}[G], \mathbb{Q}_{l}\right)$ of $T \Omega$ in terms of refined Euler characteristics. To that end, we will define a $\mathbb{Z}_{l}[G]$-perfect complex $R \Gamma_{f}\left(\mathbb{Q}, T_{l}\right)$, and for each $v \in S_{\infty}(K) \cup S_{l}(K)$ a $\mathbb{Z}_{l}[G]$-perfect complex $R \Gamma_{f}\left(K_{v}, T_{l}(E)\right)$ such that there is an exact triangle

$$
\begin{aligned}
& \bigoplus_{v \in S_{\infty}(K) \cup S_{l}(K)} R \Gamma_{f}\left(K_{v}, T_{l}(E)\right)[-1] \longrightarrow R \Gamma_{c}\left(\mathbb{Z}_{S_{l}}, T_{l}\right) \\
& \quad R \Gamma_{f}\left(K, T_{l}(E)\right) .
\end{aligned}
$$

This may be considered an explicit integral version of the middle column of [Burns and Flach 01, diagram (26)].

We will now use the additivity of refined Euler characteristics (see [Breuning and Burns 05, Theorem 5.7] and our Remark 2.3) and the explicit nature of the complexes $R \Gamma_{f}\left(K, T_{l}(E)\right)$ and $R \Gamma_{f}\left(K_{v}, T_{l}(E)\right)$ to describe $R \Omega$. We write $\chi_{\mathbb{Z}_{l}[G], \mathbb{C}_{l}}$ for the refined Euler characteristic introduced in [Burns 04]. In this way, we obtain

$$
\begin{aligned}
R \Omega_{l}= & \chi_{\mathbb{Z}_{l}[G], \mathbb{C}_{l}} \\
\times & \left(\bigoplus_{v \in S_{\infty}(K) \cup S_{l}(K)} R \Gamma_{f}\left(K_{v}, T_{l}(E)\right)[-1], \pi_{K}^{-1}\right) \\
& +\chi_{\mathbb{Z}_{l}[G], \mathbb{C}_{l}}\left(R \Gamma_{f}\left(K, T_{l}(E)\right), \delta^{-1}\right) .
\end{aligned}
$$

To conclude this section we aim to formulate an explicit rationality conjecture. As we will see, one has

$$
\begin{aligned}
H_{f}^{i}\left(K, V_{l}(E)\right) & =H_{f}^{i}\left(K, T_{l}(E)\right) \otimes_{\mathbb{Z}_{l}} \mathbb{Q}_{l} \\
& = \begin{cases}0, & i \neq 1,2, \\
E(K) \otimes_{\mathbb{Z}} \mathbb{Q}_{l}, & i=1, \\
\left(E(K) \otimes_{\mathbb{Z}} \mathbb{Q}_{l}\right)^{*}, & i=2 .\end{cases}
\end{aligned}
$$

Moreover, by [Burns and Flach 01, (28)], there is an isomorphism in $D^{\text {perf }}\left(\mathbb{Q}_{l}[G]\right)$, namely

$$
\bigoplus_{v \in S_{\infty}(K)} R \Gamma_{f}\left(K_{v}, V_{l}(E)\right) \simeq\left(M_{B}^{+} \otimes_{\mathbb{Q}} \mathbb{Q}_{l}\right)[0],
$$

and by [Burns and Flach 01, (22)] an exact triangle

$$
\begin{aligned}
& \left(\left(M_{\mathrm{dR}} / M_{\mathrm{dR}}^{0}\right) \otimes_{\mathbb{Q}} \mathbb{Q}_{p}\right)[-1] \longrightarrow \bigoplus_{v \mid l} R \Gamma_{f}\left(K_{v}, V_{l}(E)\right) \\
& \longrightarrow \bigoplus_{v \mid l}\left(V_{l, v} \stackrel{\phi_{v}}{\longrightarrow} V_{l, v}\right) .
\end{aligned}
$$

Just for the moment, we content ourselves with observing that the terms resulting from $\left(V_{l, v} \stackrel{\phi_{v}}{\longrightarrow} V_{l, v}\right)$ for $v \in S_{l}(K)$ are rational, and in fact, will give certain Euler factors. In order to state the rationality conjecture, we can therefore neglect these terms. To tie up with the situation described in Data 2.1, we set

$$
\begin{aligned}
Y^{\text {even }} & :=\left(E(K) \otimes_{\mathbb{Z}} \mathbb{Q}\right)^{*} \oplus\left(M_{\mathrm{dR}} / M_{\mathrm{dR}}^{0}\right), \\
Y^{\text {odd }} & :=\left(E(K) \otimes_{\mathbb{Z}} \mathbb{Q}\right) \oplus M_{B}^{+}, \\
\theta_{\mathbb{R}} & :=\delta^{-1} \oplus \pi_{K}^{-1}, \\
X_{l}^{\bullet} & :=R \Gamma_{f}\left(K, T_{l}(E)\right) \oplus \bigoplus_{v \mid l \infty} R \Gamma_{f}\left(K_{v}, T_{l}(E)\right)[-1] .
\end{aligned}
$$

Recall that for the rationality conjecture, we do not have to consider the maps $\theta_{l}^{\text {even }}$ and $\theta_{l}^{\text {odd }}$ (see Remark 2.7 ). Note also that it is usually more convenient to separate the height and period isomorphism and thus to consider

$$
\begin{aligned}
Y_{1}^{\text {even }} & :=\left(E(K) \otimes_{\mathbb{Z}} \mathbb{Q}\right)^{*}, \quad Y_{1}^{\text {odd }}:=E(K) \otimes_{\mathbb{Z}} \mathbb{Q}, \\
\theta_{1, \mathbb{R}} & :=\delta^{-1}, \quad X_{1, l}^{\bullet}:=R \Gamma_{f}\left(K, T_{l}(E)\right),
\end{aligned}
$$

and

$$
\begin{aligned}
Y_{2}^{\text {even }} & :=\left(M_{\mathrm{dR}} / M_{\mathrm{dR}}^{0}\right), \quad Y_{2}^{\text {odd }}:=M_{B}^{+}, \quad \theta_{2, \mathbb{R}}:=\pi_{K}^{-1}, \\
X_{2, l}^{\bullet} & :=\bigoplus_{v \mid l \infty} R \Gamma_{f}\left(K_{v}, T_{l}(E)\right)[-1] .
\end{aligned}
$$

Let $\tau_{1}$ be defined as in Remark 2.7(b) with respect to $Y_{1}^{\text {even }}$ and $Y_{1}^{\text {odd }}$. Let $\alpha_{0}$ be a normal basis element for $K / \mathbb{Q}$. For each $\chi \in \operatorname{Irr}(G)$ we choose a $\mathbb{C}$-space $V_{\chi}$ that realizes $\chi$. Let $T_{\chi}: G \longrightarrow \operatorname{Gl}\left(V_{\chi}\right)$ denote the corresponding representation, and define

$$
d_{+}(\chi):=\operatorname{dim}_{\mathbb{C}}\left(V_{\chi}^{\operatorname{Gal}(\mathbb{C} / \mathbb{R})}\right)
$$

and

$$
d_{-}(\chi):=\operatorname{codim}_{\mathbb{C}}\left(V_{\chi}^{\operatorname{Gal}(\mathbb{C} / \mathbb{R})}\right)
$$


We set

$$
\begin{aligned}
\operatorname{Reg} & =\left(\operatorname{Reg}_{\chi}\right)_{\chi \in \operatorname{Irr}(G)}:=\operatorname{Nrd}_{\mathbb{R}[G]}\left(\tau_{1}\right), \\
R & =R\left(\alpha_{0}\right)=\left(R_{\chi}\right)_{\chi \in \operatorname{Irr}(G)} \\
& :=\left(\operatorname{det}\left(\sum_{\sigma \in G} \iota\left(\sigma\left(\alpha_{0}\right)\right) T_{\chi}\left(\sigma^{-1}\right)\right)\right)_{\chi \in \operatorname{Irr}(G)}, \\
\Omega & =\left(\Omega_{\chi}\right)_{\chi \in \operatorname{Irr}(G)}:=\left(\Omega_{+}^{d_{+}(\chi)} \Omega_{-}^{d_{-}(\chi)}\right)_{\chi \in \operatorname{Irr}(G)},
\end{aligned}
$$

and note that $\operatorname{Nrd}_{\mathbb{R}[G]}\left(\lambda_{\alpha_{0}}\right)=\Omega / R\left(\alpha_{0}\right)$, where $\lambda_{\alpha_{0}}$ is defined in Proposition 3.1. From Remark 2.7(b) we deduce that the rationality part of [Burns and Flach 01, Conjecture 4$]$ is equivalent to the following conjecture.

Conjecture 3.5. We have $u \in \zeta(\mathbb{Q}[G])^{\times}$, where

$$
u:=\frac{\mathcal{L}^{*} R}{\Omega \operatorname{Reg}} \quad \text { and } \quad \mathcal{L}^{*}:=\left(L^{*}(E / \mathbb{Q}, \bar{\psi}, 1)\right)_{\psi \in \operatorname{Irr}(G)} .
$$

Remark 3.6. Conjecture 3.5 does not depend on the choice of $\alpha_{0}$. Indeed, if $\beta_{0}$ is another normal basis element, then $\beta_{0}=\lambda \alpha_{0}$ with $\lambda \in \mathbb{Q}[G]^{\times}$. It is then easy to see that $R\left(\beta_{0}\right)=\operatorname{Nrd}_{\mathbb{Q}[G]}(\lambda) R\left(\alpha_{0}\right)$.

We will compute complex approximations to

$$
u=\left(u_{\chi}\right)_{\chi \in \operatorname{Irr}(G)} \in \prod_{\chi} \mathbb{C}^{\times} \simeq \zeta(\mathbb{C}[G])^{\times}
$$

and then use Lemma 2.9 to verify the rationality conjecture numerically.

\section{EXPLICIT VERSION OF THE EQUIVARIANT TAMAGAWA NUMBER CONJECTURE}

In this section we will define the complexes $R \Gamma_{f}\left(\mathbb{Q}, T_{l}\right)$ and $R \Gamma_{f}\left(\mathbb{Q}_{p}, T_{l}\right)$ and explicitly describe their cohomology. We will closely follow [Burns and Flach 96] and [Burns 09, Section 12].

Under certain hypotheses on $E, K$, and $l$ (see below), we will derive an explicit version of ETNC in terms of refined Euler characteristics of classical objects of the theory of elliptic curves, such as the Mordell-Weil group and the Tate-Shafarevich group.

We fix an algebraic closure $\overline{\mathbb{Q}}$ of $\mathbb{Q}$ and an embed$\operatorname{ding} \iota: \overline{\mathbb{Q}} \longrightarrow \mathbb{C}$. Recall that we have defined $\tau \in G$ by $c \circ \iota=\iota \circ \tau$, with $c \in \operatorname{Gal}(\mathbb{C} / \mathbb{R})$ denoting complex conjugation. We set $G_{\infty}=\operatorname{Gal}(\mathbb{C} / \mathbb{R})$ and identify $G_{\infty}$ via $\iota$ with a subgroup of $G_{\mathbb{Q}}$. For each rational prime $p$ we fix an embedding $j_{p}: \overline{\mathbb{Q}} \longrightarrow \overline{\mathbb{Q}}_{p}$. With respect to $j_{p}$, we let $G_{p} \subseteq G_{\mathbb{Q}}$ denote the decomposition group, and $I_{p} \subseteq G_{p}$ the ramification subgroup. Finally, we let $\bar{I}_{p} \subseteq \bar{G}_{p} \subseteq G$ denote the ramification and decomposition groups of $p$ in $K / \mathbb{Q}$.

For a profinite group $\Pi$ and a continuous $\Pi$-module $N$, we denote by $C^{\bullet}(\Pi, N)$ the standard complex of continuous cochains. We write $\mathbb{Z}_{S_{l}}$ for the ring of $S_{l}$ integers, and $G_{S_{l}}$ for the Galois group of the maximal subextension of $\overline{\mathbb{Q}}$ that is unramified outside $S_{l}$. Following [Burns and Flach 01, Sections 3.2-3.4], we set

$$
R \Gamma\left(\mathbb{Z}_{S_{l}}, T_{l}\right):=C^{\bullet}\left(G_{S_{l}}, T_{l}\right)
$$

and

$$
\begin{aligned}
& R \Gamma_{c}\left(\mathbb{Z}_{S_{l}}, T_{l}\right) \\
& \quad:=\operatorname{Cone}\left(R \Gamma\left(\mathbb{Z}_{S_{l}}, T_{l}\right) \longrightarrow \bigoplus_{p \in S_{l}} C^{\bullet}\left(G_{p}, T_{l}\right)\right)[-1],
\end{aligned}
$$

where the morphism here is induced by the natural maps $G_{p} \subseteq G_{\mathbb{Q}} \longrightarrow G_{S_{l}}$.

We now proceed to define the remaining complexes in the true triangle

$$
R \Gamma_{c}\left(\mathbb{Z}_{S_{l}}, T_{l}\right) \longrightarrow R \Gamma_{f}\left(\mathbb{Q}, T_{l}\right) \longrightarrow \bigoplus_{p \in S_{l} \cup\{\infty\}} R \Gamma_{f}\left(\mathbb{Q}_{p}, T_{l}\right)
$$

Our aim is to define these complexes such that they are $\mathbb{Z}_{l}[G]$-perfect. We point out that for $l \nmid \# G$, the algebra $\mathbb{Z}_{l}[G]$ is regular, so that every complex of $\mathbb{Z}_{l}[G]$-modules with only finitely many nontrivial cohomology groups all of which are finitely generated is automatically perfect.

For a finite place $v$ of $K$ we write $\mathcal{O}_{K_{v}}$ for the valuation ring in the completion $K_{v}$, and $\mathfrak{m}_{v}$ for the maximal ideal. Let $k_{v}:=\mathcal{O}_{K_{v}} / \mathfrak{m}_{v}$ denote the residue class field. We write $E_{0}\left(K_{v}\right)$ for the points of $E\left(K_{v}\right)$ that reduce to a nonsingular point on the reduced curve $\bar{E}\left(k_{v}\right)$. Let $\bar{E}_{\mathrm{ns}}\left(k_{v}\right)$ denote the group of nonsingular points of $\bar{E}\left(k_{v}\right)$.

We will need the following set of hypotheses.

\section{Hypotheses 4.1.}

(0) $\amalg(E / K)$ is finite.

(1) $l$ is at most tamely ramified in $K / \mathbb{Q}$.

(2) (a) If $l \in S$ or $l=2$, then $l \nmid \# G$.

(b) If $l \notin S$ and $l \neq 2$, then $l \nmid \bar{I}_{p}$ for all $p \in S$.

(3) $S_{\text {bad }}(E) \cap S_{\text {ram }}(K / \mathbb{Q})=\emptyset$.

(4) If $l \mid \# G$, then

(a) $E(K) \otimes_{\mathbb{Z}} \mathbb{Z}_{l},\left(E(K) \otimes_{\mathbb{Z}} \mathbb{Z}_{l}\right)^{*}$ are $\mathbb{Z}_{l}[G]$-perfect and $l \nmid \# E(K)_{\text {tors }}$.

(b) $l \nmid \# \amalg(E / K)$.

(5) If $l \notin S$ and $l \neq 2$, then $l \nmid \#\left(E\left(K_{v}\right) / E_{0}\left(K_{v}\right)\right)$ for all $v \in S(K)$. 
Hypotheses 4.1(2) and (3) are needed to show that the above complexes are perfect. Hypotheses 4.1(1), (4), and (5) will be needed to compute the refined Euler characteristics of these complexes. Note that Hypothesis 4.1(2) possibly excludes certain prime divisors $l$ of \# $G$ from our considerations. However, in certain circumstances, all of the hypotheses (1)-(5) are conjecturally satisfied for some divisors $l$ of $\# G$. Explicit examples are given in Section 6. We remark also that with some additional effort it would be possible to relax Hypothesis 4.1(5) by stipulating it only for $l \mid \# G$, but for a related, more complicated, module. However, in this case the computation of the relevant Euler characteristics becomes more complicated and less suitable for numerical computations (see Remark 4.4).

If $p=\infty$, we define $R \Gamma_{f}\left(\mathbb{Q}_{p}, T_{l}\right)$ to be the complex $H^{0}\left(\mathbb{Q}_{\infty}, T_{l}\right)[0]=T_{l}^{G_{\infty}}[0]$. Then $R \Gamma_{f}\left(\mathbb{Q}_{\infty}, T_{l}\right)$ is indeed a perfect complex. This is clear for $l \nmid \# G$ by the preceding remark. In general, we may identify $E(\mathbb{C})$ with the complex torus $\mathbb{C} /\left(\mathbb{Z} \Omega_{+} \oplus \mathbb{Z} \Omega_{-}\right)$. In this way, we obtain an isomorphism of $G_{\infty}$-modules $T_{l}(E) \simeq \mathbb{Z}_{l} \Omega_{+} \oplus \mathbb{Z}_{l} \Omega_{-}$. Using this identification, it is clear that

$$
T_{l}(E)^{G_{v}} \simeq \begin{cases}\mathbb{Z}_{l} \Omega_{+}, & \text {if } v \text { is real, } \\ \mathbb{Z}_{l} \Omega_{+} \oplus \mathbb{Z}_{l} \Omega_{-}, & \text {if } v \text { is complex, }\end{cases}
$$

for each $v \mid \infty$. Hence $R \Gamma_{f}\left(\mathbb{Q}_{\infty}, T_{l}\right)$ is free of rank 1 as a $\mathbb{Z}_{l}[G]$-module generated by $\Omega_{+}$if $K$ is totally real, and by $\frac{1+\tau}{2} \otimes \Omega_{+}+\frac{1-\tau}{2} \otimes \Omega_{-}$if $K$ is complex (note that $l \neq 2$ by Hypothesis $4.1(2 \mathrm{a})$ if $K$ is complex). For later reference, we record

$$
T_{l}^{G_{\infty}} \simeq \mathbb{Z}_{l}[G]\left(\frac{1+\tau}{2} \otimes \Omega_{+}+\frac{1-\tau}{2} \otimes \Omega_{-}\right) .
$$

For a $\mathbb{Z}$-module $A$ we write $A^{\wedge l}$ for the $l$-completion $\lim A / l^{n} A$. For each pair of primes $p$ and $l$, we write $\overleftarrow{H_{f}^{1}}\left(\mathbb{Q}_{p}, T_{l}\right)_{\mathrm{BK}}$ for the finite-support cohomology group defined in [Bloch and Kato 90, Section 3]. We will explicitly describe this group. From Kummer theory we obtain a natural monomorphism

$$
E\left(K_{v}\right)^{\wedge l} \longrightarrow H^{1}\left(K_{v}, T_{l}(E)\right)
$$

for each place $v \mid p$. By [Bloch and Kato 90, following (3.2)], the group $H_{f}^{1}\left(K_{v}, T_{l}(E)\right)_{\mathrm{BK}}$ is equal to the image of $E\left(K_{v}\right)$ in $H^{1}\left(K_{v}, T_{l}(E)\right)$ under the composite map

$$
E\left(K_{v}\right) \longrightarrow E\left(K_{v}\right)^{\wedge l} \longrightarrow H^{1}\left(K_{v}, T_{l}(E)\right) .
$$

Using (4-8), one can show that $E\left(K_{v}\right) \longrightarrow E\left(K_{v}\right)^{\wedge l}$ is onto, so that

$$
H_{f}^{1}\left(\mathbb{Q}_{p}, T_{l}\right)_{\mathrm{BK}} \simeq \bigoplus_{v \mid p} E\left(K_{v}\right)^{\wedge l} .
$$

The next definition is motivated by [Burns 09, Section 12.2 and Remark 12.4.2]. We need the following notation. If $p \notin S$ and $p \neq 2$, then we define a finitely generated $\mathbb{Z}_{p}[G]$-module by setting $\mathcal{D}_{p}:=D_{c r, p}\left(T_{p}\right) \simeq$ $\mathcal{O}_{K, p} \otimes_{\mathbb{Z}_{p}} D_{c r, p}\left(T_{p}(E)\right)$, where $\mathcal{O}_{K, p}:=\mathbb{Z}_{p} \otimes_{\mathbb{Z}} \mathcal{O}_{K}$ and $D_{c r, p}$ is the quasi-inverse to the functor of Fontaine and Lafaille that is used in [Niziol 93]. For each such $p$ we also write $\phi_{p}$ for the natural $\mathbb{Z}_{p}[G]$-equivariant Frobenius on $\mathcal{D}_{p}$.

We define

$R \Gamma_{f}\left(\mathbb{Q}_{p}, T_{l}\right)= \begin{cases}T_{l}^{I_{p}} \stackrel{1-\mathrm{Fr}_{p}^{-1}}{\longrightarrow} T_{l}^{I_{p}}, & \text { if } l \notin S, l \neq 2, l \neq p, \\ F^{0} \mathcal{D}_{p} \stackrel{1-\phi_{p}^{0}}{\longrightarrow} \mathcal{D}_{p}, & \text { if } l \notin S, l \neq 2, l=p, \\ H_{f}^{1}\left(\mathbb{Q}_{p}, T_{l}\right)_{\mathrm{BK}}, & \text { if } l \in S \text { or } l=2,\end{cases}$

where $\operatorname{Fr}_{p}$ is the natural Frobenius in $\operatorname{Gal}\left(\mathbb{Q}_{p}^{\text {ur }} / \mathbb{Q}_{p}\right)$, and $\phi_{p}^{0}$ is the restriction of $\phi_{p}$ to $F^{0} \mathcal{D}_{p} \subseteq \mathcal{D}_{p}$. In the first two cases, the modules are placed in degrees 0 and 1 , while in the third case, the module is placed in degree 1 .

The following lemma and its proof are analogous to [Burns 09, Lemma 12.2.1].

Lemma 4.2. Assume Hypotheses 4.1(2) and (3). Then $R \Gamma_{f}\left(\mathbb{Q}_{p}, T_{l}\right)$ is a perfect complex of $\mathbb{Z}_{l}[G]$-modules.

Proof. If $l \in S$ or $l=2$, the result is clear, because in this case, $\mathbb{Z}_{l}[G]$ is regular. If $l \notin S, l \neq 2$, and $l=p$, we note that $p$ is unramified in $K / \mathbb{Q}$ by definition of $S$, and hence $\mathcal{O}_{K, p}$ is a free $\mathbb{Z}_{p}[G]$-module. Thus

$$
F^{0} \mathcal{D}_{p} \simeq \mathcal{O}_{K, p} \otimes_{\mathbb{Z}_{p}} F^{0} D_{c r, p}\left(T_{p}(E)\right)
$$

and

$$
\mathcal{D}_{p} \simeq \mathcal{O}_{K, p} \otimes_{\mathbb{Z}_{p}} D_{c r, p}\left(T_{p}(E)\right)
$$

are finitely generated $\mathbb{Z}_{p}[G]$-modules of finite projective dimension.

Finally, if $l \notin S, l \neq 2$, and $l \neq p$, we first note that

$$
T_{l}^{I_{p}}=\left(\mathbb{Z}_{l}[G] \otimes_{\mathbb{Z}_{l}} T_{l}(E)\right)^{I_{p}}=\mathbb{Z}_{l}[G]^{I_{p}} \otimes_{\mathbb{Z}_{l}} T_{l}(E)^{I_{p}},
$$

because of Hypothesis 4.1(3). If $p \in S_{\mathrm{ram}}(K / \mathbb{Q})$, then $T_{l}^{I_{p}}=\mathbb{Z}_{l}[G]^{\bar{I}_{p}} \otimes_{\mathbb{Z}_{l}} T_{l}(E)$. We write $e_{\bar{I}_{p}}$ for the idempotent associated with $\bar{I}_{p}$. By Hypothesis $4.1(2 \mathrm{~b})$, we have

$$
\begin{aligned}
\mathbb{Z}_{l}[G] & =\mathbb{Z}_{l}[G] e_{\bar{I}_{p}} \oplus \mathbb{Z}_{l}[G]\left(1-e_{\bar{I}_{p}}\right) \\
& =\mathbb{Z}_{l}[G]^{\bar{I}_{p}} \oplus \mathbb{Z}_{l}[G]\left(1-e_{\bar{I}_{p}}\right) .
\end{aligned}
$$

Therefore $T_{l}^{I_{p}}$ is a direct summand of $\mathbb{Z}_{l}[G] \otimes_{\mathbb{Z}_{l}} T_{l}(E) \simeq$ $\mathbb{Z}_{l}[G]^{2}$ and thus projective.

If $p \in S_{\text {bad }}(E)$, then $T_{l}^{I_{p}}=\mathbb{Z}_{l}[G] \otimes_{\mathbb{Z}_{l}} T_{l}(E)^{I_{p}}$ is clearly $\mathbb{Z}_{l}[G]$-free. 
We define the complex $R \Gamma\left(\mathbb{Q}, T_{l}\right)$ as in [Burns and Flach 96, (1.33)] and proceed to recall the computation of its cohomology (for more details see [Burns and Flach 96, Section 1.5.1]). For an arbitrary $\mathbb{Z}_{l}$-module $W$, we write $W^{*}$ for the linear dual $\operatorname{Hom}_{\mathbb{Z}_{l}}\left(W, \mathbb{Z}_{l}\right)$, and $W^{\vee}$ for the Pontryagin dual $\operatorname{Hom}_{\text {cont }}\left(W, \mathbb{Q}_{l} / \mathbb{Z}_{l}\right)$.

We note that the Weil pairing induces an isomorphism between $T_{l}$ and $T_{l}^{*}(1)$. Furthermore, we recall that

$$
H^{i}\left(\mathbb{Z}_{S_{l}}, T_{l}\right) \simeq \begin{cases}0, & \text { if } i=0 \\ E(K) \otimes_{\mathbb{Z}} \mathbb{Z}_{l}, & \text { if } i=1 .\end{cases}
$$

\subsection{The case $I \notin S$ and $I \neq 2$}

For a finite $\mathbb{Z}$-module $C$ we write $C_{l^{\infty}}$ for the $l$-Sylow subgroup of $C$. From [Burns and Flach 96, (1.35)-(1.37)] we derive

$$
\begin{aligned}
H_{f}^{0}\left(\mathbb{Q}, T_{l}\right) & =H^{0}\left(\mathbb{Z}_{S_{l}}, T_{l}\right)=0, \\
H_{f}^{3}\left(\mathbb{Q}, T_{l}\right) & =\left(H^{1}\left(\mathbb{Z}_{S_{l}}, T_{l}^{*}(1)\right)_{\text {tors }}\right)^{\vee} \\
& \simeq\left(\left(E(K) \otimes_{\mathbb{Z}} \mathbb{Z}_{l}\right)_{\text {tors }}\right)^{\vee}, \\
H_{f}^{i}\left(\mathbb{Q}, T_{l}\right) & =0 \text { for } i \geq 4 .
\end{aligned}
$$

Defining $\amalg\left(T_{l}^{*}(1)\right)$ as in [Burns and Flach 96], we have the short exact sequence (see [Burns and Flach 96, (1.36)])

$$
\begin{aligned}
0 \longrightarrow \amalg\left(T_{l}^{*}(1)\right)^{\vee} & \longrightarrow H_{f}^{2}\left(\mathbb{Q}, T_{l}\right) \\
& \longrightarrow H_{f}^{1}\left(\mathbb{Q}, T_{l}^{*}(1)\right)^{*} \longrightarrow 0 .
\end{aligned}
$$

By this sequence we identify $H_{f}^{2}\left(\mathbb{Q}, T_{l}\right)_{\text {tors }}$ with Ш $\left(T_{l}^{*}(1)\right)^{\vee}$, and $H_{f}^{2}\left(\mathbb{Q}, T_{l}\right)_{\mathrm{tf}}$ with $H_{f}^{1}\left(\mathbb{Q}, T_{l}^{*}(1)\right)^{*}$.

We let

$$
C\left(\mathbb{Q}_{p}, T_{l}\right) \simeq H^{0}\left(\mathbb{Q}_{p}, H^{1}\left(I_{p}, T_{l}\right)_{\text {tors }}\right)
$$

be the module introduced in [Burns and Flach 96, (1.38)], so that we have exact sequences (by [Burns and Flach 96, (1.38)] and the displayed exact sequence succeeding it)

$$
\begin{aligned}
0 \longrightarrow H_{f}^{1}\left(\mathbb{Q}_{p}, T_{l}\right) & \longrightarrow H_{f}^{1}\left(\mathbb{Q}_{p}, T_{l}\right)_{\mathrm{BK}} \\
& \longrightarrow C\left(\mathbb{Q}_{p}, T_{l}\right) \longrightarrow 0
\end{aligned}
$$

and

$$
\begin{aligned}
0 \longrightarrow H_{f}^{1}\left(\mathbb{Q}, T_{l}\right) \longrightarrow H_{f}^{1}\left(\mathbb{Q}, T_{l}\right)_{\mathrm{BK}} \longrightarrow \mathcal{C} \\
\quad \longrightarrow \amalg\left(T_{l}^{*}(1)\right)^{\vee} \longrightarrow \amalg\left(T_{l}^{*}(1)\right)_{\mathrm{BK}}^{\vee} \longrightarrow 0,
\end{aligned}
$$

with

$$
\mathcal{C}:=\bigoplus_{p \in S_{l}} C\left(\mathbb{Q}_{p}, T_{l}\right)
$$

We claim that under our assumptions, the module $\mathcal{C}$ is trivial. If $p=l$, then $H^{1}\left(I_{l}, T_{l}\right)=\operatorname{Hom}_{\text {cont }}\left(I_{l}, T_{l}\right)$, because $I_{l}$ acts trivially on $T_{l}$ (recall that $l \notin S$ ). Therefore $H^{1}\left(I_{l}, T_{l}\right)$ is torsion-free, and $C\left(\mathbb{Q}_{l}, T_{l}\right)$ is trivial. Assume now that $p \neq l$. We fix a place $v$ of $K$ above $p$ and set $L:=K_{v} \mathbb{Q}_{p}^{\text {ur }}$, where $\mathbb{Q}_{p}^{\text {ur }}$ denotes the maximal unramified extension of $\mathbb{Q}_{p}$. Furthermore, we put $U:=\operatorname{Gal}\left(\overline{\mathbb{Q}}_{p} / L\right)$. From the inflation-restriction sequence we derive

$$
\begin{aligned}
0 \longrightarrow H^{1}\left(\bar{I}_{p}, T_{l}^{U}\right) & \longrightarrow H^{1}\left(I_{p}, T_{l}\right) \\
& \longrightarrow H^{1}\left(U, T_{l}\right)^{\bar{I}_{p}} \longrightarrow H^{2}\left(\bar{I}_{p}, T_{l}^{U}\right) .
\end{aligned}
$$

If $p \in S_{\text {ram }}(K / \mathbb{Q})$, then $p \notin S_{\text {bad }}(E)$ because of Hypothesis $4.1(3)$, and we obtain $T_{l}^{U}=\mathbb{Z}_{l}[G] \otimes_{\mathbb{Z}_{l}} T_{l}(E)$, which is a cohomologically trivial $\bar{I}_{p}$-module. Hence

$$
H^{1}\left(I_{p}, T_{l}\right) \simeq H^{1}\left(U, T_{l}\right)^{\bar{I}_{p}} \simeq \operatorname{Hom}_{\mathrm{cont}}\left(U, T_{l}\right)^{\bar{I}_{p}},
$$

where the second isomorphism holds because $U$ acts trivially on $T_{l}$. It follows that $H^{1}\left(I_{p}, T_{l}\right)$ is torsionfree, which, in turn, proves the claim for primes $p \in$ $S_{\text {ram }}(K / \mathbb{Q})$.

For $p \notin S_{\text {ram }}(K / \mathbb{Q})$ we have $\bar{I}_{p}=1$, so that we get $H^{1}\left(I_{p}, T_{l}\right) \simeq H^{1}\left(U, T_{l}\right)$. Now $U=I_{p}$ acts trivially on $\mathbb{Z}_{l}[G]$, and we obtain

$$
H^{1}\left(I_{p}, T_{l}\right) \simeq \mathbb{Z}_{l}[G] \otimes_{\mathbb{Z}_{l}} H^{1}\left(U, T_{l}(E)\right) .
$$

It follows that

$$
\begin{aligned}
H^{1} & \left(I_{p}, T_{l}\right)_{\mathrm{tors}}^{G_{p}} \\
& \simeq\left(\mathbb{Z}_{l}[G] \otimes_{\mathbb{Z}_{l}} H^{1}\left(I_{p}, T_{l}(E)\right)_{\mathrm{tors}}\right)^{G_{p}} \\
& \simeq \mathbb{Z}_{l}[G] \otimes_{\mathbb{Z}_{l}\left[\bar{G}_{p}\right]} H^{1}\left(I_{p}, T_{l}(E)\right)_{\text {tors }}^{\operatorname{Gal}\left(\overline{\mathbb{Q}}_{p} / K_{v}\right)} .
\end{aligned}
$$

By [Grothendieck 72, Exposé IX, (11.3.8)], the group $H^{1}\left(I_{p}, T_{l}(E)\right)_{\mathrm{tors}}^{\mathrm{Gal}\left(\overline{\mathbb{Q}}_{p} / K_{v}\right)}$ can be identified with the $l$ primary part of $E\left(K_{v}\right) / E_{0}\left(K_{v}\right)$, and the claim follows now from Hypothesis 4.1(5).

From (4-3) and (4-4), we now deduce

$$
\begin{aligned}
H_{f}^{1}\left(\mathbb{Q}_{p}, T_{l}\right) & \simeq H_{f}^{1}\left(\mathbb{Q}_{p}, T_{l}\right)_{\mathrm{BK}}, \\
H_{f}^{1}\left(\mathbb{Q}, T_{l}\right) & \simeq H_{f}^{1}\left(\mathbb{Q}, T_{l}\right)_{\mathrm{BK}}, \\
\amalg\left(T_{l}^{*}(1)\right) & \simeq \amalg\left(T_{l}^{*}(1)\right)_{\mathrm{BK}} .
\end{aligned}
$$

By [Burns and Flach 96, (1.39)], we may identify $\amalg\left(T_{l}^{*}(1)\right)_{\mathrm{BK}}^{\vee}$ with $\amalg\left(T_{l}\right)_{\mathrm{BK}}$, which in turn identifies with $\amalg(E / K) \otimes_{\mathbb{Z}} \mathbb{Z}_{l}$.

We recall from [Bloch and Kato 90, Proposition 5.4] that $H_{f}^{1}\left(\mathbb{Q}, T_{l}\right)_{\mathrm{BK}} \simeq E(K) \otimes_{\mathbb{Z}} \mathbb{Z}_{l}$, so that

$$
\begin{aligned}
H_{f}^{1}\left(\mathbb{Q}, T_{l}\right) & \simeq E(K) \otimes_{\mathbb{Z}} \mathbb{Z}_{l}, \\
H_{f}^{2}\left(\mathbb{Q}, T_{l}\right)_{\mathrm{tf}} & \simeq\left(E(K) \otimes_{\mathbb{Z}} \mathbb{Z}_{l}\right)^{*} .
\end{aligned}
$$

Our next aim is to compute the refined Euler characteristic $\chi_{\mathbb{Z}_{l}[G], \mathbb{C}_{l}}\left(R \Gamma_{f}\left(\mathbb{Q}, T_{l}\right), \delta^{-1}\right)$ introduced in $(3-2)$ 
in terms of classical modules of the theory of elliptic curves. In full generality, this is a very difficult task, because it seems to be very hard to compute the complex $R \Gamma_{f}\left(\mathbb{Q}, T_{l}\right)$. Our hypotheses allow us to use [Burns and Flach 01, Proposition 2.1 (4)], so that we can work entirely with the cohomology modules.

Lemma 4.3. Assume Hypotheses 4.1 and write $\chi=$ $\chi_{\mathbb{Z}_{l}[G], \mathbb{C}_{l}}$. Then

$$
\begin{aligned}
\chi( & \left.R \Gamma_{f}\left(\mathbb{Q}, T_{l}\right), \delta^{-1}\right) \\
= & {\left[\left(E(K) \otimes_{\mathbb{Z}} \mathbb{Z}_{l}\right)^{*}, \delta^{-1}, E(K)_{\mathrm{tf}} \otimes_{\mathbb{Z}} \mathbb{Z}_{l}\right] } \\
& -\chi\left(\amalg(E / K)_{l^{\infty}}, 0\right)+\chi\left(E(K)_{l^{\infty}}, 0\right) \\
& +\chi\left(E(K)_{l^{\infty}}^{\vee}, 0\right) .
\end{aligned}
$$

Proof. The proof follows from the preceding computation of cohomology and Lemma 2.4.

Remark 4.4. If we relax Hypothesis 4.1(5), the module $\mathcal{C}$ is possibly nontrivial. Combining the exact sequences (4-2) and (4-4), we derive

$$
\begin{aligned}
0 & \longrightarrow H_{f}^{1}\left(\mathbb{Q}, T_{l}\right) \longrightarrow H_{f}^{1}\left(\mathbb{Q}, T_{l}\right)_{\mathrm{BK}} \\
& \longrightarrow \mathcal{C} \longrightarrow H_{f}^{2}\left(\mathbb{Q}, T_{l}\right) \longrightarrow \mathcal{S} \longrightarrow 0,
\end{aligned}
$$

with a module $\mathcal{S}$ that sits in a short exact sequence of the form

$$
0 \longrightarrow \amalg(E / K) \otimes_{\mathbb{Z}} \mathbb{Z}_{l} \longrightarrow \mathcal{S} \longrightarrow H_{f}^{1}\left(\mathbb{Q}, T_{l}\right)^{*} \longrightarrow 0 .
$$

The module $\mathcal{S}$ is related to the integral selmer group defined in [Mazur and Tate 87] (see [Burns 09, Lemma 12.2.2] and its proof). It is certainly possible to compute $\chi\left(R \Gamma_{f}\left(\mathbb{Q}, T_{l}\right), \delta^{-1}\right)$ in this more general setting for $l \nmid \# G$. However, any description of $\chi\left(R \Gamma_{f}\left(\mathbb{Q}, T_{l}\right), \delta^{-1}\right)$ would then involve the modules $\mathcal{S}$, $\mathcal{C}$, and $\amalg\left(T_{l}^{*}(1)\right)$. For computational purposes this seems to be less useful.

We now compute $\chi\left(R \Gamma_{f}\left(\mathbb{Q}_{p}, T_{l}\right), 0\right)$ for $p \neq l, \infty$. Recall that we are still in the case $l \notin S, l \neq 2$. From the definition of $R \Gamma_{f}\left(\mathbb{Q}_{p}, T_{l}\right)$, we immediately obtain

$$
\chi\left(R \Gamma_{f}\left(\mathbb{Q}_{p}, T_{l}\right), 0\right)=\left[T_{l}^{I_{p}}, 1-\operatorname{Fr}_{p}^{-1}, T_{l}^{I_{p}}\right] .
$$

By Remark 3.3, this Euler characteristic is represented by $(L(E / \mathbb{Q}, \bar{\chi}, 1))_{\chi \in \operatorname{Irr}(G)}$.

We let $\mathcal{E}$ be a Néron model for $E$ over $\mathbb{Z}$. Because of Hypothesis $4.1(3)$, we may regard $\operatorname{Spec}\left(\mathcal{O}_{K}\right) \times_{\operatorname{Spec}(\mathbb{Z})} \mathcal{E}$ as a Néron model $\mathcal{E}_{K}$ of $E$ over $K$. Recall that we made the identification

$$
\begin{aligned}
t_{\mathrm{dR}}\left(M_{K}\right) & :=M_{\mathrm{dR}} / M_{\mathrm{dR}}^{0} \simeq H^{1}\left(E_{K}, \mathcal{O}_{E_{K}}\right) \\
& \simeq K \otimes_{\mathbb{Q}} \Omega_{E}^{1}(E)^{*} .
\end{aligned}
$$

In this way, the integral lattice $H^{1}\left(\mathcal{E}_{K}, \mathcal{O}_{\mathcal{E}_{K}}\right)$ is identified with $\mathcal{O}_{K} \otimes_{\mathbb{Z}} \Omega_{\mathcal{E}}^{1}(\mathcal{E})^{*}$. Recall that $\Omega_{\mathcal{E}}^{1}(\mathcal{E})^{*}=$ $\mathbb{Z} \omega_{0}^{*}$. We define $H_{K, \mathbb{Z}}:=\bigoplus_{\sigma \in \Sigma(K)} \mathbb{Z} \subseteq H_{K}$ and $\mathcal{H}_{\mathbb{Z}}:=$ $\operatorname{Hom}_{\mathbb{Z}}\left(H^{1}(E(\mathbb{C}), \mathbb{Z}), 2 \pi i \mathbb{Z}\right) \subseteq \mathcal{H}$. Finally, we define

$$
H_{K, \mathbb{Z}_{l}}:=\mathbb{Z}_{l} \otimes_{\mathbb{Z}} H_{K, \mathbb{Z}}
$$

and

$$
\mathcal{H}_{\mathbb{Z}_{l}}:=\mathbb{Z}_{l} \otimes_{\mathbb{Z}} \mathcal{H}_{\mathbb{Z}} .
$$

We will compare the refined Euler characteristic of

$$
R \Gamma_{f}\left(\mathbb{Q}_{\infty}, T_{l}\right) \oplus R \Gamma_{f}\left(\mathbb{Q}_{l}, T_{l}\right)
$$

with the $l$-part of

$$
\begin{aligned}
& {\left[\left(H_{K, \mathbb{Z}} \otimes_{\mathbb{Z}} \mathcal{H}_{\mathbb{Z}}\right)^{+}, \pi_{K}, H^{1}\left(\mathcal{E}_{K}, \mathcal{O}_{\mathcal{E}_{K}}\right)\right]} \\
& \quad=\left[\left(H_{K, \mathbb{Z}} \otimes_{\mathbb{Z}} \mathcal{H}_{\mathbb{Z}}\right)^{+}, \pi_{K}, \mathcal{O}_{K} \otimes_{\mathbb{Z}} \Omega_{\mathcal{E}}^{1}(\mathcal{E})^{*}\right]
\end{aligned}
$$

in $K_{0}\left(\mathbb{Z}_{l}[G], \mathbb{C}_{l}\right)$.

Using the fixed embedding $\iota: K \longrightarrow \mathbb{C}$, we identify $H_{K, \mathbb{Z}} \simeq \mathbb{Z}[G]$. It is easily shown that

$$
\begin{aligned}
& \left(H_{K, \mathbb{Z}_{l}} \otimes \mathbb{Z}_{l} \mathcal{H}_{\mathbb{Z}_{l}}\right)^{+} \\
& \quad=\mathbb{Z}_{l}[G]\left(\frac{1+\tau}{2} \otimes \gamma_{+}^{*}+\frac{1-\tau}{2} \otimes \gamma_{-}^{*}\right) .
\end{aligned}
$$

By (4-1), we may therefore identify $R \Gamma_{f}\left(\mathbb{Q}_{\infty}, T_{l}\right)=$

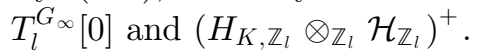

For each prime $p$, we write $t_{p}\left(V_{p}\right)$ for the tangent space $D_{\mathrm{dR}}\left(V_{p}\right) / F^{0} D_{\mathrm{dR}}\left(V_{p}\right)$ of $V_{p}$ (see [Burns and Flach 01, p. 521] for the precise definition) and

$$
\kappa_{p}: \mathbb{Q}_{p} \otimes_{\mathbb{Q}} t_{\mathrm{dR}}\left(M_{K}\right) \longrightarrow t_{p}\left(V_{p}\right)
$$

for the canonical comparison isomorphism of [Burns and Flach 01, (23)]. For any embedding $j: \mathbb{R} \longrightarrow \mathbb{C}_{l}$ we write $\pi_{K, j}$ for the composite map

$$
\begin{aligned}
\mathbb{C}_{l} \otimes_{\mathbb{R}, j} & \left.\mathbb{R} \otimes_{\mathbb{Q}}\left(H_{K} \otimes_{\mathbb{Q}} \mathcal{H}\right)^{+}\right] \\
\left.\quad \stackrel{\left(\mathbb{C}_{l} \otimes \pi_{K}\right.}{\longrightarrow}\right) & \mathbb{C}_{l} \otimes_{\mathbb{R}, j} \mathbb{R} \otimes_{\mathbb{Q}} K \otimes_{\mathbb{Q}} \Omega_{E}^{1}(E)^{*} \\
\stackrel{\left(\mathbb{C}_{l} \otimes \kappa_{l}\right)}{\longrightarrow} & \mathbb{C}_{l} \otimes_{\mathbb{Q}_{l}} t_{l}\left(V_{l}\right) .
\end{aligned}
$$

Since $l \notin S$ and $l \neq 2$, the theory of Fontaine and Messing implies that

$$
\kappa_{l}\left(\mathbb{Z}_{l} \otimes_{\mathbb{Z}} H^{1}\left(\mathcal{E}_{K}, \mathcal{O}_{\mathcal{E}_{K}}\right)\right)=\mathcal{D}_{l} / F^{0} \mathcal{D}_{l}
$$

(see the proof of [Burns 09, Lemma 12.4.1]). In particular, $\mathcal{D}_{l} / F^{0} \mathcal{D}_{l}$ is $\mathbb{Z}_{l}[G]$-projective, since

$$
\mathbb{Z}_{l} \otimes_{\mathbb{Z}} H^{1}\left(\mathcal{E}_{K}, \mathcal{O}_{\mathcal{E}_{K}}\right) \simeq \mathbb{Z}_{l} \otimes_{\mathbb{Z}} \mathcal{O}_{K} \otimes_{\mathbb{Z}} \Omega_{\mathcal{E}}^{1}(\mathcal{E})^{*}
$$


is $\mathbb{Z}_{l}[G]$-free, since $l \notin S$ is unramified in $K / \mathbb{Q}$.

Since $\mathcal{D}_{l} \stackrel{1-\phi_{l}}{\longrightarrow} \mathcal{D}_{l}$ is injective, the short exact sequence of complexes (with vertical differentials)

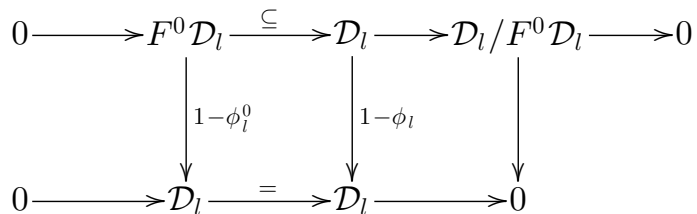

implies that

$0 \longrightarrow \mathcal{D}_{l} / F^{0} \mathcal{D}_{l} \longrightarrow H_{f}^{1}\left(\mathbb{Q}_{l}, T_{l}\right) \longrightarrow \mathcal{D}_{l} /\left(1-\phi_{l}\right) \mathcal{D}_{l} \longrightarrow 0$

is exact. It follows that $H_{f}^{1}\left(\mathbb{Q}_{l}, T_{l}\right)$ is $\mathbb{Z}_{l}[G]$-perfect and

$$
\begin{aligned}
j_{*} & \left(\left[\left(H_{K, \mathbb{Z}} \otimes_{\mathbb{Z}} \mathcal{H}_{\mathbb{Z}}\right)^{+}, \pi_{K}, \mathcal{O}_{K} \otimes_{\mathbb{Z}} \Omega_{\mathcal{E}}^{1}(\mathcal{E})^{*}\right]\right) \\
= & {\left[T_{l}^{G_{\infty}}, \pi_{K, j}, \kappa_{l}\left(\mathbb{Z}_{l} \otimes_{\mathbb{Z}} \mathcal{O}_{K} \otimes_{\mathbb{Z}} H^{1}\left(\mathcal{E}_{K}, \mathcal{O}_{\mathcal{E}_{K}}\right)\right)\right] } \\
= & {\left[T_{l}^{G_{\infty}}, \pi_{K, j}, \mathcal{D}_{l} / F^{0} \mathcal{D}_{l}\right] } \\
= & {\left[T_{l}^{G_{\infty}}, \pi_{K, j}, H_{f}^{1}\left(\mathbb{Q}_{l}, T_{l}\right)\right]-\left[\mathcal{D}_{l}, 1-\phi_{l}, \mathcal{D}_{l}\right] } \\
= & \chi_{\mathbb{Z}_{l}[G], \mathbb{C}_{l}}\left(R \Gamma_{f}\left(\mathbb{Q}_{\infty}, T_{l}\right) \oplus R \Gamma_{f}\left(\mathbb{Q}_{l}, T_{l}\right), \pi_{K}\right) \\
& -\left[\mathcal{D}_{l}, 1-\phi_{l}, \mathcal{D}_{l}\right],
\end{aligned}
$$

where the last equality follows from Lemma 2.4.

In summary, we obtain for $l \notin S, l \neq 2$,

$$
\begin{aligned}
R \Omega_{l}= & {\left[\left(E(K) \otimes_{\mathbb{Z}} \mathbb{Z}_{l}\right)^{*}, \delta^{-1}, E(K)_{\mathrm{tf}} \otimes_{\mathbb{Z}} \mathbb{Z}_{l}\right] } \\
& +\chi\left(E(K)_{l^{\infty}}, 0\right) \\
& +\chi\left(E(K)_{l^{\infty}}^{\vee}, 0\right)-\chi\left(\amalg(E / K)_{l^{\infty}}, 0\right) \\
& -\sum_{p \in S}\left[T_{l}^{I_{p}}, 1-\operatorname{Fr}_{p}^{-1}, T_{l}^{I_{p}}\right] \\
& -j_{*}\left(\left[\left(H_{K, \mathbb{Z}} \otimes_{\mathbb{Z}} \mathcal{H}_{\mathbb{Z}}\right)^{+}, \pi_{K}, \mathcal{O}_{K} \otimes_{\mathbb{Z}} \Omega_{\mathcal{E}}^{1}(\mathcal{E})^{*}\right]\right) \\
& -\left[\mathcal{D}_{l}, 1-\phi_{l}, \mathcal{D}_{l}\right] .
\end{aligned}
$$

\subsection{The case $I \in S$ or $I=2$}

By our assumptions, $l \nmid \# G$. We recall from [Burns and Flach 96, end of Section 1.5] that

$$
\begin{aligned}
& H_{f}^{i}\left(\mathbb{Q}, T_{l}\right)_{\mathrm{BK}}=0 \text { for } i \neq 1,2,3, \\
& H_{f}^{1}\left(\mathbb{Q}, T_{l}\right)_{\mathrm{BK}}=E(K) \otimes_{\mathbb{Z}} \mathbb{Z}_{l}, \\
& H_{f}^{3}\left(\mathbb{Q}, T_{l}\right)_{\mathrm{BK}}=E(K)_{l^{\infty}}^{\vee},
\end{aligned}
$$

and

$$
\begin{aligned}
0 \longrightarrow & \amalg(E / K) \otimes_{\mathbb{Z}} \mathbb{Z}_{l} \longrightarrow H_{f}^{2}\left(\mathbb{Q}, T_{l}\right)_{\mathrm{BK}} \\
& \longrightarrow\left(E(K) \otimes_{\mathbb{Z}} \mathbb{Z}_{l}\right)^{*} \longrightarrow 0 .
\end{aligned}
$$

As before, we write $\chi=\chi_{\mathbb{Z}_{l}[G], \mathbb{C}_{l}}$ for the refined Euler characteristic. If $v$ is a finite place of $K$, we also put $\chi_{v}=\chi_{\mathbb{Z}_{l}\left[G_{v}\right], \mathbb{C}_{l}}$ for the refined Euler characteristic in $K_{0}\left(\mathbb{Z}_{l}\left[G_{v}\right], \mathbb{C}_{l}\right)$. If we write

$$
\operatorname{ind}_{G_{v}}^{G}: K_{0}\left(\mathbb{Z}_{l}\left[G_{v}\right], \mathbb{C}_{l}\right) \longrightarrow K_{0}\left(\mathbb{Z}_{l}[G], \mathbb{C}_{l}\right)
$$

for the natural induction map, then $\chi=\operatorname{ind}_{G_{v}}^{G} \circ \chi_{v}$.

Applying Lemma 2.4, we obtain

$$
\begin{aligned}
\chi\left(R \Gamma_{f}\left(\mathbb{Q}, T_{l}\right), \delta^{-1}\right)= & {\left[\left(E(K) \otimes_{\mathbb{Z}} \mathbb{Z}_{l}\right)^{*}, \delta^{-1}, E(K)_{\mathrm{tf}} \otimes_{\mathbb{Z}} \mathbb{Z}_{l}\right] } \\
& +\chi\left(E(K)_{l^{\infty}}, 0\right)+\chi\left(E(K)_{l^{\infty}}^{\vee}, 0\right) \\
& -\chi\left(\amalg(E / K)_{l^{\infty}}, 0\right) .
\end{aligned}
$$

We write $\hat{E}$ for the formal group associated with $E$. Then we have the basic short exact sequence

$$
0 \longrightarrow \hat{E}\left(\mathfrak{m}_{v}\right) \longrightarrow E_{0}\left(K_{v}\right) \longrightarrow \bar{E}_{\mathrm{ns}}\left(k_{v}\right) . \longrightarrow 0 .
$$

We recall that $H_{f}^{1}\left(\mathbb{Q}_{p}, T_{l}\right) \simeq \bigoplus_{v \mid p} E\left(K_{v}\right)^{\wedge l}$. For $p \neq l$ and $v \mid p$, we first note that $\hat{E}\left(\mathfrak{m}_{v}\right)^{\wedge l}=0$. From (4-8), we derive the short exact sequence

$$
\begin{aligned}
0 \longrightarrow \bar{E}_{\mathrm{ns}}\left(k_{v}\right)_{l^{\infty}} & \longrightarrow E\left(K_{v}\right)^{\wedge l} \\
& \longrightarrow\left(E\left(K_{v}\right) / E_{0}\left(K_{v}\right)\right)_{l^{\infty}} \longrightarrow 0,
\end{aligned}
$$

so that $H_{f}^{1}\left(\mathbb{Q}_{p}, T_{l}\right)_{\mathrm{BK}}$ is finite, and by Lemma 2.4 ,

$$
\begin{aligned}
\chi\left(R \Gamma_{f}\left(\mathbb{Q}_{p}, T_{l}\right), 0\right)= & \operatorname{ind}_{G_{v}}^{G} \chi_{v}\left(\bar{E}_{\mathrm{ns}}\left(k_{v}\right)_{l^{\infty}}, 0\right) \\
& +\operatorname{ind}_{G_{v}}^{G} \chi_{v}\left(\left(E\left(K_{v}\right) / E_{0}\left(K_{v}\right)\right)_{l^{\infty}}, 0\right) .
\end{aligned}
$$

As in the previous case, we must now relate the Euler characteristic of $R \Gamma_{f}\left(\mathbb{Q}_{\infty}, T_{l}\right) \oplus R \Gamma_{f}\left(\mathbb{Q}_{l}, T_{l}\right)$ and the element $\left[\left(H_{K, \mathbb{Z}} \otimes_{\mathbb{Z}} \mathcal{H}_{\mathbb{Z}}\right)^{+}, \pi_{K}, \mathcal{O}_{K} \otimes_{\mathbb{Z}} \Omega_{\mathcal{E}}^{1}(\mathcal{E})^{*}\right]_{l}$.

We write

$$
\exp _{p}^{\mathrm{BK}}: t_{p}\left(V_{p}\right) \longrightarrow H_{f}^{1}\left(\mathbb{Q}_{p}, V_{p}\right)
$$

for the isomorphism given by the Bloch-Kato exponential map and recall that the logarithm attached to the formal group $\hat{E}$ induces an isomorphism

$$
\log _{v}: E\left(K_{v}\right)^{\wedge l} \otimes_{\mathbb{Z}_{l}} \mathbb{Q}_{l} \simeq K_{v} .
$$

We use the commutative diagram

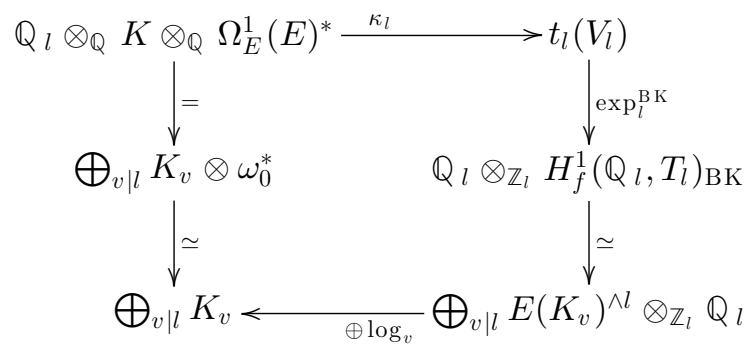

For each place $v \mid l$ we choose a positive integer $n_{v}$ such that $\log _{v}$ induces an isomorphism between $\hat{E}\left(\mathfrak{m}_{v}^{n_{v}}\right)$ and $\mathfrak{m}_{v}^{n_{v}}$. For every prime $p$, we fix a place $v_{p}$ above $p$. We obtain the result shown in Figure 3. 


$$
\begin{aligned}
j_{*} & \left.\left(\left[H_{K, \mathbb{Z}} \otimes_{\mathbb{Z}} \mathcal{H}_{\mathbb{Z}}\right)^{+}, \pi_{K}, \mathcal{O}_{K} \otimes_{\mathbb{Z}} \Omega_{\mathcal{E}}^{1}(\mathcal{E})^{*}\right]\right) \\
= & {\left[T_{l}^{G}, \pi_{K, j}, \kappa_{l}\left(\mathbb{Z}_{l} \otimes_{\mathbb{Z}} \mathcal{O}_{K} \otimes_{\mathbb{Z}} \Omega_{\mathcal{E}}^{1}(\mathcal{E})^{*}\right)\right] } \\
= & {\left[T_{l}^{G}, \pi_{K, j}, \kappa_{l}\left(\oplus_{v \mid l} \mathcal{O}_{K_{v}} \otimes \omega_{0}^{*}\right)\right] } \\
= & {\left[T_{l}^{G \infty}, \exp _{l}^{\mathrm{BK}} \circ \pi_{K, j},\left(\exp _{l}^{\mathrm{BK}} \circ \kappa_{l}\right)\left(\oplus_{v \mid l} \mathcal{O}_{K_{v}} \otimes \omega_{0}^{*}\right)\right] } \\
= & {\left[T_{l}^{G \infty}, \exp _{l}^{\mathrm{BK}} \circ \pi_{K, j},\left(\exp _{l}^{\mathrm{BK}} \circ \kappa_{l}\right)\left(\oplus_{v \mid l} \mathfrak{m}_{v}^{n_{v}} \otimes \omega_{0}^{*}\right)\right]+\left[\oplus_{v \mid l} \mathfrak{m}^{n_{v}}, \mathrm{id}, \oplus_{v \mid l} \mathcal{O}_{K_{v}}\right] } \\
\stackrel{(\mathrm{i})}{=} & {\left[T_{l}^{G \infty}, \exp _{l}^{\mathrm{BK}} \circ \pi_{K, j}, \oplus_{v \mid l} \hat{E}\left(\mathfrak{m}_{v}^{n_{v}}\right)\right]+\left[\oplus_{v \mid l} \mathfrak{m}^{n_{v}}, \mathrm{id}, \oplus_{v \mid l} \mathcal{O}_{K_{v}}\right] } \\
= & {\left[T_{l}^{G \infty}, \exp _{l}^{\mathrm{BK}} \circ \pi_{K, j}, \oplus_{v \mid l} \hat{E}\left(\mathfrak{m}_{v}\right)\right]-\left[\oplus_{v \mid l} \hat{E}\left(\mathfrak{m}^{n_{v}}\right), \mathrm{id}, \oplus_{v \mid l} \hat{E}(\mathfrak{m})\right]+\left[\oplus_{v \mid l} \mathfrak{m}^{n_{v}}, \mathrm{id}, \oplus_{v \mid l} \mathcal{O}_{K_{v}}\right] } \\
\stackrel{(i \mathrm{ii})}{=} & {\left[T_{l}^{G \infty}, \exp _{l}^{\mathrm{BK}} \circ \pi_{K, j}, \oplus_{v \mid l} E_{0}\left(K_{v}\right)^{\wedge l}\right]-\chi\left(\oplus_{v \mid l} \bar{E}_{\mathrm{ns}}\left(k_{v}\right)_{l \infty}, 0\right)+\left[\oplus_{v \mid l} \mathfrak{m}, \mathrm{id}, \oplus_{v \mid l} \mathcal{O}_{K_{v}}\right] } \\
= & {\left[T_{l}^{G}, \exp _{l}^{\mathrm{BK}} \circ \pi_{K, j}, H_{f}^{1}\left(\mathbb{Q}_{l}, T_{l}\right)_{\mathrm{BK}}\right]-\chi\left(\oplus_{v \mid l}\left(E\left(K_{v}\right) / E_{0}\left(K_{v}\right)\right)_{l \infty}, 0\right)-\chi\left(\oplus_{v \mid l} \bar{E}_{\mathrm{ns}}\left(k_{v}\right)_{l \infty}, 0\right)+\left[\oplus_{v \mid l} \mathfrak{m}, \mathrm{id}, \oplus_{v \mid l} \mathcal{O}_{K_{v}}\right] } \\
= & {\left[T_{l}^{G \infty}, \exp _{l}^{\mathrm{BK}} \circ \pi_{K, j}, H_{f}^{1}\left(\mathbb{Q}_{l}, T_{l}\right)_{\mathrm{BK}}\right]-\operatorname{ind}_{G_{v_{l}}}^{G}\left(\chi_{v_{l}}\left(\left(E\left(K_{v_{l}}\right) / E_{0}\left(K_{v_{l}}\right)\right)_{l \infty}, 0\right)\right)-\operatorname{ind}_{G_{v_{l}}}^{G}\left(\chi_{v_{l}}\left(\bar{E}_{\mathrm{ns}}\left(k_{v_{l}}\right)_{l \infty}, 0\right)\right) } \\
& +\operatorname{ind}_{G_{v_{l}}}^{G}\left(\chi_{v_{l}}\left(k_{v_{l}}, 0\right)\right) \\
& \stackrel{(\mathrm{iii})}{=} \chi\left(R \Gamma_{f}\left(\mathbb{Q}_{\infty}, T_{l}\right) \oplus R \Gamma_{f}\left(\mathbb{Q}_{l}, T_{l}\right), \pi_{K}\right)-\operatorname{ind}_{G_{v_{l}}}^{G}\left(\chi_{v_{l}}\left(\left(E\left(K_{v_{l}}\right) / E_{0}\left(K_{v_{l}}\right)\right)_{l \infty}, 0\right)\right)-\operatorname{ind}_{G_{v_{l}}}^{G}\left(\chi_{v_{l}}\left(\bar{E}_{\mathrm{ns}}\left(k_{v_{l}}\right)_{l \infty}, 0\right)\right) \\
& +\operatorname{ind}_{G_{v_{l}}}^{G}\left(\chi_{v_{l}}\left(k_{v_{l}}, 0\right)\right) .
\end{aligned}
$$

FIGURE 3. Computation of equivariant period.

Here the equality in the figure labeled (i) is induced by the diagram and our choice of integers $n_{v}$, (ii) comes from (4-8), and (iii) follows from Lemma 2.4.

In summary, we obtain for $l \in S$ or $l=2$ (always assuming $l \nmid \# G)$,

$$
\begin{aligned}
R \Omega_{l}= & {\left[\left(E(K) \otimes_{\mathbb{Z}} \mathbb{Z}_{l}\right)^{*}, \delta^{-1}, E(K)_{\mathrm{tf}} \otimes_{\mathbb{Z}} \mathbb{Z}_{l}\right] } \\
& -j_{*}\left(\left[\left(H_{K, \mathbb{Z}} \otimes_{\mathbb{Z}} \mathcal{H}_{\mathbb{Z}}\right)^{+}, \pi_{K}, \mathcal{O}_{K} \otimes_{\mathbb{Z}} \Omega_{\mathcal{E}}^{1}(\mathcal{E})^{*}\right]\right) \\
& +\chi\left(E(K)_{l^{\infty}}, 0\right)+\chi\left(E(K)_{l^{\infty}}^{\vee}, 0\right) \\
& -\chi\left(\amalg(E / K)_{l^{\infty}}, 0\right) \\
& -\sum_{p \in S_{l}} \operatorname{ind}_{G_{v_{p}}}^{G}\left(\chi_{v_{p}}\left(\bar{E}_{\mathrm{ns}}\left(k_{v_{p}}\right)_{l^{\infty}}, 0\right)\right) \\
& -\sum_{p \in S_{l}} \operatorname{ind}_{G_{v_{p}}}^{G}\left(\chi_{v_{p}}\left(\left(E\left(K_{v_{p}}\right) / E_{0}\left(K_{v_{p}}\right)\right)_{l^{\infty}}, 0\right)\right) \\
& +\operatorname{ind}_{G_{v_{l}}}^{G}\left(\chi_{v_{l}}\left(k_{v_{l}}, 0\right)\right) .
\end{aligned}
$$

If $E(K)_{\mathrm{tf}} \otimes_{\mathbb{Z}} \mathbb{Z}_{l}$ and $\left(E(K) \otimes_{\mathbb{Z}} \mathbb{Z}_{l}\right)^{*}$ are $\mathbb{Z}_{l}[G]-$ projective, then by the arguments of Remark $2.7(\mathrm{a})$, we can find a $\mathbb{Z}_{l}[G]$-module $Z$ such that both $\left(E(K)_{\mathrm{tf}} \otimes_{\mathbb{Z}}\right.$ $\left.\mathbb{Z}_{l}\right) \oplus Z$ and $\left(E(K) \otimes_{\mathbb{Z}} \mathbb{Z}_{l}\right)^{*} \oplus Z$ are $\mathbb{Z}_{l}[G]$-free. Then one has

$$
\begin{aligned}
& {\left[\left(E(K) \otimes_{\mathbb{Z}} \mathbb{Z}_{l}\right)^{*}, \delta^{-1}, E(K)_{\mathrm{tf}} \otimes_{\mathbb{Z}} \mathbb{Z}_{l}\right]} \\
& \quad=\left[\left(E(K) \otimes_{\mathbb{Z}} \mathbb{Z}_{l}\right)^{*} \oplus Z, \delta^{-1} \oplus \mathrm{id},\left(E(K)_{\mathrm{tf}} \otimes_{\mathbb{Z}} \mathbb{Z}_{l}\right) \oplus Z\right],
\end{aligned}
$$

so that without loss of generality we may assume that we can work with $\mathbb{Z}_{l}[G]$-bases.

If $T$ is a finite perfect $\mathbb{Z}_{l}[G]$-module, then we write $\tilde{\chi}_{\mathbb{Z}_{l}[G], \mathbb{C}_{l}}(T, 0)$ for any lift of $\chi_{\mathbb{Z}_{l}[G], \mathbb{C}_{l}}(T, 0)$ via the mid- dle vertical map of Figure 2. Analogously, we use the notation $\tilde{\chi}$ and $\tilde{\chi}_{v}$.

Recall the definition of $u=\frac{\mathcal{L}^{*} R}{\Omega \operatorname{Reg}}$ in Conjecture 3.5.

Proposition 4.5. Assume Hypotheses 4.1 and let $\alpha_{0}$ be a normal basis element such that $\mathcal{O}_{K, l}=\mathbb{Z}_{l}[G] \alpha_{0}$. Assume that $u=u_{l}$ is computed with respect to $\alpha_{0}$ and $a \mathbb{Z}_{l}[G]$ basis of $E(K)_{\mathrm{tf}} \otimes_{\mathbb{Z}} \mathbb{Z}_{l}$ and $\left(E(K) \otimes_{\mathbb{Z}} \mathbb{Z}_{l}\right)^{*}$. Assume also that the rationality conjecture holds.

If $l \notin S$ and $l \neq 2$, we set

$$
\begin{aligned}
\xi_{l}:=\tilde{\chi}\left(E(K)_{l^{\infty}}, 0\right)^{-1} \cdot \tilde{\chi}\left(E(K)_{l^{\infty}}^{\vee}, 0\right)^{-1} & \cdot \tilde{\chi}\left(\amalg(E / K)_{l^{\infty}}, 0\right) .
\end{aligned}
$$

If $l \in S$ or $l=2$, we set

$$
\begin{aligned}
\xi_{l}:= & \tilde{\chi}\left(E(K)_{l^{\infty}}, 0\right)^{-1} \cdot \tilde{\chi}\left(E(K)_{l^{\infty}}^{\vee}, 0\right)^{-1} \\
& \left.\cdot \tilde{\chi}(\amalg / K)_{l^{\infty}}, 0\right) \\
& \cdot \prod_{p \in S_{l}} \operatorname{ind}_{G_{v_{p}}}^{G} \tilde{\chi}_{v_{p}}\left(\bar{E}_{\mathrm{ns}}\left(k_{v_{p}}\right)_{l^{\infty}}, 0\right) \\
& \cdot \prod_{p \in S_{l}} \operatorname{ind}_{G_{v_{p}}}^{G} \tilde{\chi}_{v_{p}}\left(\left(E\left(K_{v_{p}}\right) / E_{0}\left(K_{v_{p}}\right)\right)_{l^{\infty}}, 0\right) \\
& \cdot \operatorname{ind}_{G_{v_{l}}}^{G} \tilde{\chi}_{v_{l}}\left(k_{v_{l}}, 0\right)^{-1} \prod_{p \in S_{l}}\left(L_{p}(E, \bar{\chi}, 1)\right)_{\chi \in \operatorname{Irr}_{Q}(G)}^{-1} .
\end{aligned}
$$

Then

$$
T \Omega_{l}=0 \Longleftrightarrow u_{l}=\xi_{l} \in \operatorname{cok}\left(\mu_{l}\right) \oplus I_{l}(C) .
$$

Proof. The Euler factor terms $\left[T_{l}^{I_{p}}, 1-\mathrm{Fr}_{p}^{-1}, T_{l}^{I_{p}}\right]$ and $\left[\mathcal{D}_{l}, 1-\phi_{l}, \mathcal{D}_{l}\right]$ in (4-7) cancel, because of the 
identification made in [Burns and Flach 01, (24)] applied to [Burns and Flach 01, (19) and (22)]. See Remark 3.3.

For the same reason we obtain the local Euler factors $\left(L_{p}(E, \bar{\chi}, 1)\right)_{\chi \in \operatorname{Irr}_{Q}(G)}$ in (4-9). Indeed, by (3-1), the local factors $\left(L_{p}(E, \bar{\chi}, 1)\right)_{\chi \in \operatorname{Irr}_{Q}(G)}$ represent the refined Euler characteristics of the complexes $\left(V_{p} \stackrel{\phi_{p}}{\longrightarrow} V_{p}\right)$ that occur in [Burns and Flach 01, (19) and (22)].

Remark 4.6. The local Euler factors in the above formulas can be computed using their explicit definition. For the computation of the refined Euler characteristics of finite modules we use the method explained in Example 2.8. Hence we obtain $\xi_{l}$ as an element in $C^{\times}$.

If $u_{l} \xi_{l}^{-1}=\left(\eta_{1}, \ldots, \eta_{r}\right)$ with $\eta_{i} \in K_{i}$, then $T \Omega_{l}=0$ if and only if $v_{\mathfrak{P}}\left(\eta_{i}\right)=0, \forall i \in\{1, \ldots, r\}, \mathfrak{P} \mid l \in K_{i} / \mathbb{Q}$, and $\left(\bar{\eta}_{1}, \ldots, \bar{\eta}_{r}\right) \in \operatorname{im}\left(\mu_{l}\right)$, where $\bar{\eta}_{i}$ denotes the image of $\eta_{i}$ under the projection $\mathcal{O}_{K_{i}, l}^{\times} \longrightarrow\left(\mathcal{O}_{K_{i}} / \mathfrak{g}_{i, l}\right)^{\times}$, and $\mu_{l}$ is the isomorphism from (2-4). Recall that this means that the $\eta_{i}$ have to satisfy certain complicated congruences. In Section 2.3, we made these congruences explicit for cyclic groups $Z_{l}$, dihedral groups $D_{2 l}$, and the alternating group $A_{4}$. For explicit examples see Sections 6.1, 6.2, and 6.3, where we consider dihedral extensions $K / \mathbb{Q}$ of degree $2 l$ for an odd prime $l$. In each of these examples, the prime $l$ is of particular interest, because we have to check that the BSD quotients satisfy the congruence (2-6).

Remark 4.7. The element $u_{l}$ in (4-10) depends on the choice of $\alpha_{0}$. However, the validity of the statement $u_{l}=\xi_{l} \in \operatorname{cok}\left(\mu_{l}\right) \oplus I_{l}(C)$ is independent of this choice. If $\beta_{0}$ is another $\mathbb{Z}_{l}[G]$-generator of $\mathcal{O}_{K, l}$, then $\beta_{0}=\lambda \alpha_{0}$ with a unit $\lambda \in \mathbb{Z}_{l}[G]^{\times}$. As in Remark 3.6, we see that $u_{l}\left(\beta_{0}\right)=\operatorname{Nrd}_{\mathbb{Q}_{l}[G]}(\lambda) u_{l}\left(\alpha_{0}\right)$. Hence the independence follows from the fact that $\operatorname{Nrd}_{\mathbb{Q}_{l}[G]}(\lambda)$ is a unit in $\mathcal{O}_{C, l}$ that is contained in the image of $\mu_{l}$.

We fix a normal basis element $\alpha_{0}$ and $\mathbb{Q}[G]$-bases of $E(K) \otimes_{\mathbb{Z}} \mathbb{Q}$ and $\left(E(K) \otimes_{\mathbb{Z}} \mathbb{Q}\right)^{*}$ in the sense of Remark 2.7(b) and compute $u$ with respect to these bases. Note that for almost all primes $l$, the element $\alpha_{0}$ constitutes a $\mathbb{Z}_{l}[G]$-basis of $\mathcal{O}_{K, l}$, and the chosen $\mathbb{Q}[G]$-basis of $E(K) \otimes_{\mathbb{Z}} \mathbb{Q}$, respectively $\left(E(K) \otimes_{\mathbb{Z}} \mathbb{Q}\right)^{*}$, is a $\mathbb{Z}_{l}[G]$ basis of $E(K)_{\mathrm{tf}} \otimes_{\mathbb{Z}} \mathbb{Z}_{l}$, respectively $\left(E(K) \otimes_{\mathbb{Z}} \mathbb{Z}_{l}\right)^{*}$. For all these primes $l$ we can use this fixed $u$ as $u_{l}$ in Proposition 4.5.

We define two finite sets of rational primes: The first is

$$
\mathrm{HP}_{1}=S \cup\{2\} \cup\{l: l \mid \# G\} \cup\left\{l: l \mid \# E\left(K_{v}\right) / E_{0}\left(K_{v}\right)\right.
$$

for

$$
\left.v \in S_{l}(K)\right\} \cup\left\{l: l \mid \# E(K)_{\text {tors }}\right\} \cup\{l: l \mid \# \amalg(E / K)\},
$$

and the second is

$$
\mathrm{HP}_{2}=\left\{l: u_{l} \neq u\right\} .
$$

So for all $l \notin \mathrm{HP}_{2}$ we can use the fixed $u$ as $u_{l}$ in Proposition 4.5. Note that $\mathrm{HP}_{2}$ depends on the choice of $\alpha_{0}$ and the $\mathbb{Q}[G]$-bases of $E(K) \otimes_{\mathbb{Z}} \mathbb{Q}$ and $\left(E(K) \otimes_{\mathbb{Z}} \mathbb{Q}\right)^{*}$. Finally, we set

$$
\mathrm{HP}:=\mathrm{HP}_{1} \cup \mathrm{HP}_{2} \text {. }
$$

We say that an element $w=\left(w_{1}, \ldots, w_{r}\right) \in \zeta(\mathbb{Q}[G])^{\times}$ has support in HP if $\left(w_{i}, p\right)=1$ for $i=1, \ldots, r$ and all primes $p \notin \mathrm{HP}$.

Corollary 4.8. Assume Hypotheses 4.1(0) and (3) and the rationality conjecture. Let $l \notin \mathrm{HP}$ be a rational prime. Then l satisfies Hypotheses 4.1(1), (2), (4), and (5), and

$$
T \Omega_{l}=0 \Longleftrightarrow u \text { has support in } \mathrm{HP} \text {. }
$$

Proof. The first assertion is clear from the definition of $\mathrm{HP}$. If $l \notin \mathrm{HP}$, then we are in the case $l \notin S$ and $l \neq 2$. By definition of $\mathrm{HP}_{1}$, the element $\xi_{l}$ is trivial. By definition of $\mathrm{HP}_{2}$, we have $u=u_{l}$. Since $l \nmid \# G$, we have $\operatorname{cok}\left(\mu_{l}\right)=0$, so that $T \Omega_{l}=0$ if and only if $u$ is prime to $l$ in the sense of Remark 4.6.

By the corollary we can, in principle, numerically verify ETNC for almost all primes $l$ as soon as we have computed a good approximation of $u \in \prod_{\chi \in \operatorname{Irr}(G)} \mathbb{C}^{\times}$. If the computed $u$ makes us believe that the rationality conjecture holds, and if we are able to round $u$ to an element of $u^{\prime} \in \prod_{\chi \in \operatorname{Irr}_{\mathbb{Q}}(G)} \mathbb{Q}(\chi)^{\times}$, we have only to check whether $u^{\prime}$ has support in HP.

Of course, the main restriction to our approach is our incapability of computing the Mordell-Weil group and the Tate-Shafarevich group. In order to obtain at least some numerical evidence, we will usually trust in the equivariant rank conjecture and thus assume that the analytic rank equals the geometric rank $r$ and compute $r$ by computing approximations to the $L$-values. Even here, our approach is rather vague, since we do not make use of a criterion that would allow us to decide whether an $L$-value is actually 0 from the knowledge of numerical approximations.

However, numerical computations can prove that the analytic rank is 0 , and in this case (assuming that $K$ is totally real), we can use results proved independently by 
Longo and by Tian and Zhang (see [Darmon 06, Theorem 3.7]) to deduce that the algebraic rank is also 0.

If $r=0$ or in the rare case that $r>0$ and we know generators for $E(K)$, we use the usual Birch and SwinnertonDyer conjecture to compute a conjectural value for \#Ш $(E / K)$. In a sense, our results can be characterized as "deducing numerical evidence for ETNC from the classical BSD-conjecture for $E / K$."

\section{COMPUTATIONAL REMARKS}

In this section we describe how we perform our computations.

\subsection{Computation of $L$-Values}

For the computation of the leading coefficients $L^{*}(E / \mathbb{Q}, \chi, 1), \quad \chi \in \operatorname{Irr}(G)$, we use the algorithm described in [Dokchitser 04]. Actually, we apply the MAGMA implementation of this algorithm.

We use the algorithm to compute complex approximations to the derivatives $L^{(k)}(E / \mathbb{Q}, \chi, 1)$ of the twisted Hasse-Weil $L$-functions at $s=1$. We also use these values to guess the order of vanishing of $L(E / \mathbb{Q}, \chi, s)$ at $s=1$ in a very naive way. Explicitly, we set

$$
\tilde{r}(\chi):=\min \left\{k \geq 0|| L^{(k)}(E / \mathbb{Q}, \chi, 1) \mid>\varepsilon\right\},
$$

where $\varepsilon>0$ is a chosen lower bound that seems to be reasonable in an unspecific way. At least one can hope that $\tilde{r}(\chi)$ is equal to the order of $L(E / \mathbb{Q}, \chi, s)$ at $s=1$.

\subsection{Computation of Periods}

For the computation of periods associated with $h^{1}\left(E_{K}\right)(1)$ considered as a motive over $\mathbb{Q}$ with coefficients in $\mathbb{Q}[G]$, we apply Proposition 3.1. The computation of $\Omega_{+}$and $\Omega_{-}$is standard, and we just use the implementation provided by MAGMA. It is usually very efficient to compute a normal basis element $\alpha_{0}$ just by trial and error. Without loss of generality, we assume that $\alpha_{0} \in \mathcal{O}_{K}$. Then the exceptional set $\mathrm{HP}_{2}$ contains

$$
\mathrm{HP}_{2}^{\prime}=\left\{l \mid l \text { divides }\left[\mathcal{O}_{K}: \mathbb{Z}[G] \alpha_{0}\right]\right\},
$$

which can be computed easily.

If we want to check the conjecture for primes $l \in \mathrm{HP}$, we must assume Hypotheses 4.1, in particular, that $l$ is at most tamely ramified in $K / \mathbb{Q}$. In this case, we can use [Bley and Wilson 09, Algorithm 4.2] to compute $\alpha_{0} \in$ $\mathcal{O}_{K}$ such that $\mathcal{O}_{K, l}=\mathbb{Z}_{l}[G] \alpha_{0}$.

However, it is reasonable to compute $u$ such that the exceptional set $\mathrm{HP}$ is as small as possible. Under certain assumptions on the group $G$ (which are satisfied, for example, for all groups with $\# G<32$ ), we can often use the methods of [Bley and Johnston 08, Bley and Johnston 11] to compute $\alpha_{0} \in \mathcal{O}_{K}$ such that $\mathrm{HP}_{2}^{\prime} \subseteq \mathrm{HP}_{1}$. This is possible because for small groups $G$, the ring of integers $\mathcal{O}_{K}$ is often free over the associated order

$$
\mathcal{A}=\mathcal{A}\left(\mathbb{Q}[G] ; \mathcal{O}_{K}\right):=\left\{\lambda \in \mathbb{Q}[G] \mid \lambda\left(\mathcal{O}_{K}\right) \subseteq \mathcal{O}_{K}\right\},
$$

and in this case, [Bley and Wilson 09, Algorithm 4.2] computes a free generator $\alpha_{0}$ such that $\mathcal{O}_{K}=\mathcal{A} \alpha_{0}$. Basic properties of associated orders then imply that $\mathcal{O}_{K, l}=$ $\mathbb{Z}_{l}[G] \alpha_{0}$ for all $l \nmid \# G$.

\subsection{Computation of Equivariant Regulators}

Our possibilities to compute regulators are very limited, because in most cases, we are not able to compute the Mordell-Weil group $E(K)$ when $K \neq \mathbb{Q}$ (or a subgroup of finite index in $E(K))$. Henceforth, we assume $r>0$ and that

$$
E(K)=E(K)_{\text {tors }} \oplus \mathbb{Z} P_{1} \oplus \cdots \oplus \mathbb{Z} P_{r}
$$

is explicitly known. Note, however, that for the rationality conjecture it would be enough to know a subgroup of finite index.

We consider $E(K) \otimes_{\mathbb{Z}} \mathbb{Q}$ and remind the reader of Remark 2.7(b), where the general recipe for the computation of regulators is described. However, for our actual computations described in the next section, it will be enough to consider irreducible rational characters $\chi \in \operatorname{Irr}_{\mathbb{Q}}(G)$ that factor through the commutator subgroup $G^{\prime}$. For all other characters, we assume that $e_{\chi}\left(E(K) \otimes_{\mathbb{Z}} \mathbb{Q}\right)$ is trivial. Under these circumstances, it is rather straightforward to compute a $\mathbb{Q}[G]$-basis in the sense of Remark 2.7(b). We describe the computation of the equivariant regulator in this case.

Let $\psi \in \operatorname{Irr}(G)$ denote an absolutely irreducible abelian character and set $F:=K^{\operatorname{ker}(\psi)}$. Let

$$
\chi=\sum_{\sigma \in \operatorname{Gal}(\mathbb{Q}(\psi) / \mathbb{Q})} \psi^{\sigma}
$$

be the associated rational character. Then $e_{\chi} \mathbb{Q}[G] \simeq$ $\mathbb{Q}(\psi)$ is a field and $e_{\chi}\left(E(K) \otimes_{\mathbb{Z}} \mathbb{Q}\right)=e_{\chi}\left(E(F) \otimes_{\mathbb{Z}} \mathbb{Q}\right)$ is a finite-dimensional $e_{\chi} \mathbb{Q}[G]$-vector space. Let $Q_{1}, \ldots, Q_{d}$ be a $e_{\chi} \mathbb{Q}[G]$-basis.

For an abelian character $\varphi$ we write $\varphi \mid \chi$ if $\varphi$ is a constituent of $\chi$. If

$$
a=\left(a_{\varphi}\right)_{\varphi \in \operatorname{Irr}(G)} \in \prod_{\varphi \in \operatorname{Irr}(G)} \simeq \zeta(\mathbb{C}[G]),
$$


then we write $a_{\chi}$ for the $\chi$-part

$$
\left(a_{\varphi}\right)_{\varphi \mid \chi} \in \prod_{\varphi \mid \chi} \simeq \zeta\left(e_{\chi} \mathbb{C}[G]\right) .
$$

Proposition 5.1. Assume the above notation. With respect to the $e_{\chi} \mathbb{Q}[G]$-bases $Q_{1}, \ldots, Q_{d}$ and $Q_{1}^{*}, \ldots, Q_{d}^{*}$ with $Q_{i}^{*}$ defined below in (5-1), the $\chi$-part of the regulator is given by

$$
\operatorname{Reg}_{\chi}:=\left(\operatorname{det}\left(\left\langle Q_{i}, e_{\bar{\psi}^{\sigma}} Q_{j}\right\rangle\right)_{1 \leq i, j \leq d}\right)_{\sigma \in \operatorname{Gal}(\mathbb{Q}(\psi) / \mathbb{Q})} .
$$

Proof. The set $\left\{e_{\varphi} Q_{i}|i=1, \ldots, d, \varphi| \chi\right\}$ is a $\mathbb{C}$-basis of $e_{\chi}\left(E(K) \otimes_{\mathbb{Z}} \mathbb{C}\right)$. We define the dual basis by

$$
\left(e_{\varphi} Q_{i}\right)^{*}\left(e_{\lambda} Q_{j}\right)= \begin{cases}1, & \text { if } \varphi=\lambda \text { and } i=j \\ 0, & \text { otherwise }\end{cases}
$$

Then one easily verifies that

$$
e_{\lambda}\left(e_{\varphi} Q_{i}\right)^{*}= \begin{cases}\left(e_{\varphi} Q_{i}\right)^{*}, & \text { if } \varphi=\bar{\lambda} \\ 0, & \text { otherwise }\end{cases}
$$

The elements

$$
Q_{i}^{*}:=\sum_{\varphi \mid \chi}\left(e_{\bar{\varphi}} Q_{i}\right)^{*}, \quad i=1, \ldots, d,
$$

form an $e_{\chi} \mathbb{Q}[G]$-basis of $\left(e_{\chi}\left(E(K) \otimes_{\mathbb{Z}} \mathbb{Q}\right)\right)^{*}$. Then

$$
\begin{aligned}
\left\langle e_{\varphi} Q_{i},{ }_{-}\right\rangle & =\sum_{j, \lambda}\left\langle e_{\varphi} Q_{i}, e_{\lambda} Q_{j}\right\rangle\left(e_{\lambda} Q_{j}\right)^{*} \\
& =\sum_{j, \lambda}\left\langle Q_{i}, e_{\bar{\varphi}} e_{\lambda} Q_{j}\right\rangle\left(e_{\lambda} Q_{j}\right)^{*} \\
& =\sum_{j}\left\langle Q_{i}, e_{\bar{\varphi}} Q_{j}\right\rangle\left(e_{\bar{\varphi}} Q_{j}\right)^{*} .
\end{aligned}
$$

Hence

$$
\begin{aligned}
\left\langle e_{\chi} Q_{i},{ }_{-}\right\rangle & =\sum_{\varphi \mid \chi} \sum_{j}\left\langle Q_{i}, e_{\bar{\varphi}} Q_{j}\right\rangle\left(e_{\bar{\varphi}} Q_{j}\right)^{*} \\
& =\sum_{j}\left(\sum_{\varphi \mid \chi}\left\langle Q_{i}, e_{\bar{\varphi}} Q_{j}\right\rangle e_{\varphi}\right)\left(\sum_{\varphi \mid \chi}\left(e_{\bar{\varphi}} Q_{j}\right)^{*}\right) \\
& =\sum_{j}\left(\sum_{\varphi \mid \chi}\left\langle Q_{i}, e_{\bar{\varphi}} Q_{j}\right\rangle e_{\varphi}\right) Q_{j}^{*} .
\end{aligned}
$$

Therefore, with respect to the bases $Q_{1}, \ldots, Q_{d}$ and $Q_{1}^{*}, \ldots, Q_{d}^{*}$, the regulator map is represented by the ma$\operatorname{trix}\left(\sum_{\varphi \mid \chi}\left\langle Q_{i}, e_{\bar{\varphi}} Q_{j}\right\rangle e_{\varphi}\right)_{1 \leq i, j \leq d}$, and the result follows upon computing the reduced norm.

For integrality considerations we restrict ourselves to the case that $l \neq 2$ and $l \nmid \# G$. Then $E(K) \otimes_{\mathbb{Z}} \mathbb{Z}_{l}$ is $\mathbb{Z}_{l}[G]$-perfect, and we wish to compute an $e_{\chi} \mathbb{Z}_{l}[G]$-basis $Q_{1}, \ldots, Q_{d}$ of $e_{\chi}\left(E(K) \otimes_{\mathbb{Z}} \mathbb{Z}_{l}\right)$. Since $e_{\chi} \mathbb{Z}_{l}[G]$ naturally identifies with $\prod_{\mathfrak{P} \mid l} \mathbb{Z}_{l}[\psi]$, which is a product of discrete valuation rings, such a basis always exists. From

$$
Q_{i}^{*}\left(Q_{j}\right)= \begin{cases}\chi(1), & \text { if } i=j \\ 0, & \text { if } i \neq j\end{cases}
$$

we see that

$$
\frac{1}{\chi(1)} Q_{1}^{*}, \quad \ldots, \quad \frac{1}{\chi(1)} Q_{d}^{*}
$$

is an $e_{\chi} \mathbb{Z}_{l}[G]$-basis of $e_{\chi}\left(E(K) \otimes_{\mathbb{Z}} \mathbb{Z}_{l}\right)^{*}$.

Working with localizations rather than completions, we can also consider $e_{\chi}\left(E(K) \otimes_{\mathbb{Z}} \mathbb{Z}_{(l)}\right)$ as a module over $e_{\chi} \mathbb{Z}_{(l)}[G] \simeq \mathbb{Z}_{(l)}[\psi] \subseteq \mathbb{Q}(\psi)$, which is a principal ideal ring (because it is Dedekind with only finitely many maximal ideals). It is then quite standard to compute a basis from the knowledge of $P_{1}, \ldots, P_{r}$.

Example 5.2. In [Fearnley and Kisilevsky 10], the authors consider the situation that $K / \mathbb{Q}$ is a cyclic extension of odd prime degree $l$ and examine the case that $L(E / \mathbb{Q}, \psi, s)$ has simple zeros for all nontrivial characters $\psi \in \operatorname{Irr}(G)$. We write $\hat{G}$ for the group of linear characters of $G$ and fix a generator $\psi_{0}$ of $\hat{G}$. Let $\chi:=\sum_{\gamma \in \operatorname{Gal}\left(\mathbb{Q}\left(\psi_{0}\right) / \mathbb{Q}\right)} \psi_{0}^{\gamma}$ denote the associated irreducible rational character. The computations described in [Fearnley and Kisilevsky 10] suggest that for nontrivial $\psi$ and a point $P \in E(K)$ of infinite order with trace 0 (i.e., $P \in e_{\chi}\left(E(K) \otimes_{\mathbb{Z}} \mathbb{Q}\right)$ is a $e_{\chi} \mathbb{Q}[G]$-basis), one has

$$
L^{\prime}(E / \mathbb{Q}, \psi, 1)=\frac{\tau(\psi)}{\mathfrak{f}_{\psi}} \Omega_{+} \lambda_{\psi}(P) \alpha_{\psi}(P),
$$

with a Gauss sum $\tau(\psi)$, the conductor $\mathfrak{f}_{\psi}$ of $\psi$,

$$
\lambda_{\psi}(P):=\sum_{\sigma \in G} \psi\left(\sigma^{-1}\right)\left\langle P, P^{\sigma}\right\rangle,
$$

and an algebraic number $\alpha_{\psi}(P) \in \mathbb{Q}(\psi)$ that satisfies $\alpha_{\psi \gamma}(P)=\alpha_{\psi}(P)^{\gamma}$ for all $\gamma \in \operatorname{Gal}(\mathbb{Q}(\psi) / \mathbb{Q})$. In other words, this means that $\left(\alpha_{\psi}(P)\right)_{\psi \mid \chi} \in \zeta\left(e_{\chi} \mathbb{Q}[G]\right)^{\times}$.

The results and computations of [Fearnley and Kisilevsky 10] are completely consistent with the rationality conjecture (Conjecture 3.5) and provide numerical evidence for it. Indeed, one easily shows that $\lambda_{\psi}(P)=\left\langle P, e_{\bar{\psi}} P\right\rangle$, so that we deduce from Proposition 5.1 that

$$
\operatorname{Reg}_{\chi} \equiv\left(\lambda_{\psi_{0}^{\gamma}}(P)\right)_{\gamma \in \operatorname{Gal}\left(\mathbb{Q}\left(\psi_{0}\right) / \mathbb{Q}\right)}
$$

where $\equiv$ means up to a multiplicative factor in $e_{\chi} \mathbb{Q}[G]^{\times} \subseteq \prod_{\psi \neq 1} \mathbb{C}^{\times}$. Furthermore, by [Fröhlich 89 , 
Section 9(i), (ii)] one has

$$
\begin{aligned}
& \left(\tau\left(\psi_{0}^{\gamma}\right)\right)_{\gamma \in \operatorname{Gal}\left(\mathbb{Q}\left(\psi_{0}\right) / \mathbb{Q}\right)} \\
& \quad \equiv\left(\sum_{\sigma \in G} \psi_{0}^{\gamma}(\sigma) \sigma\left(\alpha_{0}\right)\right)_{\gamma \in \operatorname{Gal}\left(\mathbb{Q}\left(\psi_{0}\right) / \mathbb{Q}\right)} .
\end{aligned}
$$

Recall that the right-hand side is exactly the $\chi$-part of the resolvent $R$ from Conjecture 3.5.

\subsection{Computation of Refined Euler Characteristics of Finite Perfect Modules}

Let $T$ be a finite perfect $\mathbb{Z}_{l}[G]$-module. The recipe for the computation of $\chi_{\mathbb{Z}_{l}[G], Q_{l}}(T)$ is already given in Example 2.8. We give two applications.

Proposition 5.3. Let $F / \mathbb{Q}_{p}$ denote a finite Galois extension with group $D$. Let $v$ denote the normalized discrete valuation of $F$. Let $E / \mathbb{Q}_{p}$ be an elliptic curve such that $E / F$ has split multiplicative reduction. Then

(a) $\# E(F) / E_{0}(F)=c$ with $c:=-v(j(E))$.

(b) Let l be a prime. Then $E(F) / E_{0}(F) \otimes_{\mathbb{Z}} \mathbb{Z}_{l}$ is $\mathbb{Z}_{l}[D]$ perfect if and only if $l \nmid c$ or $l \nmid \# D$.

(c) Set $c_{l}:=\#\left(E(F) / E_{0}(F) \otimes_{\mathbb{Z}} \mathbb{Z}_{l}\right)$. If $l \nmid c$ or $l \nmid \# D$, then $\chi_{\mathbb{Z}_{l}[D], \mathbb{Q}_{l}}\left(E(F) / E_{0}(F) \otimes_{\mathbb{Z}} \mathbb{Z}_{l}\right)$ is represented by $\left(c_{l}, 1, \ldots, 1\right) \in \zeta(\mathbb{Q}[D])^{\times}$.

Proof. We apply [Silverman 86, Theorem 14.1]. We see that $E$ is isomorphic over $F$ to the Tate curve $E_{q}$ with $v(q)=c$. The isomorphism $E_{q}(F) \simeq F^{\times} / q^{\mathbb{Z}}$ induces a $D$ equivariant isomorphism

$$
E(F) / E_{0}(F) \simeq F^{\times} /\left(q^{\mathbb{Z}} \times \mathcal{O}_{F}^{\times}\right) .
$$

Note that $D$ acts trivially on the right-hand side, so that $F^{\times} /\left(q^{\mathbb{Z}} \times \mathcal{O}_{F}^{\times}\right) \simeq \mathbb{Z} / c \mathbb{Z}$ as Galois modules. Parts (a) and (b) are now immediate. To prove (c), we may assume $l \nmid \# D$. Then we have a projective resolution of $\mathbb{Z}_{(l)}[D]-$ modules

$$
0 \longrightarrow \mathbb{Z}_{(l)}[D]^{c_{l} e_{D}+\left(1-e_{D}\right)} \mathbb{Z}_{(l)}[D] \longrightarrow \mathbb{Z} / c_{l} \mathbb{Z} \longrightarrow 0 .
$$

The result follows.

Proposition 5.4. Let $F / \mathbb{Q}_{l}$ denote a finite Galois extension with group $D$ and ramification subgroup $I$. Let $v$ denote the normalized discrete valuation of $F$. Let $k_{v}$ denote the residue class field. Then $k_{v}$ is $\mathbb{Z}_{l}[D]$-perfect if and only if $l \nmid \# I$. In this case, $\chi_{\mathbb{Z}_{l}[D], Q_{l}}\left(k_{v}\right)$ is represented by $\left(a_{\psi}\right)_{\psi} \in \zeta(\mathbb{Q}[D])^{\times} \simeq \prod_{\psi \in \operatorname{Irr}_{\mathbb{Q}}(D)} \mathbb{Q}(\psi)$ with

$$
a_{\psi}= \begin{cases}l, & \text { if } I \subseteq \operatorname{ker}(\psi), \\ 1, & \text { otherwise }\end{cases}
$$

Proof. By the normal basis theorem, one has $k_{v} \simeq$ $\mathbb{F}_{l}[D / I]$. One easily shows that $\hat{H}^{0}\left(D, \mathbb{F}_{l}[D / I]\right) \simeq$ $\mathbb{F}_{l} /|I| \mathbb{F}_{l}$. It follows that if $k_{v}$ is perfect, then $l \nmid \# I$. Conversely, if $l \nmid \# I$, then we have the projective resolution

$$
0 \longrightarrow \mathbb{Z}_{l}[D] \stackrel{l e_{I}+\left(1-e_{I}\right)}{\longrightarrow} \mathbb{Z}_{l}[D] \longrightarrow k_{v} \longrightarrow 0,
$$

and the result follows.

In general, we assume that the finite perfect $\mathbb{Z}_{l}[G]$ module $T$ is given by a $\mathbb{Z}[G]$-generating set $t_{1}, \ldots, t_{d}$ with explicitly known $G$-action, i.e.,

$$
g t_{i}=\sum_{j=1}^{d} a_{g, j} t_{j}, \quad a_{g, j} \in \mathbb{Z}[G], g \in G .
$$

It is then easy to compute a $\mathbb{Z}[G]$-resolution of the form

$$
0 \longrightarrow Q \longrightarrow P \stackrel{\pi}{\longrightarrow} T \longrightarrow 0
$$

with $P:=\mathbb{Z}[G]^{d}, \pi\left(e_{i}\right):=t_{i}$, where $e_{i}$ denotes the canonical basis and $Q:=\operatorname{ker}(\pi)$. We then proceed as described in Example 2.8.

In this way, it is, in principle, possible to compute the refined Euler characteristics of $E(K)_{l^{\infty}}, E(K)_{l^{\infty}}^{\vee}$, and $\bar{E}_{\mathrm{ns}}\left(k_{v_{p}}\right)_{l^{\infty}}$, at least in small examples where we are able to provide an explicit generating set with explicitly known $G$-action. If $v$ is a place of bad reduction, we can use Tate's algorithm to determine the reduction type and then use [Silverman 86, Exercise III, 3.5] in order to compute $\bar{E}_{\mathrm{ns}}\left(k_{v}\right)_{l^{\infty}}$.

\subsection{Computation of $E(K)$ and $\amalg(E / K)$}

The computation of $E(K)$ is very difficult even if $K=\mathbb{Q}$, and it usually does not work if $K \neq \mathbb{Q}$. In our examples, we mostly consider pairs $(E, K)$ such that the analytic rank of $E / K$ is 0 and $K$ is totally real. In this case, we use [Darmon 06, Theorem 3.7] to deduce that the algebraic rank is also equal to 0 . In small examples (see, e.g., Section 6.1), it is sometimes possible to prove that the algebraic rank is trivial by a Selmer group computation.

If $r>0$, we generally assume the validity of the equivariant rank conjecture and consider only examples in which the equivariant rank conjecture implies that $E(K)$ is built from subgroups $E(F)$ where $F$ ranges over the subfields of $K / \mathbb{Q}$ with $[F: \mathbb{Q}] \leq 2$. For $F=\mathbb{Q}$, we use the Magma routine to compute $E(\mathbb{Q})$, and if $F$ is a quadratic extension, we look at the associated quadratic twist $E_{d}$ of $E$ and compute $E_{d}(\mathbb{Q})$. Computing the isomorphism $E \simeq E_{d}$ (defined over $F$ ), we then obtain $E(F)$.

Our ability to compute $\amalg(E / K)$ is even more limited. We remind the reader that throughout the manuscript we assume finiteness of $\amalg(E / K)$. In order to compute 
$\# \amalg(E / K)$, we use the classical BSD conjecture for $E / K$. In this way, we obtain a conjectural value for $\# \amalg(E / K)$ that we wish to use to compute the associated refined Euler characteristic. Since we have at our disposal only the (conjectural) order of $\amalg(E / K)$, we are usually restricted to dealing with primes $l$ that do not divide this order. However, in some rare cases (see, e.g., Section 6.2), it suffices to know this order to compute the refined Euler characteristic of $\amalg(E / K)$. Moreover, in some examples (see Sections 6.1 and 6.3), the computations lead to a conjectural description of the structure of $\amalg(E / K)$ as a Galois module.

\subsection{Computation of Induction}

If $H$ is a subgroup of $G$, then there is a canonical induction $\operatorname{map} \operatorname{ind}_{H}^{G}: K_{0}\left(\mathbb{Z}_{l}[H], \mathbb{Q}_{l}\right) \longrightarrow K_{0}\left(\mathbb{Z}_{l}[G], \mathbb{Q}_{l}\right)$. We refer the reader to [Bley and Wilson 09, Section 6], where we provide an algorithmic description of this map.

\section{EXAMPLES}

In this section we illustrate our results with some explicit examples. The computational results of this section can be reproduced using the author's MAgma implementations. $^{2}$

\subsection{Navilarekallu's Example}

In this subsection we redo the example from [Navilarekallu 88]. Let

$$
E: y^{2}+y=x^{3}-x^{2}-10 x-20,
$$

and let $K$ be the splitting field of $f(x)=x^{3}-4 x+1$. Then $K / \mathbb{Q}$ is an $S_{3}$-extension. The elliptic curve $E$ is denoted by $11 A 1$ in Cremona's database. Its conductor is $N_{E}=11$, and the discriminant of $K$ is given by $d_{K / \mathbb{Q}}=229^{3}$. The field $K$ is totally real and contains the quadratic subfield $F:=\mathbb{Q}(\sqrt{229})$. In fact, $K$ is the Hilbert class field of $F$.

We have $S=\{11,229\}$. For a rational prime $q$, we fix a place $v_{q}$ of $K$ above $q$. One easily computes

$$
\begin{aligned}
\# \bar{I}_{229} & =2, \quad \# \bar{E}_{\mathrm{ns}}\left(k_{v_{2}}\right)=5, \\
\# \bar{E}_{\mathrm{ns}}\left(k_{v_{3}}\right) & =20, \quad \# \bar{E}_{\mathrm{ns}}\left(k_{v_{5}}\right)=140, \\
\# \bar{E}_{\mathrm{ns}}\left(k_{v_{11}}\right) & =1330, \quad \# \bar{E}_{\mathrm{ns}}\left(k_{v_{22}}\right)=215, \\
E(K)_{\text {tors }} & =E(\mathbb{Q})_{\text {tors }} \text { is cyclic of order } 5,
\end{aligned}
$$

\footnotetext{
${ }^{2}$ Available online at http://www.mathematik.uni-muenchen.de/ $\sim$ bley/pub.html
}

$E$ has split multiplicative reduction at $v_{11}$ with $c_{v_{11}}=5$,

$$
\begin{aligned}
\left(L_{11}(E / Q, \bar{\chi}, 1)\right) & =(10 / 11,10 / 11,133 / 121), \\
\left(L_{229}(E / Q, \bar{\chi}, 1)\right) & =(215 / 229,1,215 / 229),
\end{aligned}
$$

and

$$
\begin{aligned}
\operatorname{ind}_{G_{v_{11}}}^{G} \chi_{G_{v_{11}}}\left(k_{v_{11}}\right) & =(11,11,121), \\
\operatorname{ind}_{G_{v_{22}}}^{G} \chi_{G_{v_{11}}}\left(k_{v_{229}}\right) & =(229,1,229) .
\end{aligned}
$$

The $L$-values can easily be computed with a precision of 20 or more decimal digits. We give here only the first six decimal digits: $(L(E / \mathbb{Q}, \bar{\chi}, 1))_{\chi \in \operatorname{Irr}_{Q}(G)}=$ $(0.253842,0.419359,2.66127)$. Therefore, the analytic rank for each of the $L$-functions is trivial. By [Darmon 06, Theorem 3.7] (which has been proved independently by Longo and by Tian and Zhang), we have $E(K)=$ $E(K)_{\text {tors }}=E(\mathbb{Q})_{\text {tors }}$.

In this small case, this can also be proved by algorithmic methods. Let $K_{1}$ be the number field defined by $f(x)=x^{3}-4 x+1$. Then the computation of Selmer groups using the MAGMA routine TwoSelmerGroup shows that $\mathrm{Sel}^{(2)}\left(E / K_{1}\right)$ and $\mathrm{Sel}^{(2)}(E / F)$ are trivial. It follows quite easily that $E(K)$ must be torsion.

We obtain

$$
u=(0.200000,-5.00000,-25.0000),
$$

which numerically confirms the rationality conjecture. We point out that the resolvents and therefore also the value for $u$ depend on the choice of the integral normal basis element $\alpha_{0}$. The algorithm of [Bley and Johnston 08] does not always produce the same generator, so that one may obtain different results when running the algorithm. Note, however, that the validity of the ETNC does not depend on this choice (see Remark 4.7).

From the BSD conjecture we conclude the conjectural order $\# \amalg(E / K)=625$, so that $\mathrm{HP}=\{2,3,5,11,229\}$. By Corollary 4.8 we immediately obtain a numerical confirmation for all primes $l \notin \mathrm{HP}$.

For $l=2$, Hypothesis 4.1(2a) is not satisfied, and for $l=5$, we do not have Hypothesis 4.1(5). For $l=3$, we have $\xi_{l}=(1,1,1)$. So $u=u \xi_{l}^{-1}$ is a torsion element in $K_{0}\left(\mathbb{Z}_{3}[G], \mathbb{Q}_{3}\right)$. Here $K_{0}\left(\mathbb{Z}_{3}[G], \mathbb{Q}_{3}\right)_{\text {tors }}$ is cyclic of order 2, and by the methods of [Bley and Wilson 09], we can check that $u$ is indeed trivial in this group. We can also directly check the explicit congruence (2-6), which becomes $-1 \equiv-25(\bmod 3)$ in this example.

For $l \in\{11,229\}$ the group $K_{0}\left(\mathbb{Z}_{l}[G], \mathbb{Q}_{l}\right)_{\text {tors }}$ is trivial, and from the above data, one easily deduces the validity of the $l$-part of ETNC. 
Although we do not have Hypothesis 4.1(5) for $l=5$, one can try to proceed as in Remark 4.4. By Proposition 5.3, we can compute the refined Euler characteristic of $\mathcal{C}$ (see Remark 4.4 for the notation). Since we do not know how to compute the Euler characteristics of $\mathcal{S}$ and $H_{f}^{1}\left(\mathbb{Q}, T_{l}\right)^{*}$ rigorously, we get only a very vague idea about the Galois structure of $\amalg(E / K)$. However, by the conjectural validity of ETNC at $l=5$, one is tempted to guess that $\tilde{\chi}\left(\amalg(E / K)_{5^{\infty}}\right)=\left(1,1, \frac{1}{25}\right)$. One may therefore guess that $\amalg(E / K)_{5}$ lives in the 2-dimensional component of $\mathbb{Q}_{5}[G]$ and has the resolution

$$
0 \longrightarrow M_{2}\left(\mathbb{Z}_{5}\right) \stackrel{\left(\begin{array}{ll}
5 & 0 \\
0 & 5
\end{array}\right)}{\longrightarrow} M_{2}\left(\mathbb{Z}_{5}\right) \longrightarrow \amalg(E / K)_{5^{\infty}} \longrightarrow 0 .
$$

This was verified by T. Fisher in a private communication.

\subsection{A $D_{5}$ Example}

In this example we let $E$ be the curve $73 A 1$ in Cremona's notation. We let $K$ be the number field defined by the irreducible polynomial

$$
\begin{aligned}
f(x)= & x^{10}-2 x^{9}-20 x^{8}+2 x^{7}+69 x^{6}-x^{5}-69 x^{4}+2 x^{3} \\
& +20 x^{2}-2 x-1 .
\end{aligned}
$$

Then $K / \mathbb{Q}$ is a Galois extension with dihedral group $D_{5}$. We have $N_{E}=73$ and $d_{K / \mathbb{Q}}=401^{5}$. The field $K$ is totally real and contains the quadratic subfield $F:=$ $\mathbb{Q}(\sqrt{401})$. Hence $S=\{73,401\}$. In fact, $K$ is the Hilbert class field of $F$.

We have four characters:

\begin{tabular}{c|cccc} 
& id & $\tau$ & $\sigma$ & $\sigma^{2}$ \\
\hline$\chi_{1}$ & 1 & 1 & 1 & 1 \\
$\chi_{2}$ & 1 & -1 & 1 & 1 \\
$\chi_{3}$ & 2 & 0 & $\zeta_{5}+\zeta_{5}^{-1}$ & $\zeta_{5}^{2}+\zeta_{5}^{-2}$ \\
$\chi_{4}$ & 2 & 0 & $\zeta_{5}^{2}+\zeta_{5}^{-2}$ & $\zeta_{5}+\zeta_{5}^{-1}$
\end{tabular}

Hence $\mathbb{Q}[G] \simeq \mathbb{Q} \oplus \mathbb{Q} \oplus M_{2}\left(K_{3}\right)$ with $K_{3}=\mathbb{Q}(\beta)$, where $\beta:=\zeta_{5}+\zeta_{5}^{-1}$. Elements in the center of $\mathbb{C}[G]$ will be denoted by 4 -tuples $z=\left(z_{1}, \ldots, z_{4}\right), z_{i} \in \mathbb{C}$. Recall that $z \in \zeta(\mathbb{Q}[G])$ if and only if $z_{1}, z_{2} \in \mathbb{Q}, z_{3}, z_{4} \in K_{3}$, and $\varphi\left(z_{3}\right)=z_{4}$, where $\langle\varphi\rangle=\operatorname{Gal}\left(K_{3} / \mathbb{Q}\right)$. Elements in $z \in$ $\zeta(\mathbb{Q}[G])$ will be represented by tuples $z=\left(z_{\chi_{1}}, z_{\chi_{2}}, z_{\chi_{3}}\right)$.

The $L$-values were computed with a precision of 20 decimal digits and are given by

$$
\begin{aligned}
(L(E / \mathbb{Q}, \bar{\chi}, 1))_{\chi \in \operatorname{Irr}_{\mathbb{Q}}(G)} & \\
= & (1.1826604672413298661,2.1261328339601570537 \\
& 0.16304872052191552777,7.6598191709443800630)
\end{aligned}
$$

The analytic rank of each of the twisted $L$-functions is therefore 0, and as in the first example, [Darmon 06, Theorem 3.7] allows us to conclude that $E(K)$ is finite.

The numerical computation of $L$-values, resolvents, and periods leads to

$$
\begin{array}{r}
u=(0.49999999999999999999,18.000000000000000008, \\
0.58359213500126187216,27.416407864998738187) .
\end{array}
$$

Numerically, this confirms the rationality conjecture, because $u$ is close to

$$
(1 / 2,18,-12 \beta+8,12 \beta+20),
$$

and $\varphi(-12 \beta+8)=12 \beta+20$. The minimal polynomial of $-12 \beta+8$ is given by $x^{2}-28 x+16$. Again we point out that the resolvents and therefore also the value for $u$ depend on the choice of the integral normal basis element $\alpha_{0}$.

We further see that

$$
\begin{aligned}
\# \bar{I}_{401} & =2, \quad \# \bar{E}_{\mathrm{ns}}\left(k_{v_{2}}\right)=22, \\
\# \bar{E}_{\mathrm{ns}}\left(k_{v_{3}}\right) & =16, \quad \# \bar{E}_{\mathrm{ns}}\left(k_{v_{5}}\right)=3044 \\
\# \bar{E}_{\mathrm{ns}}\left(k_{v_{73}}\right) & =2073071592, \quad \# \bar{E}_{\mathrm{ns}}\left(k_{v_{401}}\right)=388, \\
E(K)_{\text {tors }} & =E(\mathbb{Q})_{\text {tors }} \text { is cyclic of order } 2,
\end{aligned}
$$

$E$ has split multiplicative reduction at $v_{73}$ with $c_{v_{73}}=2$,

$$
\begin{aligned}
&\left(L_{73}(E / Q, \bar{\chi}, 1)\right) \\
&=\left(\frac{72}{73}, \frac{72}{73}, \frac{1}{5329}(73 \beta+5403), \frac{1}{5329}(-73 \beta+5330)\right), \\
&\left(L_{401}(E / Q, \bar{\chi}, 1)\right)=\left(\frac{388}{401}, 1, \frac{388}{401}, \frac{388}{401}\right), \\
& \operatorname{ind}_{G_{v_{73}}}^{G} \chi_{G_{v_{11}}}\left(k_{v_{11}}\right)=(73,73,5329),
\end{aligned}
$$

and

$$
\operatorname{ind}_{G_{v_{401}}}^{G} \chi_{G_{v_{401}}}\left(k_{v_{401}}\right)=(401,1,401) .
$$

Recall that any element $z \in \zeta(\mathbb{Q}[G])$ is represented by a tuple $z=\left(z_{\chi_{1}}, z_{\chi_{2}}, z_{\chi_{3}}\right)$. This explains why some of the above tuples have only three components.

From the BSD conjecture we derive the conjectural order $\# \amalg(E / K)=2304=2^{8} 3^{2}$. Thus we have $\mathrm{HP}=$ $\{2,3,5,73,401\}$, and by Corollary 4.8 , the ETNC is numerically confirmed outside HP.

For $l=2$ we cannot perform our computations, because 2 divides $\# G$.

For $l=3$, our MAGma implementation terminates without verifying the 3 -part of ETNC, because 3 divides the order of $\amalg(E / K)$. In general, we are not able to compute the refined Euler characteristic of $\amalg(E / K)$ if $l$ divides $\# \amalg(E / K)$, because we have no information about its Galois structure. However, in some special 
cases like this one, it is possible to pin down the exact Euler characteristic by purely representation-theoretic considerations. Here $\amalg(E / K)[3]$ is conjecturally bicyclic of order 9, and we may consider it a representation over $\mathbb{F}_{3}$. There are three irreducible representations over $\mathbb{F}_{3}$, namely the trivial character, the sign character, and a 2-dimensional representation (defined over $\left.\mathbb{F}_{9}\right)$. A BSD-computation for $E / \mathbb{Q}$ and $E / F$ shows that $\# \amalg(E / \mathbb{Q})[3]=1$ and $\# \amalg(E / F)=9$, so that we obtain $\tilde{\chi}(\amalg(E / K))=(1,9,1)$. Using this, we can also confirm the validity of ETNC at $l=3$.

For $l \in\{73,401\}$, we have that $K_{0}\left(\mathbb{Z}_{l}[G], \mathbb{Q}_{l}\right)_{\text {tors }}$ is trivial, and from the above data one easily deduces the validity of the $l$-part of the ETNC.

Most interesting is the case $l=5$, because in this case we have the nontrivial torsion subgroup $K_{0}\left(\mathbb{Z}_{l}[G], \mathbb{Q}_{l}\right)_{\text {tors }}$, so that we must verify the explicit congruence $(2-6)$. We have $\xi_{5}=(1,1,1)$, so that $\eta:=u \xi_{5}^{-1}=$ $(1 / 2,18,-12 \beta+8)$. Let $\mathfrak{p}$ denote the unique prime lying over 5 in $K_{3}$. Then one easily checks that the valuation at $\mathfrak{p}$ of $\frac{1}{2} \cdot 18-(-12 \beta+8)$ equals 1 , as predicted by the ETNC.

\subsection{A $D_{7}$ Example}

In this example we let $E$ be the curve $11 A 1$ in Cremona's notation. We let $K$ be the number field defined by the irreducible polynomial

$$
\begin{aligned}
f(x)= & x^{14}-2 x^{13}-25 x^{12}+69 x^{11}+161 x^{10} 632 x^{9} \\
& -147 x^{8}+2146 x^{7}-1171 x^{6}-2669 x^{5}+2682 x^{4} \\
& +667 x^{3}-1466 x^{2}+336 x+49 .
\end{aligned}
$$

Then $K / \mathbb{Q}$ is a Galois extension with dihedral group $D_{7}$. We have $N_{E}=11$ and $d_{K / \mathbb{Q}}=577^{7}$. The field $K$ is totally real and contains the quadratic subfield $F:=$ $\mathbb{Q}(\sqrt{577})$. Hence $S=\{11,577\}$. In fact, $K$ is the Hilbert class field of $F$.

We have five characters:

\begin{tabular}{c|ccccc} 
& id & $\tau$ & $\sigma$ & $\sigma^{2}$ & $\sigma^{3}$ \\
\hline$\chi_{1}$ & 1 & 1 & 1 & 1 & 1 \\
$\chi_{2}$ & 1 & -1 & 1 & 1 & 1 \\
$\chi_{3}$ & 2 & 0 & $\zeta_{7}+\zeta_{7}^{-1}$ & $\zeta_{7}^{2}+\zeta_{7}^{-2}$ & $\zeta_{7}^{3}+\zeta_{7}^{-3}$ \\
$\chi_{4}$ & 2 & 0 & $\zeta_{7}^{2}+\zeta_{7}^{-2}$ & $\zeta_{7}^{4}+\zeta_{7}^{-4}$ & $\zeta_{7}^{6}+\zeta_{7}^{-6}$ \\
$\chi_{5}$ & 2 & 0 & $\zeta_{7}^{3}+\zeta_{7}^{-3}$ & $\zeta_{7}^{6}+\zeta_{7}^{-6}$ & $\zeta_{7}^{2}+\zeta_{7}^{-2}$
\end{tabular}

Hence $\mathbb{Q}[G] \simeq \mathbb{Q} \oplus \mathbb{Q} \oplus M_{2}\left(K_{3}\right)$ with $K_{3}=\mathbb{Q}(\beta)$, where $\beta:=\zeta_{7}+\zeta_{7}^{-1}$. Elements in the center of $\mathbb{C}[G]$ will be denoted by 5 -tuples $z=\left(z_{1}, \ldots, z_{5}\right), z_{i} \in \mathbb{C}$. Recall that $z \in \zeta(\mathbb{Q}[G])$ if and only if $z_{1}, z_{2} \in \mathbb{Q}, z_{3}, z_{4}, z_{5} \in K_{3}$ are
Galois conjugates. Elements in $z \in \zeta(\mathbb{Q}[G])$ will be represented by tuples $z=\left(z_{\chi_{1}}, z_{\chi_{2}}, z_{\chi_{3}}\right)$.

The $L$-values were computed with a precision of 30 decimal digits and are given by

$$
\begin{aligned}
(L(E / \mathbb{Q}, \bar{\chi}, 1))_{\chi \in \operatorname{Irr}_{\mathbb{Q}}(G)} & (0.253841860855910684337758923351, \\
& 0.264189373454632540506329085616, \\
& 8.46480303158617169018788040257, \\
& 1.07820141250454111015938289065, \\
& 0.516343882321445768698269093336) .
\end{aligned}
$$

The analytic rank of each of the twisted $L$-functions is therefore 0, and as before, [Darmon 06, Theorem 3.7] allows us to conclude that $E(K)$ is finite.

The numerical computation of $L$-values, resolvents, and periods leads to

$$
\begin{aligned}
u=( & -0.199999999999999999999999958641, \\
& -5.00000000000000000000000004909, \\
& 126.222933488057632838305516431, \\
& 16.0776033026947639028170113251, \\
& 7.69946320924760325930251071912) .
\end{aligned}
$$

Numerically, this confirms the rationality conjecture, because $u$ is close to

$$
\left(-1 / 5,-5,25 \beta^{2}+50 \beta+25,-50 \beta^{2}-25 \beta+125,25 \beta^{2}-25 \beta\right)
$$

and the last three components are Galois conjugates. The minimal polynomial of $25 \beta^{2}+50 \beta+25$ is given by $x^{3}-$ $150 x^{2}+3125 x-15625$. Once again we point out that the resolvents and therefore also the value for $u$ depend on the choice of the integral normal basis element $\alpha_{0}$. We further see that

$$
\begin{aligned}
\# \bar{I}_{577} & =2, \quad \# \bar{E}_{\mathrm{ns}}\left(k_{v_{2}}\right)=145 \\
\# \bar{E}_{\mathrm{ns}}\left(k_{v_{5}}\right) & =35, \quad \# \bar{E}_{\mathrm{ns}}\left(k_{v_{7}}\right)=60 \\
\# \bar{E}_{\mathrm{ns}}\left(k_{v_{11}}\right) & =19487170, \# \bar{E}_{\mathrm{ns}}\left(k_{v_{577}}\right)=545 \\
E(K)_{\text {tors }} & =E(\mathbb{Q})_{\text {tors }} \text { is cyclic of order } 5,
\end{aligned}
$$

$E$ has split multiplicative reduction at $v_{73}$ with $c_{v_{73}}=5$,

$$
\begin{aligned}
\left(L_{11}(E / Q, \bar{\chi}, 1)\right) & =\left(\frac{10}{11}, \frac{10}{11}, \frac{1}{121}\left(-11 \beta^{2}+144\right)\right), \\
\left(L_{577}(E / Q, \bar{\chi}, 1)\right) & =\left(\frac{545}{577}, 1, \frac{545}{577}\right), \\
\operatorname{ind}_{G_{v_{11}}}^{G} \chi_{G_{v_{11}}}\left(k_{v_{11}}\right) & =(11,11,121),
\end{aligned}
$$

and

$$
\operatorname{ind}_{G_{v_{577}}}^{G} \chi_{G_{v_{577}}}\left(k_{v_{577}}\right)=(577,1,577) .
$$


Recall that any element $z \in \zeta(\mathbb{Q}[G])$ is represented by a tuple $z=\left(z_{\chi_{1}}, z_{\chi_{2}}, z_{\chi_{3}}\right)$. This explains why the above tuples have only three components.

From the BSD conjecture we derive the conjectural order $\# \amalg(E / K)=5^{12}$. Thus we have $\mathrm{HP}=$ $\{2,5,7,11,577\}$, and by Corollary 4.8 , the ETNC is numerically confirmed outside HP.

For $l=2$ we cannot perform our computations, because 2 divides \#G.

For $l=5$ we cannot perform the computations because 5 divides the order of $\amalg(E / K)$ and the Tamagawa numbers. As in Example 6.1, we try to proceed as in Remark 4.4. By the conjectural validity of ETNC at $l=5$ one may guess that $\tilde{\chi}\left(\amalg(E / K)_{5^{\infty}}\right)=\left(1,1, \frac{1}{25}\right)$. One may therefore guess that $\amalg(E / K)_{5^{\infty}}$ lives in the 2-dimensional component of $\mathbb{Q}_{5}[G]$ and has the resolution

$$
\begin{aligned}
0 \longrightarrow M_{2}\left(\mathbb{Z}_{5}\left[\zeta_{7}\right]^{+}\right) & \stackrel{\left(\begin{array}{ll}
5 & 0 \\
0 & 5
\end{array}\right)}{\longrightarrow} M_{2}\left(\mathbb{Z}_{5}\left[\zeta_{7}\right]^{+}\right) \\
& \longrightarrow \amalg(E / K)_{5^{\infty}} \longrightarrow 0 .
\end{aligned}
$$

Note that $\mathbb{Z}_{5}\left[\zeta_{7}\right]^{+} / 5 \mathbb{Z}_{5}\left[\zeta_{7}\right]^{+}=\mathbb{F}_{5^{3}}$, so that this matches with the conjectural order of $\amalg(E / K)$.

For $l \in\{11,577\}$, the subgroup $K_{0}\left(\mathbb{Z}_{l}[G], \mathbb{Q}_{l}\right)_{\text {tors }}$ is trivial, and from the above data one easily deduces the validity of the $l$-part of the ETNC.

The most interesting prime is $l=7$, because in this case we have nontrivial torsion subgroup $K_{0}\left(\mathbb{Z}_{l}[G], \mathbb{Q}_{l}\right)_{\text {tors }}$, so that we must verify the explicit congruence $(2-6)$. We have $\xi_{7}=(1,1,1)$, so that $\eta:=u \xi_{7}^{-1}=$ $\left(-1 / 5,-5,25 \beta^{2}+50 \beta+25\right)$. Let $\mathfrak{p}$ denote the unique prime lying over 7 in $K_{3}$. Then one easily checks that the valuation at $\mathfrak{p}$ of $\frac{-1}{5} \cdot(-5)-\left(25 \beta^{2}+50 \beta+25\right)$ equals 1 , as predicted by the ETNC.

\subsection{More Dihedral Examples}

We have numerically checked a few more $D_{l}$ examples that are completely analogous to the previous examples. We searched for cases in which we could apply our methods for the prime $l$. In particular, we needed the Mordell-Weil group $E(K)$ to be finite. In all our examples, $K$ is a totally real number field, so that we can apply [Darmon 06, Theorem 3.7]. In all the examples, our computations numerically confirm the $l$-part of ETNC.

In the following we list our examples. In each of our examples, $K$ is the Hilbert class field of the real quadratic field $\mathbb{Q}(\sqrt{d})$. The elliptic curve is referenced as in Cre- mona's tables:

\begin{tabular}{ccc} 
& $d$ & $E$ \\
\hline$D_{3}$ & 229 & $11 a 1$ \\
& 229 & $17 a 1$ \\
& 257 & $11 a 1$ \\
& 257 & $17 a 1$ \\
& 733 & $17 a 1$ \\
& 761 & $17 a 1$ \\
\hline$D_{5}$ & $19 \cdot 43$ & $17 a 1$ \\
& $19 \cdot 43$ & $37 b 1$ \\
& $7 \cdot 199$ & $17 a 1$ \\
$7 \cdot 199$ & $19 a 1$ \\
& 1429 & $17 a 1$ \\
& 1429 & $19 a 1$ \\
\hline$D_{7}$ & 577 & $11 a 1$ \\
& 577 & $17 b 1$ \\
& 577 & $19 a 1$ \\
& 1009 & $37 b 1$
\end{tabular}

With more effort it is certainly possible to compute more examples. We refer the interested reader to the author's batch files. ${ }^{3}$

\subsection{Another $D_{5}$ Example (Incomplete)}

In this example we again let $E$ be the curve $11 A 1$ in Cremona's notation. We take the same number field $K$ as in the first $D_{5}$ example, namely the Hilbert class field of $F:=\mathbb{Q}(\sqrt{401})$. We have $N_{E}=11$ and $d_{K / \mathbb{Q}}=401^{4}$. Hence $S=\{11,401\}$.

Recall that $\mathbb{Q}[G] \simeq \mathbb{Q} \oplus \mathbb{Q} \oplus M_{2}\left(K_{3}\right) \quad$ with $K_{3}=\mathbb{Q}(\beta)$, where $\beta:=\zeta_{5}+\zeta_{5}^{-1}$.

The computation of $L$-values shows that conjecturally,

$$
\operatorname{ord}_{s=1}\left(L\left(E / \mathbb{Q}, \chi_{i}, s\right)\right)= \begin{cases}0 & \text { for } i=1,3,4 \\ 2 & \text { for } i=2 .\end{cases}
$$

The leading terms in the Taylor expansion of the twisted $L$-series were computed with a precision of 20 decimal digits and are given by

$$
\begin{aligned}
\left(L^{*}(E / \mathbb{Q}, \bar{\chi}, 1)\right)_{\chi \in \operatorname{Irr}_{\mathbb{Q}}(G)} & (0.25384186085591068434,11.064607087619745148, \\
& 5.2651360430010329737,0.76817299610176707595) .
\end{aligned}
$$

The validity of the rank conjecture would imply that the $\chi_{2}$-eigenspace of $E(K) \otimes_{\mathbb{Z}} \mathbb{Q}$ is 2-dimensional.

\footnotetext{
${ }^{3}$ Available online at http://www.mathematik.uni-muenchen.de/ $\sim$ bley/pub.html
} 
Since $K^{\operatorname{ker}\left(\chi_{2}\right)}=\mathbb{Q}(\sqrt{401})=: F$, this implies that conjecturally, $\operatorname{rk}(E(F))=2$. By considering the quadratic twist of $E / \mathbb{Q}$,

$$
E_{401}: y^{2}=x^{3}-2153446992 x-69667552958832,
$$

we compute

$$
E(F) / E(F)_{\text {tors }}=\left\langle P_{1}, P_{2}\right\rangle
$$

with

$$
P_{1}=\left(\frac{74}{9}, \frac{53}{54} \sqrt{401}-\frac{1}{2}\right), \quad P_{2}=\left(6, \frac{1}{2} \sqrt{401}-\frac{1}{2}\right) .
$$

One checks that the conjugate of $P_{i}$ is $-P_{i}$ for $i=1,2$, so that by Proposition 5.1, we obtain for the $\chi_{2}$-part of the equivariant regulator,

$$
\operatorname{det}\left(\left(\left\langle P_{i}, P_{j}\right\rangle\right)_{i, j \in\{1,2\}}\right)=34.914427985010413291 .
$$

Possibly, $\left\langle P_{1}, P_{2}\right\rangle$ is not the full Mordell-Weil group $E(K)$. However, if we content ourselves with checking the rationality conjecture, then this information is enough.

Together with the computations of $L$-values, resolvents, and periods we obtain

$u=(0.20000000000000000000,-5.0000000000000000017$,

- 65.450849718747376977, -9.5491502812526296414).

Numerically, this confirms the rationality conjecture, because $u$ is close to

$$
(1 / 5,-5,-25 \beta-50,25 \beta-25)
$$

and $\varphi(-25 \beta-5)=25 \beta-25$. The minimal polynomial of $-25 \beta-5$ is given by $x^{2}+75 x+625$. Again we note that $u$ also depends on the choice of $\alpha_{0}$.

We further see that

$$
\begin{aligned}
\# \bar{I}_{401} & =2, \quad \# \bar{E}_{\mathrm{ns}}\left(k_{v_{2}}\right)=25, \quad \# \bar{E}_{\mathrm{ns}}\left(k_{v_{5}}\right)=3025, \\
\# \bar{E}_{\mathrm{ns}}\left(k_{v_{11}}\right) & =161050, \# \bar{E}_{\mathrm{ns}}\left(k_{v_{401}}\right)=400, \\
E(K)_{\text {tors }} & =E(\mathbb{Q})_{\text {tors }} \text { is cyclic of order } 5,
\end{aligned}
$$

$E$ has split multiplicative reduction at $v_{11}$ with $c_{v_{11}}=5$,

$$
\begin{aligned}
\left(L_{11}(E / Q, \bar{\chi}, 1)\right) & =\left(\frac{10}{11}, \frac{10}{11}, \frac{1}{121}(-11 \beta+122)\right), \\
\left(L_{401}(E / Q, \bar{\chi}, 1)\right) & =\left(\frac{400}{401}, 1, \frac{400}{401}\right), \\
\operatorname{ind}_{G_{v_{11}}}^{G} \chi_{G_{v_{11}}}\left(k_{v_{11}}\right) & =(11,11,121),
\end{aligned}
$$

and

$$
\operatorname{ind}_{G_{v_{401}}}^{G} \chi_{G_{v_{401}}}\left(k_{v_{401}}\right)=(401,1,401) .
$$

Recall that each element $z \in \zeta(\mathbb{Q}[G])$ is represented by a tuple $z=\left(z_{\chi_{1}}, z_{\chi_{2}}, z_{\chi_{3}}\right)$.
Although we cannot be sure that we have computed the full Mordell-Weil group $E(K)$, it seems most likely that we have found a subgroup of finite index and that the only primes that possibly divide this index are 2 and 5. Therefore, as long as we exclude these primes from our considerations, we still obtain some evidence for the integrality conjecture.

Assuming $E(K)=\left\langle P_{1}, P_{2}\right\rangle$, we derive from the BSD conjecture the conjectural order $\# \amalg(E / K)=5^{8}$. Thus we have $\mathrm{HP}=\{2,5,11,401\}$, and by Corollary 4.8 , the ETNC is numerically confirmed outside HP.

As already mentioned, we cannot expect any integrality statements for $l=2,5$, which would also not be possible for other reasons, because for $l=2$ we cannot perform our computations, because 2 divides $\# G$, and $l=5$ divides $\# G$ and we have nontrivial cohomology modules such as $\amalg(E / K)$ and $E(K)$, which may not be $\mathbb{Z}_{l}[G]$ perfect. Also, Hypothesis 4.1(5) is not satisfied.

For $l \in\{11,401\}$, the group $K_{0}\left(\mathbb{Z}_{l}[G], \mathbb{Q}_{l}\right)_{\text {tors }}$ is trivial, and from the above data, one easily deduces the validity of the $l$-part of the ETNC.

\section{ACKNOWLEDGMENTS}

I am very grateful to David Burns and Tom Fisher for their interest and helpful discussions. I also would like to thank the referee for his careful reading of the manuscript and many valuable comments.

\section{REFERENCES}

[Bley 10] W. Bley. "Numerical Evidence for the Equivariant Birch and Swinnerton-Dyer Conjecture (Part II)." Math. Comp. in press.

[Bley and Burns 03] W. Bley and D. Burns. "Equivariant Epsilon Constants, Discriminants and Étale Cohomology." Proc. London Math. Soc. 87 (2003), 545-590.

[Bley and Johnston 08] W. Bley and H. Johnston. "Computing Generators of Free Modules over Orders in Group Algebras." J. Algebra (Computational Section) 320 (2008), 836-852.

[Bley and Johnston 11] W. Bley and H. Johnston. "Computing Generators of Free Modules over Orders in Group Algebras II." Math. Comp. 80 (2011), 2411-2434.

[Bley and Wilson 09] W. Bley and S. M. J. Wilson. "Computations in Relative Algebraic K-Groups." London Math. Soc. J. Comp. and Math. 12 (2009), 166-194.

[Bloch and Kato 90] S. Bloch and K. Kato. "L-Functions and Tamagawa Numbers of Motives." In The Grothendieck- 
festschrift, vol. 1, Progress in Math. 86, pp. 333-400. Boston: Birkhäuser, 1990.

[Bouganis and Dokchitser 07] T. Bouganis and V. Dokchitser. "Algebraicity of $L$-Values for Elliptic Curves in a False Tate Curve Tower." Math. Proc. Cambridge Philos. Soc. 142 (2007), 193-204.

[Breuning 04] M. Breuning. "On Equivariant Global Epsilon Constants for Certain Dihedral Extensions." Math. Comp. 73 (2004), 881-898.

[Breuning and Burns 05] M. Breuning and D. Burns. "Additivity of Euler Characteristics in Relative Algebraic K-Groups." Homology, Homotopy and Applications 7 (2005), 11-36.

[Breuning and Burns 07] M. Breuning and D. Burns, "Leading Terms of Artin $L$-Functions at $s=0$ and $s=1$." Compositio Math. 143 (2007), 1427-1464.

[Burns 04] D. Burns. "Equivariant Whitehead Torsion and Refined Euler Characteristics." In Number Theory, CRM Proceedings and Lecture Notes 36, pp. 35-59. Providence: American Mathematical Society, 2004.

[Burns 09] D. Burns. "Leading Terms and Values of Emotivic L-Functions." Pure App. Math. Q. 6 (2010) (John Tate Special Issue, Part II), 83-172.

[Burns and Flach 96] D. Burns and M. Flach. "Motivic $L$ Functions and Galois Module Structures." Math. Ann. 305 (1996) 65-102.

[Burns and Flach 01] D. Burns and M. Flach. "Tamagawa Numbers for Motives with (Non-commutative) Coefficients." Documenta Math. 6 (2001), 501-570.

[Curtis and Reiner 87] C. Curtis and I. Reiner. Methods of Representation Theory, vols. I and II. New York: Wiley, 1981 and 1987.

[Darmon 06] H. Darmon. "Heegner Points, Stark-Heegner Points and Values of $L$-Series." In International Congress of Mathematicians, vol. II, pp. 313-345. Zurich: Eur. Math. Soc., 2006.

[Dokchitser 04] T. Dokchitser. "Computing Special Values of Motivic L-Functions." Experiment. Math. 13 (2004), 137149.

[Fearnley and Kisilevsky 10] J. Fearnley and H. Kisilevsky. "Critical Values of Derivatives of Twisted Elliptic $L$ Functions." Experiment. Math. 19 (2010), 149-160.

[Flach 04] M. Flach. "The Equivariant Tamagawa Number Conjecture: A Survey." In Stark's Conjecture: Recent Progress and New Directions, edited by D. Burns, C.
Popescu, J. Sands, and D. Solomon, Contemp. Math. 358, pp. 79-126. Providence: Amer. Math. Soc., 2004.

[Flach 09] M. Flach. "Iwasawa Theory and Motivic $L$ Functions." Pure and Applied Mathematics Quarterly 5:1 (2009), 255-294.

[Fröhlich 89] A. Fröhlich. " $L$-Values at Zero and Multiplicative Galois Module Structure (also Galois-Gauss Sums and Additive Galois Module Structure)." J. Reine Angew. Math 397 (1989) 42-99.

[Gross and Zagier 86] B. H. Gross and D. B. Zagier. "Heegner Points and Derivatives of $L$ Series." Inventiones Mathematicae 84:2 (1986) 225320 .

[Grothendieck 72] A. Grothendieck. Groupes de Monodromie en Géométrie Algébrique (SGA 7 I), Lecture Notes in Math. 288. New York: Springer, 1972.

[Kings 09] G. Kings. "An Introduction to the Equivariant Tamagawa Number Conjecture: The Relation to the Birch-Swinnerton-Dyer Conjecture." Preprint Nr. 26/2009, Universität Regensburg, 2009.

[Kolyvagin 90] V. A. Kolyvagin. "Euler Systems." In The Grothendieck Festschrift, Progr. in Math. 87, pp. 435483. Boston: Birkhäuser, 1990.

[Kolyvagin and Logachev 90] V. A. Kolyvagin and D. Y. Logachev. "Finiteness of the Shafarevich-Tate Group and the Group of Rational Points for Some Modular Abelian Varieties." Leningrad Math. J. 1 (1990), 1229-1253.

[Kolyvagin and Logachev 92] V. A. Kolyvagin and D. Y. Logachev. "Finiteness of Ш over Totally Real Fields." USSR Izvestiya 39 (1992), 829-853.

[Mazur and Tate 87] B. Mazur and J. Tate. "Refined Conjectures of the Birch and Swinnerton-Dyer Type." Duke Math. J. 54 (1987), 711-750.

[Navilarekallu 88] T. Navilarekallu. "Equivariant BirchSwinnerton-Dyer Conjecture for the Base Change of Elliptic Curves: An Example." International Mathematics Research Notices (2008), 33 pages.

[Niziol 93] W. Niziol. "Cohomology of Crystalline Representations." Duke Math. J. 71 (1993), 747-791.

[Shimura 77] G. Shimura. "On the Periods of Modular Forms." Math. Ann. 229 (1977), 211-221.

[Shimura 78] G. Shimura. "The Special Values of the Zeta Functions Associated to Hilbert Modular Forms." Duke Math. J. 45 (1978), 637-679. 
[Silverman 86] J. H. Silverman. The Arithmetic of Elliptic Curves. New York: Springer, 1986.

[Swan 68] R. G. Swan. Algebraic K-Theory, Lecture Notes in Mathematics 76. New York: Springer, 1968.

[Venjakob 07] O. Venjakob. "From the Birch and SwinnertonDyer Conjecture over the Equivariant Tamagawa Num- ber Conjecture to Non-commutative Iwasawa Theory." In L-Functions and Galois Representations, London Math. Soc. Lecture Note Ser. 320, pp. 333-380. Cambridge, UK: Cambridge University Press, 2007.

[Zhang 01] S. Zhang. "Heights of Heegner Points on Shimura Curves." Annals of Math. 153 (2001), 27-147.

Werner Bley, Mathematisches Institut der Universität München, Theresienstr. 39, 80333 München, Germany (bley@math.lmu.de)

Received June 15, 2010; accepted October 8, 2010. 